\title{
Discriminating four tectonic settings: Five new geochemical diagrams for basic and ultrabasic volcanic rocks based on log-ratio transformation of major-element data
}

\author{
Surendra P Verma ${ }^{1, *}$, Mirna Guevara ${ }^{1}$ and Salil Agrawal ${ }^{2}$ \\ ${ }^{1}$ Centro de Investigación en Energía, Universidad Nacional Autónoma de México, Priv. Xochicalco s/no., \\ Col Centro, Apartado Postal 34, Temixco 62580, Mexico. \\ ${ }^{2}$ Department of Geology, University of Rajasthan, Jaipur 302004 , India. \\ *e-mail:spv@cie.unam.mx
}

\begin{abstract}
We present five new discriminant function diagrams based on an extensive database representative of basic and ultrabasic rocks from four tectonic settings of island arc, continental rift, ocean-island, and mid-ocean ridge. These diagrams were obtained after $\log _{\mathrm{e}}$-transformation of concentration ratios of major-elements - a technique recommended for a correct statistical treatment of compositional data. Higher $\%$ success rates (overall values from $\sim 83$ to $97 \%$ ) were obtained for proposing these new diagrams as compared to those ( $\sim 82$ to $94 \%)$ obtained from the discriminant analysis of the raw major-element concentration data (i.e., without the $\log _{\mathrm{e}}$-transformation and without taking ratios of the compositional data, but using exactly the same database to provide an unbiased comparison), suggesting that such a data transformation constitutes a statistically correct and recommended technique. The new diagrams also resulted in less mis-classification of basic and ultrabasic rocks from known tectonic settings than the diagrams obtained from the raw data. The use of these highly successful new discriminant function diagrams is illustrated using Miocene to Recent basic and ultrabasic rocks from three areas of Mexico with complex or controversial tectonic settings (Mexican Volcanic Belt, Los Tuxtlas volcanic field, and Eastern Alkaline Province), as well as older rocks from three areas (Deccan, Malani, and Bastar) of India. Additionally, the major-element data from two 'known' continental arc settings are used to show that they are similar to those from the island arc setting. Continental rift setting is inferred for all Mexican cases and for one cratonic area of India (Malani) and an IAB setting for the Bastar craton. The Deccan flood basalt province of India is used to warn against an indiscriminate use of those discrimination diagrams that do not explicitly include the likely setting of the area under evaluation. An Excel template is also provided for an easy application of these new diagrams for discriminating the four settings considered in this work.
\end{abstract}

\section{Introduction}

Contemporaneously with the development of plate tectonics and the recognition of specific tectonic settings such as island arc, continental rift, oceanisland, and mid-ocean ridge, the idea that magmas from different tectonic settings might be distinguishable in their chemistry was pioneered by Pearce and Cann $(1971,1973)$. Since then, numerous bivariate and ternary tectonomagmatic diagrams have appeared in the literature (for more details see, e.g., Rollinson 1993; Verma 2000a, 2006; Agrawal et al 2004). These diagrams provide additional evidence, complementary to the

Keywords. Log-transformation; compositional space; discriminant function analysis; standard igneous norm and volcanic rock classification system computer program; Mexico; India. 
field evidence, and suggest an affiliation rather than an unequivocal confirmation of the tectonic environment.

From the statistical point of view, probably a major advance in the proposal and functioning of discrimination diagrams came with the introduction of discriminant analysis, in which a large number of variables are examined to isolate those that most effectively classify the samples in their predefined classes or groups. As early as 1965 Chayes and Velde (1965) used discriminant functions to distinguish between circum-oceanic and ocean-island basaltic lavas. Later, Pearce (1976) used 8 major-elements $\left(\mathrm{SiO}_{2}, \mathrm{TiO}_{2}, \mathrm{Al}_{2} \mathrm{O}_{3}, \mathrm{FeO}\right.$, $\mathrm{MgO}, \mathrm{CaO}, \mathrm{Na}_{2} \mathrm{O}$, and $\mathrm{K}_{2} \mathrm{O}$ ) in a total of 358 samples of basaltic rocks and 6 classes to propose new discriminant function diagrams. The linear field boundaries between the groups were, however, drawn by 'eye' (Pearce 1976, p. 22), instead of some objective procedure. Soon afterwards, Yellur and Nair (1978) used Pearce (1976) discriminant function and other diagrams to infer the tectonic environment of Chitradurga metabasalts in south India.

The problem of 'eye-fitted boundaries' persisted in the proposal of most discrimination diagrams until Agrawal (1999) suggested how to replace these eye-fitted boundaries by probability-based surface boundaries. The other problem in the creation of such discrimination diagrams was the inadequacy of the dataset used to represent the population, i.e., the statistical sample probably was not representative of the population. For example, only 358 samples were used by Pearce (1976) to represent 6 classes; many other diagrams are based on similar or still smaller data sets (e.g., 507 samples from 5 settings by Mullen 1983; 35 mean values from about 300 analysis for 5 classes by Butler and Woronow 1986). The lack of representativeness as well as the use of 'eye-fitted' boundaries might be the reasons why Armstrong-Altrin and Verma (2005) found that many tectonic setting discrimination diagrams proposed and used for sedimentary rocks do not work properly.

Both these shortcomings of discrimination diagrams (lack of representativeness of the database and use of 'eye-fitted' boundaries) were recently overcome by Agrawal et al (2004), who used a total of 1159 samples of mostly basic rocks from 4 tectonic settings (island arc, continental rift, oceanisland, and mid-ocean ridge) and performed the discriminant analysis to propose 5 new discriminant function diagrams, in which the boundaries were probability-based surfaces (or lines in twodimensions).

Another problem attacked or addressed by none of the proposals of discrimination diagrams using linear discriminant analysis, as of today, is the 'closure' or 'constant sum' of compositional data including the major-elements in rocks (e.g., Chayes 1960, 1983; Skala 1977, 1979; Aitchison 1986; Reyment 1987; Woronow and Love 1990; Rollinson 1993; Reyment and Savazzi 1999). One of the rare exceptions is Butler and Woronow (1986) who used a small set of $35 \mathrm{TiO}_{2}, \mathrm{Zr}, \mathrm{Y}$, and Sr average analyses (based on about 300 individual analyses) of basalts to perform principal component analysis (but not linear discriminant analysis) of logcentered components.

As is well known, the compositional data ideally sum to 1 (constant sum of parts) or $100 \%$ (the 'closure' problem persists even if the constituents of analysis do not sum exactly to $100 \%$ ). This makes them statistically dependent of each other, for example, if we suppose that the rock composition is represented by 10 major-elements or oxides $\left(\mathrm{SiO}_{2}, \mathrm{TiO}_{2}, \mathrm{Al}_{2} \mathrm{O}_{3}, \mathrm{Fe}_{2} \mathrm{O}_{3}^{\mathrm{t}}, \mathrm{MnO}, \mathrm{MgO}\right.$, $\mathrm{CaO}, \mathrm{Na}_{2} \mathrm{O}, \mathrm{K}_{2} \mathrm{O}$, and $\mathrm{P}_{2} \mathrm{O}_{5}$ ), all parts are mutually dependent because all of them sum up ideally to 1 or $100 \%$. Now, if we assume that all parts are non-zero, and we fix one part (no matter which first part we choose, an essential property of the closure or constant sum is that this part has to be less than the whole, i.e., $<1$ or $<100 \%$ ), the second part (irrespective of which part we imagine to be the second), although not totally predictable, has to be $<(1$-first part) or $<(100 \%$-first part\%) (i.e., partly predefined and dependent). The third and later parts will also be partly defined, but within consecutively smaller ranges. The final (10th) part will be totally predictable, defined, and dependent. These interdependences arising from the closure problem are an undesirable property for any statistical analysis, including the frequently used bivariate 'Harker-type' and ternary diagrams for the interpretation of igneous rock chemistry (for the latter, the situation is even worse because of the renormalization - to 100 - of the three parts used for constructing them; see Butler 1979). According to Aitchison (1986), the study of compositions is essentially concerned with the relative magnitudes of the parts rather than their absolute values, which leads to the conclusion that we should think in terms of ratios (e.g., Rollinson 1993) and perhaps some additional transformation of these ratio data in order to free the sample space. We must, however, be aware of the danger of spurious correlations between certain kind of ratios (e.g., Chayes 1978; Butler 1986).

Our present work is intended to address the issue of closure or constant sum, while complying with the other two aspects - the statistical sample representative of the population and an objective procedure for drawing field boundaries, to arrive at a 


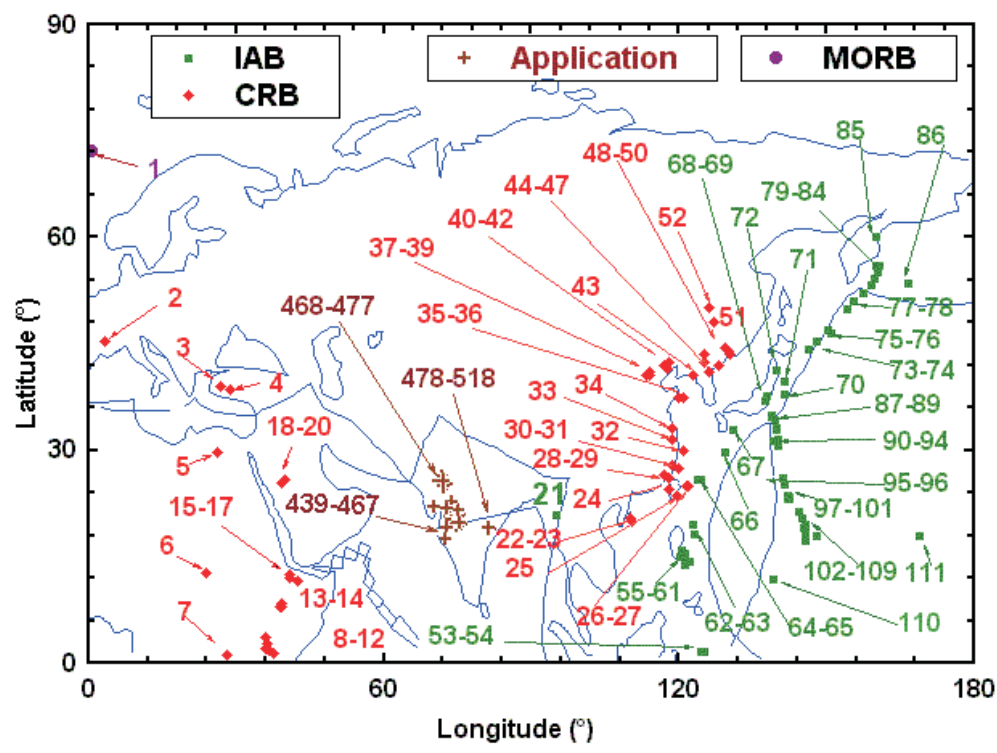

Figure 1. Schematic location of basic (and ultrabasic) rock samples in the quadrant $0-180^{\circ}$ longitude and $0-90^{\circ} \mathrm{N}$ latitude (northern part of the eastern hemisphere: Asia, Europe, and part of Africa). The symbols used are shown as inset. For meaning of \# locations refer to table 1, and for \# application locations, table 9.

statistically sound or valid discriminant analysis of major-element data. We present a set of five, highly successful, new discrimination diagrams (using linear discriminant analysis of $\log _{\mathrm{e}}$-transformation of major-element ratios). We performed discriminant analysis of actual major-element concentration data from the new extended database and compared the success rates with our earlier work with a smaller database, as well as with those obtained from the $\log _{\mathrm{e}}$-transformation of concentration ratios to show that the $\log _{\mathrm{e}}$-transformation is a recommended, statistically-correct procedure for handling compositional data. We also include some specific examples for illustration purposes. Finally, we provide an MS-Excel template to help interested persons in using our new discriminant function diagrams.

\section{Database}

We prepared a representative database of 10 or 11 major-elements (depending on the availability of the total iron as $\mathrm{Fe}_{2} \mathrm{O}_{3}$ and $\mathrm{FeO}$ ) in mostly basic rocks (ultrabasic rocks were included from wherever available) from mainly 4 tectonic settings (figures 1-4; table 1): island arc basic rocks (IAB), continental rift basic rocks (CRB), ocean-island basic rocks (OIB), and mid-ocean ridge basic rocks (MORB). As in Agrawal et al (2004), we note that continental arc is a missing set because the majorelement characteristics of basic rocks from this setting are very similar to those from the island arc; to support this claim, we will use examples from this setting to show that the rocks from continental arc plot mainly in the island arc field.

Our present database contains a total of 2732 samples (table 2) as follows: 463 samples for IAB (named group 1), 771 samples for CRB (group 2), 572 samples for OIB (group 3), and 926 samples for MORB (group 4). The training set consisted of 2332 samples, whereas the testing set was established from randomly drawn 400 samples, 100 from each tectonic setting. The present database contains more samples (2732 versus 1159 samples; table 2) than our earlier study (Agrawal et al 2004).

The random selection of samples for the testing set was carried out by a simple computer program (in QUICKBASIC written by one of us $\mathrm{S}$ Agrawal) for generating random numbers. For example, for the IAB group for which we had 463 samples and we wished to randomly separate 100 samples, 100 random numbers between 1 and 463 (the total number of samples in this group) were generated and the samples corresponding to these random numbers were assigned to the testing group. Similarly, for the CRB group 100 random numbers were generated between 1 and 771 (being the total number of CRB samples in our database) and so on to complete the four settings.

The criteria for choosing the samples in our database were the same as in Agrawal et al (2004), viz., the tectonic setting described explicitly and unambiguously by the author(s), $\left(\mathrm{SiO}_{2}\right)_{\text {adj }}$ content $\leq 52 \%$ (the subscript adj refers to the adjusted data as explained below), and age mostly Piocene to Recent (although some Late Miocene rocks were 


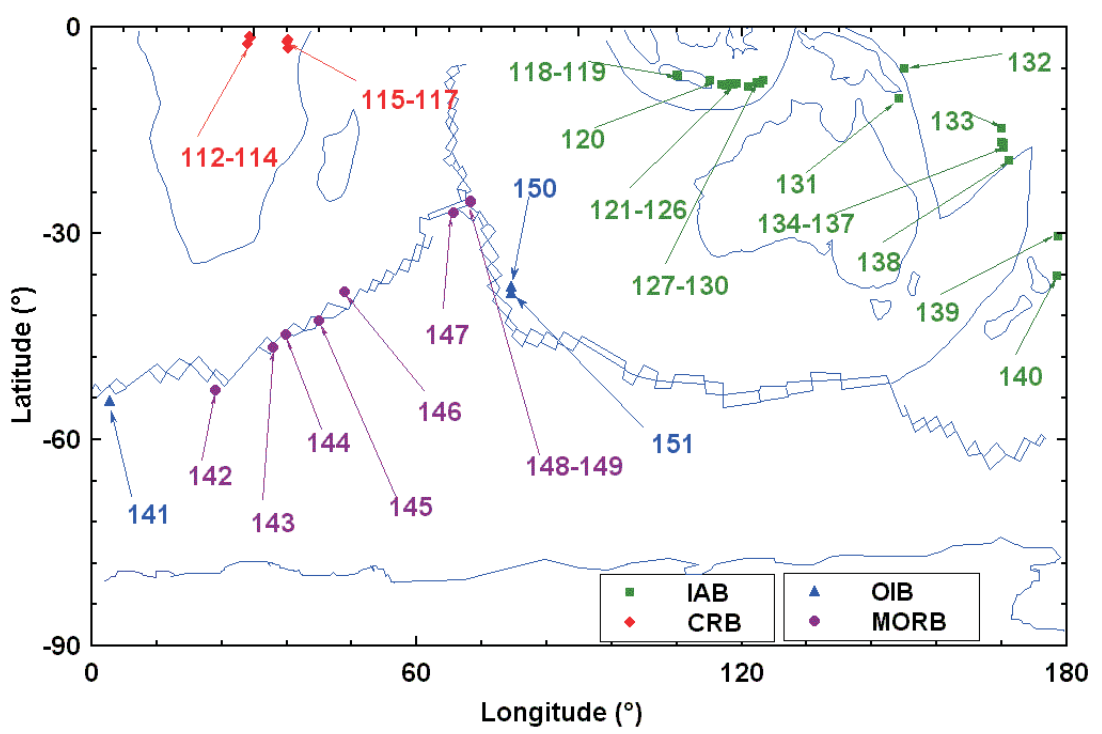

Figure 2. Schematic location of basic (and ultrabasic) rock samples in the quadrant $0-180^{\circ}$ longitude and $0-90^{\circ} \mathrm{S}$ latitude (southern part of the eastern hemisphere: Australia, New Zealand, part of Asia, and southern part of Africa). The symbols used are shown as inset. For meaning of \# locations refer to table 1.

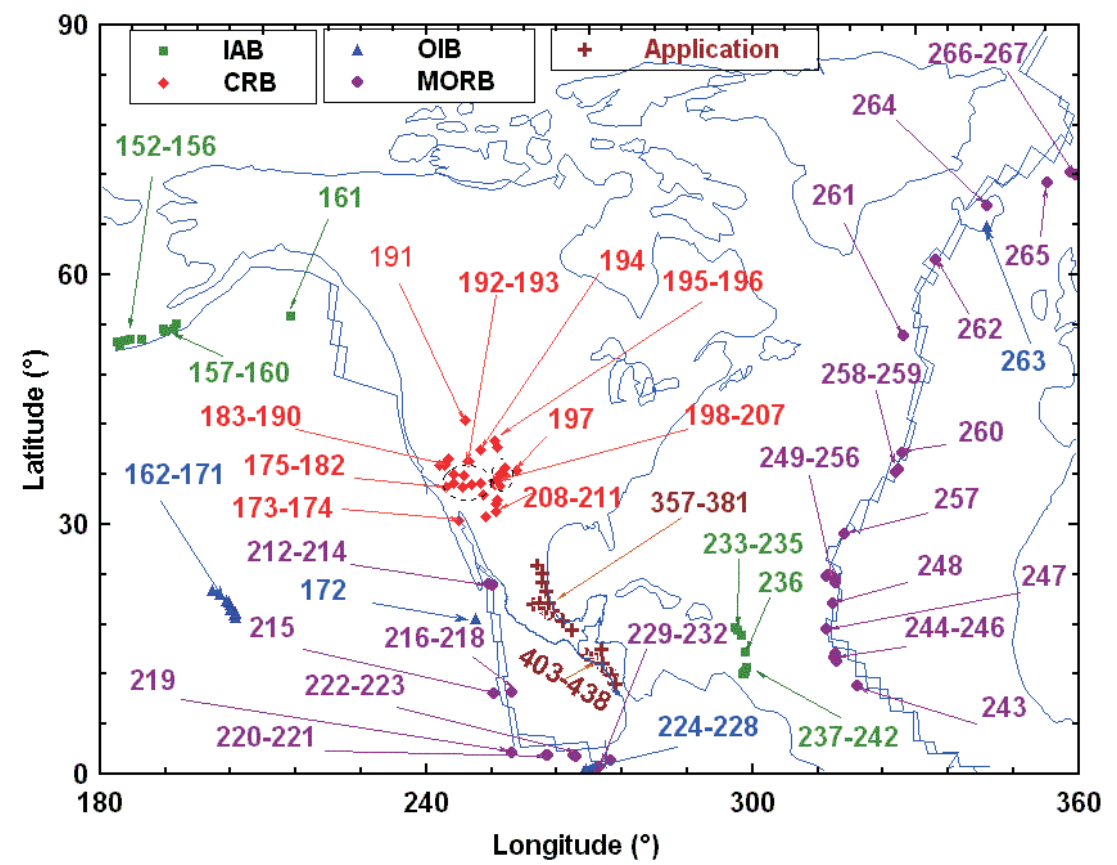

Figure 3. Schematic location of basic (and ultrabasic) rock samples in the quadrant $180-360^{\circ}$ longitude and $0-90^{\circ} \mathrm{N}$ latitude (northern part of the western hemisphere: north America, central America, northern part of south America, and part of Atlantic and Pacific Oceans). The symbols used are shown as inset. For meaning of \# locations refer to table 1, and for \# application locations, table 9.

also included). Inclusion of samples with $\left(\mathrm{SiO}_{2}\right)_{\text {adj }}$ content $\leq 52 \%$ also means that basic as well as ultrabasic rocks from all tectonic settings were present in our database although ultrabasic rocks from the IAB and MORB were less numerous than from the other two settings (CRB and OIB). Further, our experience shows that the inclusion of a few additional samples with $\left(\mathrm{SiO}_{2}\right)_{\text {adj }}$ somewhat higher (up to $\sim 53 \%$ ), along with the basic and ultrabasic samples, provides statistically similar results. Thus, mostly basic and ultrabasic rocks, without the application of any other criteria for primitive mantle-derived magmas (see Velasco-Tapia and Verma 2001 for a synthesis 


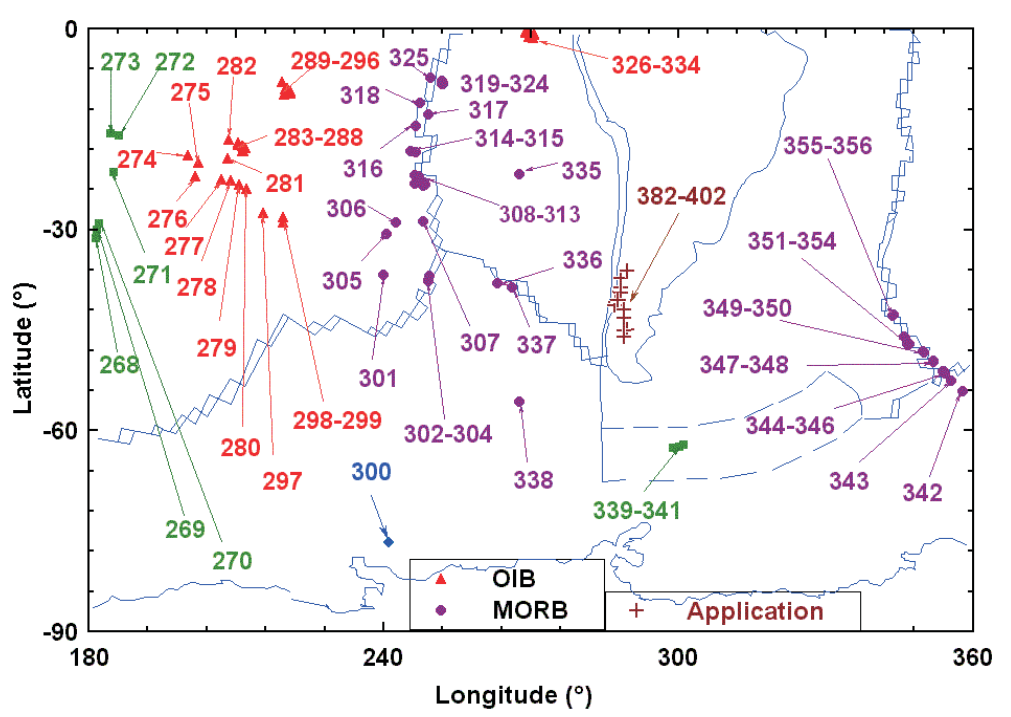

Figure 4. Schematic location of basic rock samples in the quadrant $180-360^{\circ}$ longitude and 0-90 $\mathrm{S}$ latitude (southern part of the western hemisphere: part of south America, Atlantic and Pacific Oceans). The symbols used are shown as inset. For meaning of \# locations refer to table 1, and for \# application locations, table 9.

of such criteria), were compiled for this study. Therefore, effects of magma evolution processes, such as fractional crystallization, magma mixing, or assimilation coupled or uncoupled with fractional crystallization, are also taken into account, at least indirectly, because our database includes all ultrabasic and basic rock samples irrespective of whether they are primitive or evolved rocks in terms of these processes. In summary, the limit of about $52 \%\left(\mathrm{SiO}_{2}\right)_{\text {adj }}$ was chosen simply because we wanted to use only the basic and ultrabasic rocks in this work; our future work will be directed towards handling of more differentiated intermediate and acid igneous rocks.

Specifically, for identifying ultrabasic and basic magmas according to the IUGS Subcommission on the Systematics of Igneous Rocks (Le Bas et al 1986), the volcanic rock classification and TAS diagram should be prepared on an anhydrous 100\% adjusted basis after a proper Fe-adjustment of the major-element data, i.e., using both $\mathrm{Fe}_{2} \mathrm{O}_{3}$ and $\mathrm{FeO}$ varieties and not simply total $\mathrm{Fe}$ as $\mathrm{Fe}_{2} \mathrm{O}_{3}^{\mathrm{t}}$ or $\mathrm{FeO}^{\mathrm{t}}$. The Subcommission recommended that either actually-analyzed $\mathrm{Fe}_{2} \mathrm{O}_{3}$ and $\mathrm{FeO}$ concentrations be used before the adjustment to $100 \%$, or when only total $\mathrm{Fe}$ is available, Le Maitre (1976) method for $\mathrm{Fe}_{2} \mathrm{O}_{3}$ and $\mathrm{FeO}$ calculations should be used. However, the use of actually measured $\mathrm{Fe}_{2} \mathrm{O}_{3}$ and $\mathrm{FeO}$ concentrations in igneous rocks for rock classification may not be appropriate, in spite of the Subcommission's recommendations, because inevitable, extensive post-eruptive changes in their values take place, according to Middlemost (1989) who, in fact, evaluated the different ways for estimating the $\mathrm{Fe}_{2} \mathrm{O}_{3} / \mathrm{FeO}$ ratio and proposed rock-type based values for the division of total $\mathrm{Fe}$ into the $\mathrm{Fe}_{2} \mathrm{O}_{3}$ and $\mathrm{FeO}$ varieties. And this subdivision is a difficult task without using a suitable computer program, such as SINCLAS standard igneous norm and volcanic rock classification system (Verma et al 2002).

All data were, therefore, processed using SINCLAS computer program under the option of Middlemost (1989) for iron-oxidation ratio calculations and $100 \%$ adjustment on an anhydrous basis (Verma et al 2002); this computer program provides highly accurate and consistent results as documented by Verma et al (2003). The data processing by SINCLAS is thus a required step because we wish to separate the total Fe into its two types - the $\mathrm{Fe}_{2} \mathrm{O}_{3}$ and $\mathrm{FeO}$ varieties, and to choose the appropriate samples (with $\left(\mathrm{SiO}_{2}\right)_{\mathrm{adj}}$ content $\leq 52 \%$ ) in our database before the transformation of the data. Even for the samples with $\mathrm{Fe}_{2} \mathrm{O}_{3}$ and $\mathrm{FeO}$ individually reported, we wish to standardize this subdivision of iron according to some standard recommendations (e.g., Middlemost 1989) by first combining these parts into total $\mathrm{Fe}_{2} \mathrm{O}_{3}^{\mathrm{t}}$ and then subdividing it into $\mathrm{Fe}_{2} \mathrm{O}_{3}$ and $\mathrm{FeO}$ varieties. This is done because all data should be handled in exactly the same way irrespective of whether iron was reported in the literature as total $\mathrm{Fe}_{2} \mathrm{O}_{3}^{\mathrm{t}}$ or total $\mathrm{FeO}^{\mathrm{t}}$ or as separate varieties. Thus, we note that the data processing using SINCLAS will be mandatory for all applications because, in order to be consistent with the procedure of these new discriminant function diagrams, all samples must be processed for the calculation of $\mathrm{Fe}_{2} \mathrm{O}_{3}$ and $\mathrm{FeO}$ contents, according to the proposal of Middlemost (1989), which in 
Table 1. Sample locations for the database construction (testing and training sets).

\begin{tabular}{|c|c|c|c|c|c|c|}
\hline $\begin{array}{l}\text { Site } \\
\#\end{array}$ & $\underset{\#}{\text { Figure }}$ & $\begin{array}{l}\text { Longitude } \\
\qquad\left(^{\circ}\right)\end{array}$ & $\begin{array}{l}\text { Latitude } \\
\quad\left({ }^{\circ}\right)\end{array}$ & Country/Province & $\begin{array}{c}\text { No. of } \\
\text { samples }\end{array}$ & Reference \\
\hline \multicolumn{7}{|c|}{ Island arc } \\
\hline 21 & 1 & 95 & 20.8 & Burma Arc (Mt. Popa volcano) & 4 & Stephenson and Marshall (1984) \\
\hline 53 & 1 & 124.5 & 1.6 & Sangihe Arc (Manado Tua) & 7 & Tatsumi et al (1991) \\
\hline 54 & 1 & 125.18 & 1.5 & Sangihe Arc (Tongkoko) & 1 & Tatsumi et al (1991) \\
\hline 55 & 1 & 121.05 & 13.81 & Luzon Arc & 2 & Miklius et al (1991) \\
\hline 56 & 1 & 121.2 & 14.3 & Philippines Arc (Macolod corridor) & 8 & Knittel et al (1997) \\
\hline 57 & 1 & 122.3 & 14.3 & Philippines Arc (Mt. Arayat) & 16 & Bau and Knittel (1993) \\
\hline 58 & 1 & 122.35 & 14.3 & Philippines Arc (Mt. San Cristobal) & 1 & Defant et al (1989) \\
\hline 59 & 1 & 120.16 & 15.0 & $\begin{array}{l}\text { Luzon Arc (Bataan arc-front, } \\
\text { Mt. Native, Mt. Limay) }\end{array}$ & 2 & Defant et al (1991) \\
\hline 60 & 1 & 120.48 & 15.8 & $\begin{array}{l}\text { Luzon Arc (Bataan arc-front, } \\
\text { Mt. Amorong, Mt. Arayat) }\end{array}$ & 2 & Defant et al (1991) \\
\hline 61 & 1 & 121.35 & 15.5 & Philippines Arc (Mt. Mariveles) & 1 & Defant et al (1989) \\
\hline 62 & 1 & 123.1 & 18.2 & Philippines Arc (Cagua) & 1 & Defant et al (1989) \\
\hline 63 & 1 & 122.9 & 19.5 & Philippines Arc (Babuyan de Claro) & 1 & Defant et al (1989) \\
\hline 64 & 1 & 123.68 & 25.93 & Ryukyu Arc (Kobi-syo) & 3 & Shinjo (1998) \\
\hline 65 & 1 & 124.56 & 25.92 & Ryukyu Arc (Sekibi-syo) & 4 & Shinjo (1998) \\
\hline 66 & 1 & 129.52 & 29.6 & Ryukyu Arc & 5 & Shinjo et al (2000) \\
\hline 67 & 1 & 131.1 & 32.85 & Japan Arc (Kyushu) & 1 & Kita et al (2001) \\
\hline 68 & 1 & 137.5 & 37.05 & Japan Arc (Ueno) & 16 & Kimura et al (2002) \\
\hline 69 & 1 & 138 & 37.57 & Central Japan & 5 & Kimura et al (2002) \\
\hline 70 & 1 & 141.4 & 37.9 & Japan Arc (Zao) & 1 & Sakuyama and Nesbitt (1986) \\
\hline 71 & 1 & 141.6 & 39.6 & Japan Arc (Iwate, Kayo) & 2 & Sakuyama and Nesbitt (1986) \\
\hline 71 & 1 & 141.6 & 39.6 & Japan Arc NE (Iwate) & 2 & Togashi et al (1992) \\
\hline 72 & 1 & 139.8 & 41.35 & Japan Arc (Oshima-Oshima) & 1 & Sakuyama and Nesbitt (1986) \\
\hline 73 & 1 & 146.25 & 44.25 & Kuril Arc (Tyatya volcano) & 9 & Nakagawa et al (2002) \\
\hline 74 & 1 & 147.86 & 45.25 & $\begin{array}{l}\text { Kurile Island Arc (Iturup island, } \\
\text { Demon and Atsonupuri vol.) }\end{array}$ & 2 & Zhuravlev et al (1987) \\
\hline 75 & 1 & 150.9 & 46.37 & $\begin{array}{l}\text { Kurile Island Arc (Gorshkov } \\
\text { volcano) }\end{array}$ & 1 & Zhuravlev et al (1987) \\
\hline 76 & 1 & 150.4 & 46.87 & Kurile Island Arc (Vavilov volcano) & 1 & Zhuravlev et al (1987) \\
\hline 77 & 1 & 154.13 & 49.93 & $\begin{array}{l}\text { Kurile Island Arc (Baliankin } \\
\text { volcano) }\end{array}$ & 1 & Zhuravlev et al (1987) \\
\hline 78 & 1 & 155.43 & 50.93 & Kurile Island Arc (Alaid volcano) & 1 & Zhuravlev et al (1987) \\
\hline 79 & 1 & 157.52 & 52.09 & $\begin{array}{l}\text { Kamchatka Arc (Mutnovsky, } \\
\text { Ksudach) }\end{array}$ & 2 & Kepezhinskas et al (1997) \\
\hline 80 & 1 & 159 & 53.2 & Kamchatka Arc (Avachinsky) & 1 & Kepezhinskas et al (1997) \\
\hline 81 & 1 & 159.88 & 54.12 & Kamchatka Arc (Semyachik) & 1 & Kepezhinskas et al (1997) \\
\hline 82 & 1 & 160.53 & 55.17 & Kamchatka Arc (Nikolka) & 1 & Kepezhinskas et al (1997) \\
\hline 83 & 1 & 160.2 & 55.9 & $\begin{array}{l}\text { Kamchatka Arc (Tolbachik, } \\
\text { Kamen, Ploskiy) }\end{array}$ & 5 & Kepezhinskas et al (1997) \\
\hline 83 & 1 & 160.2 & 55.9 & Kamchatka Arc (Tolbachik) & 1 & Ishikawa et al (2001) \\
\hline 84 & 1 & 160.6 & 56.1 & Kamchatka Arc (Klyuchevskoy) & 2 & Ishikawa et al (2001) \\
\hline 85 & 1 & 160 & 61 & $\begin{array}{l}\text { Kamchatka Arc (Valovayam, } \\
\text { Belaya); approx. Coordinates }\end{array}$ & 4 & Kepezhinskas et al (1997) \\
\hline 86 & 1 & 166.5 & 53.5 & Aleutian Arc (Makushin) & 1 & Kay and Kay (1994) \\
\hline 87 & 1 & 138.8 & 34.9 & Japan Arc (Shirahama) & 2 & Tamura (1994) \\
\hline 88 & 1 & 138.95 & 34.71 & Izu-Bonin Arc (Izu peninsula) & 5 & Tatsumi et al (1992) \\
\hline 89 & 1 & 139.35 & 34.35 & $\begin{array}{l}\text { Izu-Bonin Arc, Japan (Oshima, } \\
\text { Toshima, Miyakejima) }\end{array}$ & 5 & Taylor and Nesbitt (1998) \\
\hline 90 & 1 & 139.7 & 33.2 & Izu-Bonin Arc & 2 & Tatsumi et al (1992) \\
\hline
\end{tabular}


Table 1. (Continued).

\begin{tabular}{|c|c|c|c|c|c|c|}
\hline $\begin{array}{l}\text { Site } \\
\#\end{array}$ & $\underset{\#}{\text { Figure }}$ & $\begin{array}{l}\text { Longitude } \\
\qquad\left(^{\circ}\right)\end{array}$ & $\begin{array}{l}\text { Latitude } \\
\quad\left({ }^{\circ}\right)\end{array}$ & Country/Province & $\begin{array}{c}\text { No. of } \\
\text { samples }\end{array}$ & Reference \\
\hline 91 & 1 & 139.8 & 32.8 & $\begin{array}{l}\text { Izu-Bonin Arc, Japan } \\
\text { (Hachijojima, Aogashima) }\end{array}$ & 8 & Taylor and Nesbitt (1998) \\
\hline 92 & 1 & 140.11 & 31.41 & Izu-Bonin Arc & 1 & Tatsumi et al (1992) \\
\hline 93 & 1 & 139.13 & 31.22 & Izu-Bonin Arc & 1 & Tatsumi et al (1992) \\
\hline 94 & 1 & 140.3 & 30.5 & Izu-Bonin Arc, Japan (Torishima) & 5 & Taylor and Nesbitt (1998) \\
\hline 95 & 1 & 141 & 26.08 & Mariana Arc & 1 & Bloomer et al (1989) \\
\hline 96 & 1 & 141.44 & 25.1 & Mariana Arc & 4 & Bloomer et al (1989) \\
\hline 97 & 1 & 142.12 & 23.55 & Mariana Arc & 3 & Bloomer et al (1989) \\
\hline 98 & 1 & 142.25 & 23.37 & Mariana Arc & 2 & Bloomer et al (1989) \\
\hline 99 & 1 & 142.44 & 23.3 & Mariana Arc & 1 & Bloomer et al (1989) \\
\hline 100 & 1 & 142.44 & 23.18 & Mariana Arc & 1 & Bloomer et al (1989) \\
\hline 101 & 1 & 142.25 & 23.08 & Mariana Arc & 4 & Bloomer et al (1989) \\
\hline 102 & 1 & 144.25 & 21.42 & Mariana Arc & 2 & Bloomer et al (1989) \\
\hline 103 & 1 & 145 & 20.42 & Mariana Arc & 1 & Bloomer et al (1989) \\
\hline 104 & 1 & 145.56 & 19.42 & Mariana Arc & 1 & Bloomer et al (1989) \\
\hline 105 & 1 & 145.25 & 19 & Mariana Arc & 1 & Hole et al (1984) \\
\hline 106 & 1 & 145.65 & 18.75 & Mariana Arc (Agrigan) & 3 & Elliott et al (1997) \\
\hline 107 & 1 & 145.8 & 18.16 & Mariana Arc (Pagan) & 2 & Elliott et al (1997) \\
\hline 108 & 1 & 145.85 & 17.33 & Mariana Arc (Guguam) & 1 & Elliott et al (1997) \\
\hline 109 & 1 & 148 & 18 & Mariana Arc (Northern Islands) & 4 & Woodhead (1988) \\
\hline 110 & 1 & 139.12 & 11.75 & Yap Arc System & 1 & Ohara et al (2002) \\
\hline 111 & 1 & 169 & 18 & $\begin{array}{l}\text { New Hebrides Arc (Urepara-Hunter } \\
\text { Island) }\end{array}$ & 16 & Monzier et al (1997) \\
\hline 118 & 2 & 108 & -7 & Sunda Arc (Java) & 3 & Whitford et al (1979) \\
\hline 119 & 2 & 108.2 & -7.16 & Sunda Arc (Gulunggung) & 2 & Turner and Foden (2001) \\
\hline 120 & 2 & 114.25 & -7.8 & Java Arc (Ringgit Beser) & 2 & Edwards et al (1994) \\
\hline 121 & 2 & 116.4 & -8.45 & Sunda Arc (Rindjani) & 2 & Foden and Varne (1980) \\
\hline 122 & 2 & 117.15 & -8.58 & Sunda Arc (Gunung Sangenges) & 1 & Foden and Varne (1980) \\
\hline 123 & 2 & 118 & -8.25 & Sunda Arc (Tambora) & 3 & Foden and Varne (1980) \\
\hline 124 & 2 & 118.59 & -8.45 & Sunda Banda Arc (Soromundi) & 3 & Foden and Varne (1980) \\
\hline 125 & 2 & 119.08 & -8.2 & Sunda Arc (Sangeang Api) & 2 & Foden and Varne (1980) \\
\hline 126 & 2 & 119.1 & -8.2 & Sunda Arc (Sangeang) & 6 & Turner et al (2003) \\
\hline 127 & 2 & 121.3 & -8.66 & Sunda Arc (Inerie, Ija) & 2 & Stolz et al (1990) \\
\hline 127 & 2 & 121.3 & -8.66 & Sunda Banda Arc (Inerie) & 1 & Wheller et al (1987) \\
\hline 128 & 2 & 122.85 & -8 & Sunda Arc (Mandiri, Boleng) & 2 & Stolz et al (1990) \\
\hline 128 & 2 & 122.85 & -8 & Sunda Banda Arc (Boleng) & 1 & Wheller et al (1987) \\
\hline 129 & 2 & 123.4 & -8.3 & Sunda Arc (Werung) & 2 & Hoogewerff et al (1997) \\
\hline 130 & 2 & 123.94 & -7.7 & Sunda Arc (Batu Tara) & 5 & Stolz et al (1988) \\
\hline 130 & 2 & 123.94 & -7.7 & Sunda Banda Arc (Batu Tara) & 2 & Wheller et al (1987) \\
\hline 131 & 2 & 149 & -10.43 & Papua New Guinea Arc & 4 & Hegner and Smith (1992) \\
\hline 132 & 2 & 150 & -6 & $\begin{array}{l}\text { New British Island Arc (Bangun, } \\
\text { Kimbe, Unea, Undaka) }\end{array}$ & 4 & Woodhead and Johnson (1993) \\
\hline 133 & 2 & 168 & -14.7 & Vanuatu Arc (Mereleva volcano) & 7 & Barsdell (1988) \\
\hline 134 & 2 & 168 & -16.7 & Vanuatu Arc (Epi) & 15 & Barsdell and Berry (1990) \\
\hline 135 & 2 & 168.3 & -17 & New Hebrides Arc (Epi, Efate) & 15 & Dupuy et al (1982) \\
\hline 136 & 2 & 168.43 & -17.57 & $\begin{array}{l}\text { Vanuatu Arc (Efate Island, Quoin } \\
\text { hill) }\end{array}$ & 1 & Raos and Crawford (2004) \\
\hline 137 & 2 & 168.49 & -17.5 & Vanuatu Arc (Efate Island, Emau) & 1 & Raos and Crawford (2004) \\
\hline 138 & 2 & 169.4 & -19.4 & $\begin{array}{l}\text { New Hebrides Arc (Erromango, } \\
\text { Tanna, Aneityum) }\end{array}$ & 11 & Dupuy et al (1982) \\
\hline
\end{tabular}


Table 1. (Continued).

\begin{tabular}{|c|c|c|c|c|c|c|}
\hline $\begin{array}{l}\text { Site } \\
\#\end{array}$ & $\begin{array}{l}\text { Figure } \\
\#\end{array}$ & $\begin{array}{l}\text { Longitude } \\
\quad\left({ }^{\circ}\right)\end{array}$ & $\begin{array}{l}\text { Latitude } \\
\left({ }^{\circ}\right)\end{array}$ & Country/Province & $\begin{array}{c}\text { No. of } \\
\text { samples }\end{array}$ & Reference \\
\hline 139 & 2 & 178.34 & -36.17 & Kermadec Arc (Rumble IV) & 5 & Gamble et al (1995) \\
\hline 140 & 2 & 178.43 & -30.51 & Kermadec Arc (Macauley Island) & 5 & Smith et al (2003) \\
\hline 152 & 3 & 183 & 51.95 & Aleutian Arc (Kanaga, Adagdak) & 2 & Kay and Kay (1994) \\
\hline 153 & 3 & 183.5 & 51.5 & $\begin{array}{l}\text { Aleutian Arc (Finger bay, Hidden } \\
\text { bay) }\end{array}$ & 2 & Kay et al (1982) \\
\hline 154 & 3 & 184.5 & 52.13 & Aleutian Arc (Kasatochi) & 1 & Kay and Kay (1994) \\
\hline 155 & 3 & 185.5 & 52.3 & $\begin{array}{l}\text { Aleutian Arc (Atka, Mount } \\
\text { Kliuchef, and Korovin volcanoes) }\end{array}$ & 7 & Myers et al (2002) \\
\hline 155 & 3 & 185.5 & 52.3 & Aleutian Arc (Adka) & 4 & Myers et al (1985) \\
\hline 156 & 3 & 187.47 & 52.25 & Aleutian Arc center (Turf point) & 1 & Singer et al (1992a) \\
\hline 156 & 3 & 187.47 & 52.25 & Aleutian Arc center (Turf point) & 7 & Singer et al (1992b) \\
\hline 157 & 3 & 191.6 & 53.5 & Aleutian Arc (Okmok volcano) & 6 & Singer et al (1992b) \\
\hline 158 & 3 & 191.8 & 53.2 & Aleutian Arc (Okmok) & 2 & Kay and Kay (1994) \\
\hline 159 & 3 & 193.5 & 53.5 & Aleutian Arc (Captains Bay) & 1 & Nye and Reid (1986) \\
\hline 160 & 3 & 194.08 & 54.08 & Aleutian Arc & 8 & Romick et al (1990) \\
\hline 161 & 3 & 215 & 55 & Aleutian Arc (Cold Bay) & 3 & Brophy (1986) \\
\hline 233 & 3 & 296.77 & 17.63 & Lesser Antilles Arc (Saba Island) & 5 & Defant et al (2001) \\
\hline 234 & 3 & 297.29 & 17.25 & Lesser Antilles Arc (St. Kitts) & 2 & Brown et al (1977) \\
\hline 235 & 3 & 297.82 & 16.69 & $\begin{array}{l}\text { Lesser Antilles Arc (Montserrat, } \\
\text { Soufrière hills) }\end{array}$ & 8 & Zellmer et al (2003) \\
\hline 236 & 3 & 298.71 & 14.66 & Lesser Antilles Arc (Dominica) & 2 & Brown et al (1977) \\
\hline 236 & 3 & 298.82 & 12.86 & Lesser Antilles Arc (Bequia) & 1 & Brown et al (1977) \\
\hline 237 & 3 & 298.82 & 12.86 & Lesser Antilles Arc (Bequia) & 11 & Smith et al (1996) \\
\hline 238 & 3 & 298.6 & 12.35 & Lesser Antilles Arc (Carriacou) & 1 & Brown et al (1977) \\
\hline 239 & 3 & 298.36 & 12.16 & $\begin{array}{l}\text { Lesser Antilles Arc (Pilot Hill, } \\
\text { Montreuil State) }\end{array}$ & 2 & Devine (1995) \\
\hline 240 & 3 & 298.39 & 12.12 & Lesser Antilles Arc (Grenada) & 13 & Thirlwall and Graham (1984) \\
\hline 240 & 3 & 298.39 & 12.12 & Lesser Antilles Arc (Grenada) & 2 & Brown et al (1977) \\
\hline 240 & 3 & 298.39 & 12.12 & Lesser Antilles Arc (Grenada) & 3 & Arculus (1976) \\
\hline 240 & 3 & 298.39 & 12.12 & Lesser Antilles Arc (Grenada) & 29 & Thirlwall et al (1997) \\
\hline 241 & 3 & 298.29 & 12.11 & Lesser Antilles (Grenada)* & 5 & Shimizu and Arculus (1975) \\
\hline 242 & 3 & 298.25 & 12.06 & $\begin{array}{l}\text { Lesser Antilles Arc (Queen's Park, } \\
\text { Radix) }\end{array}$ & 2 & Devine (1995) \\
\hline 268 & 4 & 181.4 & -31.35 & $\begin{array}{l}\text { Tonga Kermadec Arc } \\
\text { (L'Esperance) }\end{array}$ & 2 & Ewart et al (1977) \\
\hline 269 & 4 & 181.8 & -30 & $\begin{array}{l}\text { Tonga Kermadec Arc (Macauley } \\
\text { Island) }\end{array}$ & 8 & Ewart et al (1977) \\
\hline 270 & 4 & 182.19 & -29.06 & $\begin{array}{l}\text { Tonga Kermadec Arc (Raoul island, } \\
\text { Dayrell) }\end{array}$ & 6 & Ewart et al (1977) \\
\hline 271 & 4 & 185.07 & -21.4 & Tonga Kermadec Arc (Eua) & 2 & Bryan et al (1972) \\
\hline 272 & 4 & 186.25 & -16.03 & Tonga Kermadec Arc (Tafahi) & 1 & Ewart et al (1977) \\
\hline 273 & 4 & 184.38 & -15.59 & Tonga Kermadec Arc (Niua Fo'ou) & 1 & Bryan et al (1972) \\
\hline 274 & 4 & 184.38 & -15.59 & Tonga Kermadec Arc (Niua Fo'ou) & 5 & Ewart et al (1977) \\
\hline 339 & 4 & 298.9 & -62.66 & $\begin{array}{l}\text { South Shetland Arc (Byers } \\
\text { Peninsula) }\end{array}$ & 1 & Smellie (1983) \\
\hline 340 & 4 & 300.21 & -62.39 & $\begin{array}{l}\text { South Shetland Arc (Copermine } \\
\text { Peninsula) }\end{array}$ & 1 & Smellie (1983) \\
\hline 341 & 4 & 301 & -62.14 & $\begin{array}{l}\text { South Shetland Arc (Fildes } \\
\text { Peninsula) }\end{array}$ & 1 & Smellie (1983) \\
\hline \multicolumn{7}{|c|}{ Continental rift } \\
\hline 2 & 1 & 3.35 & 45.25 & Massif Central & 21 & Chauvel and Jahn (1984) \\
\hline 2 & 1 & 3.35 & 45.25 & Massif Central & 3 & $\begin{array}{l}\text { Fontaine-Vive and } \\
\text { De Goer De Herve (1984) }\end{array}$ \\
\hline
\end{tabular}


Table 1. (Continued).

\begin{tabular}{|c|c|c|c|c|c|c|}
\hline $\begin{array}{l}\text { Site } \\
\#\end{array}$ & $\begin{array}{l}\text { Figure } \\
\quad \#\end{array}$ & $\begin{array}{l}\text { Longitude } \\
\left(^{\circ}\right)\end{array}$ & $\begin{array}{l}\text { Latitude } \\
\quad\left({ }^{\circ}\right)\end{array}$ & Country/Province & $\begin{array}{c}\text { No. of } \\
\text { samples }\end{array}$ & Reference \\
\hline 3 & 1 & 26.9 & 39 & Western Anatolia (DAB Area) & 4 & Aldanmaz et al (2000) \\
\hline 4 & 1 & 28.7 & 38.54 & Anatolian Graben & 5 & Alici et al (2002) \\
\hline 5 & 1 & 26.2 & 29.6 & Western Anatolia (EGA Area) & 9 & Aldanmaz et al (2000) \\
\hline 6 & 1 & 24 & 12.6 & $\begin{array}{l}\text { Triple Junction, Sudan (Jebel } \\
\text { Marra volcano) }\end{array}$ & 9 & Davidson and Wilson (1989) \\
\hline 7 & 1 & 28.1 & 1.25 & $\begin{array}{l}\text { Gregory Rift, Kenya (Silali } \\
\text { Volcano) }\end{array}$ & 9 & Macdonald et al (1995) \\
\hline 8 & 1 & 35.9 & 3.7 & $\begin{array}{l}\text { Kenya, Turkana Rift (Bird Nest, } \\
\text { Central) }\end{array}$ & 3 & Furman et al (2004) \\
\hline 9 & 1 & 36 & 2 & Kenya Rift (Nathelat) & 1 & Macdonald et al (2001) \\
\hline 10 & 1 & 36.4 & 2.7 & $\begin{array}{l}\text { Kenya, Turkana Rift (Barrier, } \\
\text { South) }\end{array}$ & 2 & Furman et al (2004) \\
\hline 11 & 1 & 36.52 & 1.75 & Kenya Rift (Samburu Hills) & 5 & Kabeto et al (2001) \\
\hline 12 & 1 & 37.5 & 1.3 & Huri Hills, Kenya & 36 & Class et al (1994) \\
\hline 13 & 1 & 39 & 8 & Ethiopian Rift & 14 & Hart et al (1989) \\
\hline 14 & 1 & 39.15 & 8 & Ethiopian Rift & 4 & Trua et al (1999) \\
\hline 15 & 1 & 39.2 & 8.4 & Ethiopian Rift (Gedemsa Volcano) & 4 & Peccerillo et al (2003) \\
\hline 16 & 1 & 41 & 12 & $\begin{array}{l}\text { Triple Junction, Afar, Ethiopia } \\
\text { (M Hararo) }\end{array}$ & 14 & Barrat et al (2003) \\
\hline 17 & 1 & 41 & 12.5 & Afar Rift (Boina Centre) & 6 & Barberi et al (1975) \\
\hline 18 & 1 & 42.5 & 11.5 & Triple Junction, Afar (Dijibouti) & 44 & Deniel et al (1994) \\
\hline 19 & 1 & 39.1 & 25.5 & Saudi Arabia (Harrat Kura) & 3 & Camp et al (1991) \\
\hline 20 & 1 & 40 & 25.8 & $\begin{array}{l}\text { Saudi Arabia (Khaybar and } \\
\text { Ithnayn) }\end{array}$ & 10 & Camp et al (1991) \\
\hline 22 & 1 & 110 & 20.5 & SE China (Haikang-HainanIs) & 3 & Liu et al (1994) \\
\hline 23 & 1 & 110.5 & 20 & SE China (Hainan Island) & 2 & Fan and Hooper (1991) \\
\hline 24 & 1 & 118 & 24.5 & E China (Longhai) & 2 & Fan and Hooper (1991) \\
\hline 24 & 1 & 118 & 24.5 & SE China (Niutoushan) & 8 & Zou et al (2000) \\
\hline 25 & 1 & 119.5 & 23.6 & Taiwan Strait (Penghu Islands) & 1 & Chung et al (1994) \\
\hline 25 & 1 & 119.5 & 23.6 & China (Penghu Island) & 1 & Chung et al (1994) \\
\hline 26 & 1 & 121.5 & 24.9 & Taiwan Strait (NW Taiwan) & 1 & Chung et al (1994) \\
\hline 27 & 1 & 122 & 25 & Taiwan (Kungkuan) & 8 & Chung et al (1995) \\
\hline 28 & 1 & 118 & 26 & Taiwan Strait (Mt. Fujian) & 3 & Chung et al (1994) \\
\hline 28 & 1 & 118 & 26 & China (Fujian) & 1 & Chung et al (1994) \\
\hline 29 & 1 & 117 & 26.5 & E China (Mingxi) & 4 & Zou et al (2000) \\
\hline 29 & 1 & 117 & 26.5 & E China (Mingxi) & 1 & Liu et al (1994) \\
\hline 29 & 1 & 117 & 26.5 & E China (Minxi) & 1 & Fan and Hooper (1991) \\
\hline 30 & 1 & 118.5 & 28 & E China (Songxi) & 2 & Peng et al (1986) \\
\hline 30 & 1 & 118.5 & 28 & E China (Longyou-Xilong) & 4 & Zou et al (2000) \\
\hline 31 & 1 & 120 & 27.5 & E China (Minquing) & 1 & Fan and Hooper (1991) \\
\hline 32 & 1 & 121 & 30 & E China (Xinchang) & 2 & Fan and Hooper (1991) \\
\hline 33 & 1 & 118.5 & 31.5 & E China (Anhui-Jiangsu) & 2 & Peng et al (1986) \\
\hline 34 & 1 & 118.5 & 33 & E China (Nushan-Fangshan) & 3 & Liu et al (1994) \\
\hline 34 & 1 & 118.5 & 33 & E China (Jiashan-Xuyi-Liuhe) & 4 & Fan and Hooper (1991) \\
\hline 34 & 1 & 118.5 & 33 & $\begin{array}{l}\text { E China (Nushan-Tashan- } \\
\text { Fangshan) }\end{array}$ & 10 & Zou et al (2000) \\
\hline 35 & 1 & 120 & 37.5 & E China (Shandong) & 10 & Peng et al (1986) \\
\hline 36 & 1 & 121 & 37.5 & E China (Xixia) & 1 & Liu et al (1994) \\
\hline 36 & 1 & 121 & 37.5 & $\begin{array}{l}\text { E China (Penlai-Qixia-Wudi- } \\
\text { Linqu) }\end{array}$ & 5 & Fan and Hooper (1991) \\
\hline 37 & 1 & 113.5 & 40.5 & E China (Fanzhi-Datong-Jining) & 5 & Fan and Hooper (1991) \\
\hline
\end{tabular}


Table 1. (Continued).

\begin{tabular}{|c|c|c|c|c|c|c|}
\hline $\begin{array}{l}\text { Site } \\
\#\end{array}$ & $\underset{\#}{\text { Figure }}$ & $\begin{array}{l}\text { Longitude } \\
\qquad\left(^{\circ}\right)\end{array}$ & $\begin{array}{l}\text { Latitude } \\
\quad\left({ }^{\circ}\right)\end{array}$ & Country/Province & $\begin{array}{c}\text { No. of } \\
\text { samples }\end{array}$ & Reference \\
\hline 38 & 1 & 114 & 40.5 & E China (Hanobar-Datong) & 6 & Basu et al (1991) \\
\hline 39 & 1 & 114 & 41 & E China (Hannuoba) & 67 & Zhi et al (1990) \\
\hline 39 & 1 & 114 & 41 & E China (Hannuoba) & 3 & Liu et al (1994) \\
\hline 39 & 1 & 114 & 41 & E China (Hannuoba) & 2 & Fan and Hooper (1991) \\
\hline 40 & 1 & 117.5 & 41.5 & E China (Pinquan) & 3 & Fan and Hooper (1991) \\
\hline 41 & 1 & 117 & 42 & E China (Weichang) & 4 & Fan and Hooper (1991) \\
\hline 42 & 1 & 118 & 42.5 & N China (Chifeng) & 8 & Han et al (1999) \\
\hline 43 & 1 & 123 & 40.5 & E China (Liaoning) & 2 & Peng et al (1986) \\
\hline 44 & 1 & 126 & 41 & E China (Kuandian) & 5 & Liu et al (1994) \\
\hline 44 & 1 & 126 & 41 & E China (Kuandian) & 1 & Fan and Hooper (1991) \\
\hline 45 & 1 & 125 & 42.5 & $\begin{array}{l}\text { NE China (Huinan-Dadung- } \\
\text { Fushun-Qingyan) }\end{array}$ & 7 & Liu et al (1994) \\
\hline 46 & 1 & 125 & 43.5 & E China (Jilin) & 1 & Peng et al (1986) \\
\hline 46 & 1 & 125 & 43.5 & $\begin{array}{l}\text { NE China (Changchuen-Shuanlia- } \\
\text { Yitong) }\end{array}$ & 5 & Fan and Hooper (1991) \\
\hline 47 & 1 & 128 & 42 & NE China (Kirin) & 13 & Hsu et al (2000) \\
\hline 48 & 1 & 130.5 & 43.5 & NE China (Wangqing) & 2 & Fan and Hooper (1991) \\
\hline 49 & 1 & 130 & 44 & NE China (Mudanjiang-Wangqing) & 10 & Liu et al (1994) \\
\hline 50 & 1 & 129.5 & 44.5 & E China (Heilongjiang) & 1 & Peng et al (1986) \\
\hline 51 & 1 & 127 & 48 & NE China (Kuandian) & 5 & Liu et al (1992) \\
\hline 51 & 1 & 127 & 48 & E China (Kundian) & 5 & Basu et al (1991) \\
\hline 52 & 1 & 126 & 50 & NE China (WEK) & 6 & Zhang et al (1995) \\
\hline 52 & 1 & 126 & 50 & $\begin{array}{l}\text { NE China (Wudalianchi- } \\
\text { Changbaishan) }\end{array}$ & 5 & Basu et al (1991) \\
\hline 52 & 1 & 126 & 50 & NE China (Wudalianchi) & 1 & Liu et al (1994) \\
\hline 112 & 2 & 29.2 & -1.4 & $\begin{array}{l}\text { East African Rift (Nyamuragira } \\
\text { Volcano) }\end{array}$ & 12 & Aoki et al (1985) \\
\hline 113 & 2 & 29.4 & -1.5 & $\begin{array}{l}\text { Karisimbi Volcano (East African } \\
\text { Rift) }\end{array}$ & 16 & De Mulder et al (1986) \\
\hline 114 & 2 & 28.9 & -2.45 & Bukavo, Zaire (East African Rift) & 4 & Auchapt et al (1987) \\
\hline 115 & 2 & 36.3 & -3.1 & $\begin{array}{l}\text { Kamituga, Zaire (East African } \\
\text { Rift) }\end{array}$ & 5 & Auchapt et al (1987) \\
\hline 116 & 2 & 36 & -2.1 & Kenya Rift (Shombole volcano) & 3 & Bell and Peterson (1991) \\
\hline 117 & 2 & 36.3 & -1.8 & $\begin{array}{l}\text { Southern Kenya Rift (East African } \\
\text { Rift) }\end{array}$ & 20 & Le Roex et al (2001) \\
\hline 173 & 3 & 246 & 30.47 & San Quintin volcanic field & 32 & Luhr et al (1995) \\
\hline 174 & 3 & 246 & 30.5 & San Quintin volcanic field & 12 & Storey et al (1989) \\
\hline 175 & 3 & 243.5 & 34.5 & $\begin{array}{l}\text { Northern Basin and Range, USA } \\
\text { (Mojave Desert) }\end{array}$ & 1 & Kempton et al (1991) \\
\hline 176 & 3 & 245 & 35 & Basin and Range, USA* & 2 & Fitton et al (1991) \\
\hline 177 & 3 & 245 & 36 & Basin and Range, USA & 12 & Feuerbach et al (1993) \\
\hline 178 & 3 & 246.9 & 35.8 & $\begin{array}{l}\text { Northeastern Transition Zone, USA } \\
\text { (Grand Canyon) }\end{array}$ & 2 & Kempton et al (1991) \\
\hline 179 & 3 & 246.6 & 34.5 & $\begin{array}{l}\text { Basin and Range, USA (Kaiser } \\
\text { Spring Field) }\end{array}$ & 4 & Moyer and Esperana (1989) \\
\hline 180 & 3 & 248.2 & 34.8 & $\begin{array}{l}\text { Southeastern Transition Zone, USA } \\
\text { (San Francisco Field) }\end{array}$ & 3 & Kempton et al (1991) \\
\hline 181 & 3 & 250 & 35 & Colorado Plateau, USA & 3 & Fitton et al (1991) \\
\hline 182 & 3 & 250.5 & 33.6 & $\begin{array}{l}\text { Southeastern Transition Zone, USA } \\
\text { (Springerville and San Carlos Field) }\end{array}$ & 5 & Kempton et al (1991) \\
\hline 183 & 3 & 243.28 & 37.07 & $\begin{array}{l}\text { Hurricane volcanic field } \\
\text { (The Divide) }\end{array}$ & 14 & Smith et al (1999) \\
\hline
\end{tabular}


Table 1. (Continued).

\begin{tabular}{|c|c|c|c|c|c|c|}
\hline $\begin{array}{c}\text { Site } \\
\#\end{array}$ & $\begin{array}{c}\text { Figure } \\
\#\end{array}$ & $\begin{array}{c}\text { Longitude } \\
\left({ }^{\circ}\right)\end{array}$ & $\begin{array}{c}\text { Latitude } \\
\left({ }^{\circ}\right)\end{array}$ & Country/Province & $\begin{array}{c}\text { No. of } \\
\text { samples }\end{array}$ & Reference \\
\hline 184 & 3 & 243.3 & 37.1 & $\begin{array}{l}\text { Hurricane volcanic field } \\
\text { (Remnants) }\end{array}$ & 5 & Smith et al (1999) \\
\hline 185 & 3 & 243.32 & 37.07 & $\begin{array}{l}\text { Hurricane volcanic field (Grass } \\
\text { Valley) }\end{array}$ & 12 & Smith et al (1999) \\
\hline 186 & 3 & 242.33 & 37.16 & $\begin{array}{l}\text { Hurricane volcanic field (Ivan's } \\
\text { Knoll) }\end{array}$ & 9 & Smith et al (1999) \\
\hline 187 & 3 & 243.3 & 37.19 & $\begin{array}{l}\text { Hurricane volcanic field (Radio } \\
\text { Towers) }\end{array}$ & 8 & Smith et al (1999) \\
\hline 188 & 3 & 243.34 & 37.17 & $\begin{array}{l}\text { Hurricane volcanic field (Volcano } \\
\text { Mountain) }\end{array}$ & 5 & Smith et al (1999) \\
\hline 189 & 3 & 243.35 & 37.18 & $\begin{array}{l}\text { Hurricane volcanic field (Cinder } \\
\text { pits) }\end{array}$ & 6 & Smith et al (1999) \\
\hline 190 & 3 & 244.1 & 37.9 & $\begin{array}{l}\text { Northern Basin and Range, USA } \\
\text { (Lunar craters) }\end{array}$ & 1 & Kempton et al (1991) \\
\hline 191 & 3 & 247 & 42.5 & Snake River Plane, USA & 2 & Fitton et al (1991) \\
\hline 191 & 3 & 247 & 42.5 & Snake River Plane, USA & 8 & Lum et al (1989) \\
\hline 192 & 3 & 247.5 & 37.5 & Transition Zone, USA & 2 & Fitton et al (1991) \\
\hline 193 & 3 & 247.7 & 37.5 & $\begin{array}{l}\text { Northeastern Transition Zone, USA } \\
\text { (Southwest Utah) }\end{array}$ & 11 & Kempton et al (1991) \\
\hline 194 & 3 & 250 & 39 & Lunar Crater Volcanic Field, USA & 15 & Lum et al (1989) \\
\hline 195 & 3 & 252.5 & 40 & $\begin{array}{l}\text { Rio Grande Rift, USA (Northwest } \\
\text { Colorado) }\end{array}$ & 3 & Gibson et al (1992) \\
\hline 196 & 3 & 253 & 39.3 & Southern Rocky Mountains, USA & 3 & Fitton et al (1991) \\
\hline 197 & 3 & 256.5 & 36.5 & Great Planes, USA & 2 & Fitton et al (1991) \\
\hline 198 & 3 & 254.4 & 36.8 & $\begin{array}{l}\text { Rio Grande Rift, USA (Latir } \\
\text { Volcanic Field) }\end{array}$ & 3 & Johnson and Lipman (1988) \\
\hline 199 & 3 & 254.4 & 35.8 & Jemez Volcanic Field, USA & 1 & Singer and Kudo (1986) \\
\hline 200 & 3 & 253.5 & 36 & $\begin{array}{l}\text { Rio Grande Rift, USA (Northern } \\
\text { New México) }\end{array}$ & 11 & Gibson et al (1992) \\
\hline 200 & 3 & 253.5 & 36 & $\begin{array}{l}\text { Rio Grande Rift (Cerros del Rio } \\
\text { Volcanic Field) }\end{array}$ & 7 & Duncker et al (1991) \\
\hline 201 & 3 & 253.49 & 35.49 & Rio Grande Rift (Santa Ana mesa) & 1 & Perry et al (1987) \\
\hline 202 & 3 & 252.3 & 35.22 & Mount Taylor Volcanic Field, USA & 5 & Perry et al (1990) \\
\hline 203 & 3 & 252.35 & 35.21 & Rio Grande Rift (Mount Taylor) & 2 & Perry et al (1987) \\
\hline 204 & 3 & 252.69 & 35.05 & Rio Grande Rift & 1 & Perry et al (1987) \\
\hline 205 & 3 & 252.6 & 34.8 & $\begin{array}{l}\text { Southeastern Transition Zone, USA } \\
\text { (Mount Taylor) }\end{array}$ & 3 & Kempton et al (1991) \\
\hline 206 & 3 & 253.2 & 34.87 & Rio Grande Rift (Cat Hills) & 1 & Perry et al (1987) \\
\hline 207 & 3 & 253.5 & 34.6 & Rio Grande Rift, USA & 3 & Kempton et al (1991) \\
\hline 208 & 3 & 253 & 33 & $\begin{array}{l}\text { Rio Grande Rift, USA (Southern } \\
\text { New México) }\end{array}$ & 13 & Gibson et al (1992) \\
\hline 209 & 3 & 252.8 & 32.5 & $\begin{array}{l}\text { Rio Grande Rift (Southern Rio } \\
\text { Grande Rift) }\end{array}$ & 15 & McMillan et al (2000) \\
\hline 210 & 3 & 252.8 & 31.5 & Rio Grande Rift, USA (Potrillo) & 3 & Kempton et al (1991) \\
\hline 211 & 3 & 250.8 & 31 & $\begin{array}{l}\text { Southern Basin and Range, USA } \\
\text { (Geronimo Field) }\end{array}$ & 1 & Kempton et al (1991) \\
\hline 300 & 4 & 242.1 & -76.7 & $\begin{array}{l}\text { Mary Byrd Land Antarctica (Crary } \\
\text { Mountains) }\end{array}$ & 6 & Panter et al (2000) \\
\hline \multicolumn{7}{|c|}{ Ocean island } \\
\hline 141 & 2 & 3.41 & -54.43 & Bouvet Islands & 6 & Le Roex and Erlank (1982) \\
\hline 141 & 2 & 3.41 & -54.43 & Bouvet Islands & 5 & Verwoerd et al (1976) \\
\hline 150 & 2 & 77.55 & -37.83 & Indian Ocean (Amsterdam Island) & 6 & Doucet et al (2004) \\
\hline 151 & 2 & 77.5 & -38.75 & Indian Ocean (St. Paul Island) & 11 & Doucet et al (2004) \\
\hline
\end{tabular}


Table 1. (Continued).

\begin{tabular}{|c|c|c|c|c|c|c|}
\hline $\begin{array}{l}\text { Site } \\
\#\end{array}$ & $\underset{\#}{\text { Figure }}$ & $\begin{array}{l}\text { Longitude } \\
\left.\qquad{ }^{\circ}\right)\end{array}$ & $\begin{array}{l}\text { Latitude } \\
\quad\left({ }^{\circ}\right)\end{array}$ & Country/Province & $\begin{array}{l}\text { No. of } \\
\text { samples }\end{array}$ & Reference \\
\hline 162 & 3 & 200.48 & 22.1 & Hawaiian Islands (Kauai) & 18 & Maaløe et al (1992) \\
\hline 163 & 3 & 202 & 21.7 & Hawaiian Islands (Koolau volcano) & 20 & Frey et al (1994) \\
\hline 164 & 3 & 203.12 & 20.86 & $\begin{array}{l}\text { Hawaiian Islands (Lanai, } \\
\text { Wawaeku) }\end{array}$ & 11 & West et al (1992) \\
\hline 165 & 3 & 203.75 & 20.72 & Hawaiian Islands (Haleakala) & 24 & Chen et al (1991) \\
\hline 165 & 3 & 203.75 & 20.72 & Hawaiian Islands (Haleakala) & 29 & Chen et al (1990) \\
\hline 166 & 3 & 203.79 & 20.39 & Hawaiian Islands (Hana) & 22 & Bergmanis et al (2000) \\
\hline 167 & 3 & 204 & 19.79 & Hawaiian Islands (Hualalai) & 2 & Norman and Garcia (1999) \\
\hline 168 & 3 & 204.46 & 19.2 & Hawaiian Islands & 15 & Lipman et al (1990) \\
\hline 169 & 3 & 204.71 & 19.42 & Hawaiian Islands (Kilauea) & 69 & Garcia et al (1992) \\
\hline 170 & 3 & 204.73 & 19.41 & Hawaiian Islands (Kilauea) & 2 & Norman and Garcıa (1999) \\
\hline 171 & 3 & 204.74 & 18.93 & Hawaiian Islands (Loihi) & 1 & Norman and Garcıa (1999) \\
\hline 172 & 3 & 249.05 & 18.78 & Socorrro Islands, Pacific Ocean & 14 & Bohrson and Reid (1995) \\
\hline 224 & 3 & 268.5 & 0.03 & $\begin{array}{l}\text { Galapagos Islands; Isabela (Volcan } \\
\text { Ecuador) }\end{array}$ & 1 & White et al (1993) \\
\hline 225 & 3 & 268.7 & 0.03 & $\begin{array}{l}\text { Galapagos Islands, Isabela (Volcan } \\
\text { Wolf) }\end{array}$ & 1 & White et al (1993) \\
\hline 226 & 3 & 268.77 & 0.25 & $\begin{array}{l}\text { Galapagos Islands, Isabela (Volcan } \\
\text { Darwin) }\end{array}$ & 1 & White et al (1993) \\
\hline 227 & 3 & 269.25 & 0.6 & Galapagos Islands (Pinta) & 5 & Cullen and McBirney (1987) \\
\hline 228 & 3 & 270.1 & 0.4 & Galapagos Islands (Genovesa) & 1 & White et al (1993) \\
\hline 263 & 3 & 343 & 65.8 & $\begin{array}{l}\text { Iceland (Sroraviti, Grjothals, } \\
\text { Gaesafjoll) }\end{array}$ & 45 & Slater et al (1998) \\
\hline 274 & 4 & 200.24 & -18.85 & French Polynesia (Aitutaki) & 7 & Dupuy et al (1989) \\
\hline 275 & 4 & 202.33 & -20 & French Polynesia (Atiu) & 10 & Dupuy et al (1989) \\
\hline 276 & 4 & 201.79 & -22 & French Polynesia (Mangaia) & 11 & Dupuy et al (1989) \\
\hline 277 & 4 & 207 & -22.6 & French Polynesia (Rimatara) & 2 & Dupuy et al (1988) \\
\hline 278 & 4 & 208.9 & -22.7 & French Polynesia (Rurutu) & 3 & Dupuy et al (1988) \\
\hline 278 & 4 & 208.9 & -22.7 & French Polynesia (Rurutu) & 2 & Dupuy et al (1989) \\
\hline 278 & 4 & 208.9 & -22.7 & French Polynesia (Rurutu) & 5 & Chauvel et al (1997) \\
\hline 279 & 4 & 210.7 & -23.3 & French Polynesia (Tubuai) & 1 & Dupuy et al (1988) \\
\hline 279 & 4 & 210.7 & -23.3 & French Polynesia (Tubuai) & 2 & Dupuy et al (1989) \\
\hline 280 & 4 & 212.2 & -24 & $\begin{array}{l}\text { French Polynesia, Pacific } \\
\text { (Raivavae) }\end{array}$ & 22 & Lassiter et al (2003) \\
\hline 280 & 4 & 212.2 & -24 & French Polynesia (Raivavae) & 3 & Dupuy et al (1988) \\
\hline 280 & 4 & 212.2 & -24 & French Polynesia (Raivavae) & 2 & Dupuy et al (1989) \\
\hline 281 & 4 & 208.34 & -19.33 & French Polynesia (Bora Bora) & 3 & Dupuy et al (1989) \\
\hline 282 & 4 & 208.5 & -16.64 & French Polynesia (Tahaa) & 2 & Dupuy et al (1989) \\
\hline 283 & 4 & 210.34 & -17.33 & French Polynesia, Pacific (Tahiti) & 9 & Hémond et al (1994) \\
\hline 284 & 4 & 210.5 & -17 & French Polynesia, Pacific (Moorea) & 1 & Hémond et al (1994) \\
\hline 285 & 4 & 211.18 & -17.57 & French Polynesia, Pacific (Teahitia) & 26 & Hémond et al (1994) \\
\hline 286 & 4 & 211.33 & -18.32 & $\begin{array}{l}\text { French Polynesia, Pacific (Moua } \\
\text { Pihaa) }\end{array}$ & 13 & Hémond et al (1994) \\
\hline 287 & 4 & 211.42 & -17.65 & French Polynesia, Pacific (Rocard) & 2 & Hémond et al (1994) \\
\hline 288 & 4 & 211.6 & -18.3 & French Polynesia (Society islands) & 13 & Cheng et al (1993) \\
\hline 289 & 4 & 211.92 & -17.88 & French Polynesia, Pacific (Mehetia) & 23 & Hémond et al (1994) \\
\hline 289 & 4 & 211.92 & -17.88 & French Polynesia (Mehetia) & 2 & Dupuy et al (1989) \\
\hline 290 & 4 & 219.3 & -8 & French Polynesia (Eiao) & 1 & Dupuy et al (1989) \\
\hline 291 & 4 & 219.34 & -7.95 & French Polynesia (Hatutu, Eiao) & 8 & Liotard et al (1986) \\
\hline 292 & 4 & 219.77 & -9.88 & French Polynesia (Ua Pou) & 2 & Dupuy et al (1989) \\
\hline
\end{tabular}


Table 1. (Continued).

\begin{tabular}{|c|c|c|c|c|c|c|}
\hline $\begin{array}{l}\text { Site } \\
\#\end{array}$ & $\begin{array}{c}\text { Figure } \\
\#\end{array}$ & $\begin{array}{l}\text { Longitude } \\
\left({ }^{\circ}\right)\end{array}$ & $\begin{array}{l}\text { Latitude } \\
\quad\left({ }^{\circ}\right)\end{array}$ & Country/Province & $\begin{array}{c}\text { No. of } \\
\text { samples }\end{array}$ & Reference \\
\hline 293 & 4 & 219.9 & -9.15 & $\begin{array}{l}\text { French Polynesia (Nuku Hiva, } \\
\text { Ua Pou) }\end{array}$ & 5 & Liotard et al (1986) \\
\hline 294 & 4 & 220.5 & -8.9 & French Polynesia (Ua Huka) & 5 & Ielsch et al (1998) \\
\hline 295 & 4 & 221 & -9.65 & $\begin{array}{l}\text { French Polynesia (Fatu Huku, } \\
\text { Hiva Ua) }\end{array}$ & 6 & Liotard et al (1986) \\
\hline 296 & 4 & 221.07 & -9.43 & French Polynesia (Fatu Huku) & 1 & Dupuy et al (1989) \\
\hline 297 & 4 & 215.59 & -27.5 & French Polynesia, Pacific (Rapa) & 4 & Hémond et al (1994) \\
\hline 297 & 4 & 215.59 & -27.5 & French Polynesia, Pacific (Rapa) & 10 & Lassiter et al (2003) \\
\hline 297 & 4 & 215.59 & -27.5 & French Polynesia (Rapa) & 4 & Dupuy et al (1988) \\
\hline 297 & 4 & 215.59 & -27.5 & French Polynesia (Rapa) & 1 & Dupuy et al (1989) \\
\hline 298 & 4 & 219.67 & -28.12 & $\begin{array}{l}\text { French Polynesia, Pacific } \\
\text { (Ra Seamount) }\end{array}$ & 1 & Hémond et al (1994) \\
\hline 299 & 4 & 219.5 & -29 & $\begin{array}{l}\text { French Polynesia, Pacific } \\
\text { (Macdonald) }\end{array}$ & 29 & Hémond et al (1994) \\
\hline 299 & 4 & 219.5 & -29 & French Polynesia, Pacific (Marotiri) & 2 & Hémond et al (1994) \\
\hline 299 & 4 & 219.5 & -29 & French Polynesia (Macdonald) & 3 & Dupuy et al (1988) \\
\hline 299 & 4 & 219.5 & -29 & French Polynesia (Macdonald) & 1 & Dupuy et al (1989) \\
\hline 326 & 4 & 268.9 & -0.55 & $\begin{array}{l}\text { Galapagos Islands, Isabela (Sierra } \\
\text { Negra) }\end{array}$ & 1 & White et al (1993) \\
\hline 327 & 4 & 268.94 & -0.42 & $\begin{array}{l}\text { Galapagos Islands, Isabela (Volcan } \\
\text { Alcedo) }\end{array}$ & 1 & White et al (1993) \\
\hline 328 & 4 & 269.33 & -0.6 & Galapagos Islands (Pinzon) & 1 & White et al (1993) \\
\hline 329 & 4 & 269.33 & -0.25 & Galapagos Islands (Santiago) & 3 & White et al (1993) \\
\hline 330 & 4 & 269.56 & -1.29 & Galapagos Islands (Floreana) & 1 & White et al (1993) \\
\hline 331 & 4 & 269.77 & -0.6 & Galapagos Islands (Santa Cruz) & 4 & White et al (1993) \\
\hline 332 & 4 & 270.35 & -1.4 & Galapagos Islands (Española) & 1 & White et al (1993) \\
\hline 333 & 4 & 270.55 & -0.87 & Galapagos Islands (San Cristóbal) & 3 & Geist et al (1986) \\
\hline 334 & 4 & 270.7 & -0.75 & Galapagos Islands (San Cristóbal) & 1 & White et al (1993) \\
\hline \multicolumn{7}{|c|}{ MORB } \\
\hline 1 & 1 & 0.51 & 72.2 & Mid Atlantic Ridge, Mohns ridge & 6 & Schilling et al (1983) \\
\hline 142 & 2 & 22.92 & -52.79 & $\begin{array}{l}\text { Indian Ridge (central southwest; } \\
17-26^{\circ} \mathrm{E} \text { ) }\end{array}$ & 4 & Mahoney et al (1992) \\
\hline 143 & 2 & 33.54 & -46.56 & $\begin{array}{l}\text { Indian Ridge (central southwest; } \\
\text { Andrew Bain-Prince Edward } \\
\text { fracture zone area) }\end{array}$ & 3 & Mahoney et al (1992) \\
\hline 144 & 2 & 35.85 & -44.82 & $\begin{array}{l}\text { Indian Ridge (central southwest; } \\
36-39^{\circ} \mathrm{E} \text { ) }\end{array}$ & 2 & Mahoney et al (1992) \\
\hline 145 & 2 & 41.99 & -42.69 & $\begin{array}{l}\text { Indian Ridge (central southwest; } \\
\text { Discovery II fracture zone) }\end{array}$ & 1 & Mahoney et al (1992) \\
\hline 146 & 2 & 46.68 & -38.54 & $\begin{array}{l}\text { Indian Ridge (central southwest; } \\
\text { Indomed fracture zone to } 50^{\circ} \mathrm{E} \text { ) }\end{array}$ & 2 & Mahoney et al (1992) \\
\hline 147 & 2 & 66.75 & -27.08 & $\begin{array}{l}\text { Indian Ridge (Southwest ridge, } \\
\text { Indian Ocean triple junction; Leg 5) }\end{array}$ & 3 & Price et al (1986) \\
\hline 148 & 2 & 70.04 & -25.5 & $\begin{array}{l}\text { Indian Ridge (Indian Ocean triple } \\
\text { junction; Leg 6) }\end{array}$ & 8 & Price et al (1986) \\
\hline 149 & 2 & 70.07 & -25.38 & Indian Ridge (central Indian ridge) & 12 & Dosso et al (1988) \\
\hline 212 & 3 & 251.27 & 22.87 & East Pacific Rise (Site 483) & 2 & Cambon et al (1979) \\
\hline 213 & 3 & 252 & 22.77 & East Pacific Rise (Site 482) & 3 & Cambon et al (1979) \\
\hline 214 & 3 & 252.1 & 22.72 & East Pacific Rise (Site 485) & 1 & Cambon et al (1979) \\
\hline 215 & 3 & 252.25 & 9.82 & East Pacific Rise $9^{\circ}-10^{\circ} \mathrm{N}$ & 6 & Sims et al (2003) \\
\hline 216 & 3 & 255.69 & 9.83 & East Pacific Rise $9^{\circ}-10^{\circ} \mathrm{N}$ & 4 & Sims et al (2003) \\
\hline 217 & 3 & 255.7 & 9.84 & East Pacific Rise $9^{\circ}-10^{\circ} \mathrm{N}$ & 2 & Sims et al (2003) \\
\hline
\end{tabular}


Table 1. (Continued).

\begin{tabular}{|c|c|c|c|c|c|c|}
\hline $\begin{array}{l}\text { Site } \\
\#\end{array}$ & $\begin{array}{c}\text { Figure } \\
\quad \#\end{array}$ & $\begin{array}{l}\text { Longitude } \\
\left({ }^{\circ}\right)\end{array}$ & $\begin{array}{l}\text { Latitude } \\
\left({ }^{\circ}\right)\end{array}$ & Country/Province & $\begin{array}{c}\text { No. of } \\
\text { samples }\end{array}$ & Reference \\
\hline 218 & 3 & 255.73 & 9.84 & East Pacific Rise $9^{\circ}-10^{\circ} \mathrm{N}$ & 3 & Sims et al (2003) \\
\hline 219 & 3 & 255.71 & 2.67 & $\begin{array}{l}\text { East Pacific Rise (RRR triple } \\
\text { junction) }\end{array}$ & 10 & Lonsdale et al (1992) \\
\hline 220 & 3 & 261.93 & 2.28 & $\begin{array}{l}\text { Galapagos Spreading Center } \\
\left(96^{\circ}-101^{\circ} \mathrm{W}\right)\end{array}$ & 6 & Schilling et al (1982) \\
\hline 221 & 3 & 262.14 & 2.24 & $\begin{array}{l}\text { Galapagos Spreading Center } \\
\left(96^{\circ}-101^{\circ} \mathrm{W}\right)\end{array}$ & 1 & Verma and Schilling (1982) \\
\hline 222 & 3 & 266.85 & 2.29 & $\begin{array}{l}\text { Galapagos Spreading Center } \\
\left(91^{\circ}-95^{\circ} \mathrm{W}\right)\end{array}$ & 16 & Verma and Schilling (1982) \\
\hline 223 & 3 & 267.49 & 2.14 & $\begin{array}{l}\text { Galapagos Spreading Center } \\
\left(91^{\circ}-95^{\circ} \mathrm{W}\right)\end{array}$ & 2 & Schilling et al (1982) \\
\hline 229 & 3 & 270.18 & 0.39 & $\begin{array}{l}\text { Genovesa Ridge (Base of a large } \\
\text { cone; dredge 22) }\end{array}$ & 9 & Harpp et al (2003) \\
\hline 230 & 3 & 270.21 & 0.46 & $\begin{array}{l}\text { Genovesa Ridge (Base of the N. } \\
\text { ridge flank; dredge } 23 \text { ) }\end{array}$ & 7 & Harpp et al (2003) \\
\hline 231 & 3 & 271.65 & 0.89 & $\begin{array}{l}\text { Galapagos Spreading Center } \\
\left(83^{\circ}-90^{\circ} \mathrm{W}\right)\end{array}$ & 19 & Verma and Schilling (1982) \\
\hline 232 & 3 & 273.77 & 1.77 & $\begin{array}{l}\text { Galapagos Spreading Center } \\
\left(83^{\circ}-90^{\circ} \mathrm{W}\right)\end{array}$ & 13 & Schilling et al (1982) \\
\hline 243 & 3 & 319.17 & 10.62 & Mid Atlantic Ridge $\left(10-13^{\circ} \mathrm{N}\right)$ & 2 & Bougault et al (1988) \\
\hline 244 & 3 & 315.44 & 13.61 & Mid Atlantic Ridge $\left(10-15.5^{\circ} \mathrm{N}\right)$ & 25 & Dosso et al (1993) \\
\hline 245 & 3 & 315 & 14.06 & Mid Atlantic Ridge $\left(13.1-14.4^{\circ} \mathrm{N}\right)$ & 6 & Bougault et al (1988) \\
\hline 246 & 3 & 315.16 & 14.5 & Mid Atlantic Ridge $\left(14.5^{\circ} \mathrm{N}\right)$ & 1 & Bougault et al (1988) \\
\hline 247 & 3 & 313.45 & 17.41 & Mid Atlantic Ridge $\left(15.6-18.9^{\circ} \mathrm{N}\right)$ & 27 & Dosso et al (1993) \\
\hline 248 & 3 & 314.71 & 20.57 & Mid Atlantic Ridge $\left(19-22.9^{\circ} \mathrm{N}\right)$ & 39 & Dosso et al (1993) \\
\hline 249 & 3 & 315.1 & 23.1 & $\begin{array}{l}\text { MAR, Kane fracture zone (Older } \\
\text { walls, southern rift valley) }\end{array}$ & 4 & Bryan et al (1981) \\
\hline 250 & 3 & 314.97 & 23.61 & Mid Atlantic Ridge $\left(23-25^{\circ} \mathrm{N}\right)$ & 25 & Dosso et al (1993) \\
\hline 251 & 3 & 314.97 & 23.62 & $\begin{array}{l}\text { MAR, Kane fracture zone (South } \\
\text { wall) }\end{array}$ & 7 & Bryan et al (1981) \\
\hline 252 & 3 & 315.01 & 23.65 & $\begin{array}{l}\text { MAR, Kane fracture zone (North } \\
\text { older walls) }\end{array}$ & 1 & Bryan et al (1981) \\
\hline 253 & 3 & 315.14 & 23.57 & $\begin{array}{l}\text { MAR, Kane fracture zone } \\
\text { (Southern rift valley) }\end{array}$ & 4 & Bryan et al (1981) \\
\hline 254 & 3 & 313.61 & 23.85 & $\begin{array}{l}\text { MAR, Kane fracture zone (South } \\
\text { older walls) }\end{array}$ & 1 & Bryan et al (1981) \\
\hline 255 & 3 & 313.79 & 23.91 & $\begin{array}{l}\text { MAR, Kane fracture zone (North } \\
\text { wall) }\end{array}$ & 4 & Bryan et al (1981) \\
\hline 256 & 3 & 313.71 & 23.95 & $\begin{array}{l}\text { MAR, Kane fracture zone } \\
\text { (Northern rift valley) }\end{array}$ & 6 & Bryan et al (1981) \\
\hline 257 & 3 & 316.7 & 28.91 & $\begin{array}{l}\text { MAR, Kane fracture zone (Older } \\
\text { walls, northern rift valley) }\end{array}$ & 2 & Bryan et al (1981) \\
\hline 258 & 3 & 326.34 & 36.42 & $\begin{array}{l}\text { Mid Atlantic Ridge (AMAR rift } \\
\text { valley) }\end{array}$ & 3 & Le Roex et al (1996) \\
\hline 259 & 3 & 326.7 & 36.73 & $\begin{array}{l}\text { Mid Atlantic Ridge (FAMOUS rift } \\
\text { valley, Narrowgate) }\end{array}$ & 2 & Le Roex et al (1996) \\
\hline 260 & 3 & 327.51 & 38.73 & Mid Atlantic Ridge $\left(52-28^{\circ} \mathrm{N}\right)$ & 71 & Schilling et al (1983) \\
\hline 261 & 3 & 327.6 & 52.74 & $\begin{array}{l}\text { Mid Atlantic Ridge, Gibbs fracture } \\
\text { zone }\end{array}$ & 4 & Schilling et al (1983) \\
\hline 262 & 3 & 333.51 & 61.81 & $\begin{array}{l}\text { Mid Atlantic Ridge, Reykjanes } \\
\text { ridge }\end{array}$ & 40 & Schilling et al (1983) \\
\hline 263 & 3 & 342.92 & 68.42 & $\begin{array}{l}\text { Mid Atlantic Ridge, Kolbeinsey } \\
\text { Ridge }\end{array}$ & 26 & Schilling et al (1983) \\
\hline
\end{tabular}


Table 1. (Continued).

\begin{tabular}{|c|c|c|c|c|c|c|}
\hline $\begin{array}{l}\text { Site } \\
\#\end{array}$ & $\underset{\#}{\text { Figure }}$ & $\begin{array}{l}\text { Longitude } \\
\qquad\left(^{\circ}\right)\end{array}$ & $\begin{array}{l}\text { Latitude } \\
\left({ }^{\circ}\right)\end{array}$ & Country/Province & $\begin{array}{c}\text { No. of } \\
\text { samples }\end{array}$ & Reference \\
\hline 264 & 3 & 354.1 & 71.15 & $\begin{array}{l}\text { Mid Atlantic Ridge (Mohns Ridge) } \\
\text { Platform ridge }\end{array}$ & 2 & Haase et al (1996) \\
\hline 266 & 3 & 358.31 & 72.38 & $\begin{array}{l}\text { Mid Atlantic Ridge (Mohns Ridge) } \\
1.69^{\circ} \mathrm{W}\end{array}$ & 1 & Haase et al (1996) \\
\hline 267 & 3 & 359.28 & 72.01 & $\begin{array}{l}\text { Mid Atlantic Ridge (Mohns Ridge) } \\
0.54^{\circ} \mathrm{W}\end{array}$ & 5 & Haase et al (1996) \\
\hline 301 & 4 & 239.92 & -36.85 & $\begin{array}{l}\text { Chile Ridge, North Chile Ridge } \\
\text { (Segment 3) }\end{array}$ & 15 & Bach et al (1996) \\
\hline 302 & 4 & 249.09 & -37.77 & Pacific Antarctic Ridge (PAR axis) & 1 & Hekinian et al (1997) \\
\hline 303 & 4 & 249.2 & -37.49 & Pacific Antarctic Ridge (PAR axis) & 2 & Hekinian et al (1997) \\
\hline 304 & 4 & 249.37 & -36.91 & Pacific Antarctic Ridge (PAR axis) & 1 & Hekinian et al (1997) \\
\hline 305 & 4 & 240.75 & -30.67 & East Pacific Rise & 1 & Engel and Engel (1964) \\
\hline 306 & 4 & 242.5 & -28.98 & East Pacific Rise & 1 & Engel and Engel (1964) \\
\hline 307 & 4 & 248.06 & -28.77 & East Pacific Rise $6^{\circ}-30^{\circ} \mathrm{S}$ & 3 & Bach et al (1994) \\
\hline 308 & 4 & 248.09 & -23.39 & $\begin{array}{l}\text { East Pacific Rise (Easter } \\
\text { microplate; East rift) }\end{array}$ & 2 & Hekinian et al (1996) \\
\hline 309 & 4 & 248.48 & -23.31 & $\begin{array}{l}\text { East Pacific Rise (Easter } \\
\text { microplate; Pito seamount) }\end{array}$ & 3 & Hekinian et al (1996) \\
\hline 310 & 4 & 246.32 & -23.24 & $\begin{array}{l}\text { East Pacific Rise (Easter } \\
\text { microplate; microplate interior) }\end{array}$ & 1 & Hekinian et al (1996) \\
\hline 311 & 4 & 248.34 & -23 & $\begin{array}{l}\text { East Pacific Rise (Easter } \\
\text { microplate; Pito deep) }\end{array}$ & 1 & Hekinian et al (1996) \\
\hline 312 & 4 & 247.34 & -21.99 & East Pacific Rise $6^{\circ}-30^{\circ} \mathrm{S}$ & 7 & Bach et al (1994) \\
\hline 313 & 4 & 246.32 & -21.96 & $\begin{array}{l}\text { East Pacific Rise (Easter } \\
\text { microplate; thrust-fault) }\end{array}$ & 1 & Hekinian et al (1996) \\
\hline 314 & 4 & 246.67 & -18.42 & East Pacific Rise & 1 & Engel and Engel (1964) \\
\hline 315 & 4 & 245.63 & -18.25 & East Pacific Rise $6^{\circ}-30^{\circ} \mathrm{S}$ & 10 & Bach et al (1994) \\
\hline 316 & 4 & 246.62 & -14.62 & East Pacific Rise $6^{\circ}-30^{\circ} \mathrm{S}$ & 7 & Bach et al (1994) \\
\hline 317 & 4 & 249.05 & -12.87 & East Pacific Rise & 1 & Engel and Engel (1964) \\
\hline 318 & 4 & 247.36 & -11.11 & East Pacific Rise $6^{\circ}-30^{\circ} \mathrm{S}$ & 6 & Bach et al (1994) \\
\hline 319 & 4 & 251.84 & -7.78 & East Pacific Rise & 1 & Engel and Engel (1964) \\
\hline 320 & 4 & 252.03 & -8.33 & $\begin{array}{l}\text { East Pacific Rise }\left(8^{\circ} 17^{\prime} \mathrm{S}, \text { dredge }\right. \\
139)\end{array}$ & 1 & Hall and Sinton (1996) \\
\hline 321 & 4 & 252.04 & -8.25 & $\begin{array}{l}\text { East Pacific Rise }\left(8^{\circ} 17^{\prime} \mathrm{S}, \text { dredge }\right. \\
145)\end{array}$ & 1 & Hall and Sinton (1996) \\
\hline 322 & 4 & 252.06 & -8.15 & $\begin{array}{l}\text { East Pacific Rise }\left(8^{\circ} 17^{\prime} \mathrm{S} \text {, dredge }\right. \\
142)\end{array}$ & 1 & Hall and Sinton (1996) \\
\hline 323 & 4 & 252.1 & -8.29 & $\begin{array}{l}\text { East Pacific Rise }\left(8^{\circ} 17^{\prime} \mathrm{S}, \text { dredge }\right. \\
140)\end{array}$ & 1 & Hall and Sinton (1996) \\
\hline 324 & 4 & 252.16 & -8.18 & $\begin{array}{l}\text { East Pacific Rise }\left(8^{\circ} 17^{\prime} \mathrm{S}, \text { dredge }\right. \\
146)\end{array}$ & 1 & Hall and Sinton (1996) \\
\hline 325 & 4 & 249.53 & -7.31 & East Pacific Rise $6^{\circ}-30^{\circ} \mathrm{S}$ & 5 & Bach et al (1994) \\
\hline 335 & 4 & 267.57 & -21.82 & $\begin{array}{l}\text { East Pacific Rise (Easter } \\
\text { microplate; N. of thrust-fault) }\end{array}$ & 1 & Hekinian et al (1996) \\
\hline 336 & 4 & 263.29 & -38.04 & $\begin{array}{l}\text { Chile Ridge, North Chile Ridge } \\
\text { (Segment 2) }\end{array}$ & 11 & Bach et al (1996) \\
\hline 337 & 4 & 266.26 & -38.71 & $\begin{array}{l}\text { Chile Ridge, North Chile Ridge } \\
\text { (Segment 1) }\end{array}$ & 12 & Bach et al (1996) \\
\hline 338 & 4 & 267.57 & -55.67 & $\begin{array}{l}\text { America-Antarctica Ridge (Conrad } \\
\text { fracture zone, near Bouvet triple } \\
\text { junction) }\end{array}$ & 9 & Le Roex and Dick (1981) \\
\hline 342 & 4 & 357.9 & -54.17 & $\begin{array}{l}\text { Mid Atlantic Ridge (Ridge axis and } \\
\text { fracture zones; } 54^{\circ} \mathrm{S} \text { ) }\end{array}$ & 20 & Le Roex et al (1987) \\
\hline
\end{tabular}


Table 1. (Continued).

\begin{tabular}{|c|c|c|c|c|c|c|}
\hline $\begin{array}{l}\text { Site } \\
\#\end{array}$ & $\underset{\text { Figure }}{\#}$ & $\begin{array}{l}\text { Longitude } \\
\left(^{\circ}\right)\end{array}$ & $\begin{array}{l}\text { Latitude } \\
\quad\left({ }^{\circ}\right)\end{array}$ & Country/Province & $\begin{array}{c}\text { No. of } \\
\text { samples }\end{array}$ & Reference \\
\hline 343 & 4 & 355.48 & -52.63 & $\begin{array}{l}\text { Mid Atlantic Ridge (Fracture zones; } \\
52^{\circ} \mathrm{S} \text { ) }\end{array}$ & 11 & Le Roex et al (1987) \\
\hline 344 & 4 & 354.28 & -51.45 & $\begin{array}{l}\text { Mid Atlantic Ridge (Fracture zones; } \\
51^{\circ} \mathrm{S} \text { ) }\end{array}$ & 9 & Le Roex et al (1987) \\
\hline 345 & 4 & 353.96 & -51.16 & $\begin{array}{l}\text { MAR, southern region } \\
\left(50.3-52.46^{\circ} \mathrm{S}\right)\end{array}$ & 16 & Le Roux et al (2002b) \\
\hline 346 & 4 & 353.95 & -51.11 & $\begin{array}{l}\text { MAR, southern region } \\
\left(50.3-54.4^{\circ} \mathrm{S}\right)\end{array}$ & 66 & Le Roux et al (2002a) \\
\hline 347 & 4 & 352.02 & -49.69 & $\begin{array}{l}\text { MAR, southern region } \\
\left(49.3-50.0^{\circ} \mathrm{S}\right)\end{array}$ & 39 & Le Roux et al (2002a) \\
\hline 348 & 4 & 351.97 & -49.57 & $\begin{array}{l}\text { MAR, southern region } \\
\left(49.3-50.0^{\circ} \mathrm{S}\right)\end{array}$ & 6 & Le Roux et al (2002b) \\
\hline 349 & 4 & 349.92 & -48.34 & $\begin{array}{l}\text { MAR, southern region } \\
\left(47.4-49.2^{\circ} \mathrm{S}\right)\end{array}$ & 14 & Le Roux et al (2002b) \\
\hline 350 & 4 & 349.91 & -48.2 & $\begin{array}{l}\text { MAR, southern region } \\
\left(47.4-49.2^{\circ} \mathrm{S}\right)\end{array}$ & 71 & Le Roux et al (2002a) \\
\hline 351 & 4 & 347.1 & -47.12 & $\begin{array}{l}\text { MAR, central region } \\
\left(46.9-47.45^{\circ} \mathrm{S}\right)\end{array}$ & 6 & Le Roux et al (2002b) \\
\hline 352 & 4 & 346.57 & -47.06 & $\begin{array}{l}\text { MAR, central region } \\
\left(46.9-47.45^{\circ} \mathrm{S}\right)\end{array}$ & 14 & Le Roux et al (2002a) \\
\hline 353 & 4 & 346.15 & -46.14 & $\begin{array}{l}\text { MAR, northern region } \\
\left(43.4-46.6^{\circ} \mathrm{S}\right)\end{array}$ & 11 & Le Roux et al (2002b) \\
\hline 354 & 4 & 345.95 & -45.97 & $\begin{array}{l}\text { MAR, northern region } \\
\left(43.4-46.6^{\circ} \mathrm{S}\right)\end{array}$ & 33 & Le Roux et al (2002a) \\
\hline 355 & 4 & 343.72 & -42.8 & $\begin{array}{l}\text { MAR, northern region } \\
\left(40.6-44.8^{\circ} \mathrm{S}\right)\end{array}$ & 41 & Le Roux et al (2002a) \\
\hline 356 & 4 & 343.78 & -42.75 & $\begin{array}{l}\text { MAR, northern region } \\
\left(40.6-44.8^{\circ} \mathrm{S}\right)\end{array}$ & 8 & Le Roux et al (2002b) \\
\hline
\end{tabular}

Table 2. Sample sizes in the training and testing sets and comparison with our earlier work.

\begin{tabular}{|c|c|c|c|c|c|c|c|}
\hline \multirow{2}{*}{\multicolumn{2}{|c|}{ Group }} & \multicolumn{2}{|c|}{ Training set } & \multicolumn{2}{|c|}{ Testing set } & \multicolumn{2}{|c|}{ Total } \\
\hline & & & Agrawal et al & & Agrawal et & & Agrawal et al \\
\hline Name & Number & This work & (2004) & This work & $(2004)$ & This work & (2004) \\
\hline IAB & (1) & 363 & 224 & 100 & 25 & 463 & 249 \\
\hline CRB & $(2)$ & 671 & 209 & 100 & 25 & 771 & 234 \\
\hline OIB & (3) & 472 & 227 & 100 & 25 & 572 & 252 \\
\hline MORB & (4) & 826 & 399 & 100 & 25 & 926 & 424 \\
\hline Total & & 2332 & 1059 & 400 & 100 & 2732 & 1159 \\
\hline
\end{tabular}

$\mathrm{IAB}=$ island arc basic rock; $\mathrm{CRB}=$ continental rift basic rock; OIB = ocean island basic rock and $\mathrm{MORB}=$ midocean ridge basic rock. The numbers in parentheses are group numbers discussed in the text.

turn, depends on the resulting rock type. Because most persons have not used SINCLAS, we once again emphasize that it is not easy to rely on a hand calculator or some simple computer program because the proper Fe-adjustment requires an iterative process. To remind the user about this basic need, we have called the new major-element array with the subscript adj, such as $\left(\mathrm{SiO}_{2}\right)_{\text {adj }}$ for $\mathrm{SiO}_{2}$, and so on.

\section{Data processing}

\subsection{The need for transformation of the compositional data}

As pointed out in the Introduction section, we should think in terms of ratios and abandon the traditional way of looking at the compositions in terms of percentages (major-elements), or ppm, 
Table 3. Means and standard deviations of log-transformed major element ratios for the four group sets IAB-CRB-OIB-MORB.

\begin{tabular}{|c|c|c|c|c|c|c|c|c|}
\hline \multirow{2}{*}{$\begin{array}{l}\log _{e} \text {-transformed } \\
\text { ratio variable }\end{array}$} & \multicolumn{2}{|c|}{$\begin{array}{c}\text { IAB (1) } \\
(n=463)\end{array}$} & \multicolumn{2}{|c|}{$\begin{array}{l}\text { CRB (2) } \\
(n=771)\end{array}$} & \multicolumn{2}{|c|}{$\begin{array}{c}\text { OIB (3) } \\
(n=572)\end{array}$} & \multicolumn{2}{|c|}{$\begin{array}{l}\operatorname{MORB}(4) \\
(n=926)\end{array}$} \\
\hline & mean & $\mathrm{s}$ & mean & $\mathrm{s}$ & mean & $\mathrm{s}$ & mean & $\mathrm{s}$ \\
\hline $\ln \left(\mathrm{TiO}_{2} / \mathrm{SiO}_{2}\right)_{\mathrm{adj}}$ & -4.03 & 0.27 & -3.05 & 0.30 & -2.90 & 0.43 & -3.56 & 0.26 \\
\hline $\ln \left(\mathrm{Al}_{2} \mathrm{O}_{3} / \mathrm{SiO}_{2}\right)_{\text {adj }}$ & -1.06 & 0.12 & -1.17 & 0.10 & -1.24 & 0.16 & -1.19 & 0.08 \\
\hline $\ln \left(\mathrm{Fe}_{2} \mathrm{O}_{3} / \mathrm{SiO}_{2}\right)_{\text {adj }}$ & -3.38 & 0.15 & -3.08 & 0.20 & -3.15 & 0.21 & -3.40 & 0.14 \\
\hline $\ln \left(\mathrm{FeO} / \mathrm{SiO}_{2}\right)_{\mathrm{adj}}$ & -1.82 & 0.14 & -1.64 & 0.19 & -1.58 & 0.17 & -1.80 & 0.14 \\
\hline $\ln \left(\mathrm{MnO} / \mathrm{SiO}_{2}\right)_{\mathrm{adj}}$ & -5.61 & 0.20 & -5.58 & 0.20 & -5.56 & 0.16 & -5.69 & 0.18 \\
\hline $\ln \left(\mathrm{MgO} / \mathrm{SiO}_{2}\right)_{\mathrm{adj}}$ & -2.02 & 0.39 & -1.81 & 0.36 & -1.8 & 0.5 & -1.88 & 0.15 \\
\hline $\ln \left(\mathrm{CaO} / \mathrm{SiO}_{2}\right)_{\mathrm{adj}}$ & -1.50 & 0.14 & -1.61 & 0.20 & -1.51 & 0.18 & -1.48 & 0.09 \\
\hline $\ln \left(\mathrm{Na}_{2} \mathrm{O} / \mathrm{SiO}_{2}\right)_{\mathrm{adj}}$ & -3.02 & 0.24 & -2.67 & 0.25 & -2.91 & 0.33 & -2.95 & 0.15 \\
\hline $\ln \left(\mathrm{K}_{2} \mathrm{O} / \mathrm{SiO}_{2}\right)_{\mathrm{adj}}$ & -4.4 & 0.8 & -3.6 & 0.6 & -4.4 & 1.0 & -5.7 & 0.9 \\
\hline $\ln \left(\mathrm{P}_{2} \mathrm{O}_{5} / \mathrm{SiO}_{2}\right)_{\mathrm{adj}}$ & -5.7 & 0.7 & -4.5 & 0.5 & -4.9 & 0.7 & -5.83 & 0.43 \\
\hline
\end{tabular}

Subscript adj refers to the concentrations recalculated to an anhydrous $100 \%$ adjusted basis using computer program SINCLAS employing the Middlemost (1989) recommendation for iron-oxidation ratio adjustment (Verma et al 2002). This was done prior to the log transformation of the data. Rounding of mean values was done according to the corresponding standard deviation (s) data (Verma 2005). Tectonic settings, group numbers, and the number of compiled samples $(n)$ are the same as in table 2.

or other concentration units (trace elements). The percentage data occupy a restricted region of sample space; the ratios allow them to spread more freely through this space. In fact, the calculation of log-ratios has the consequence of freeing space values from a restricted range to vary between $-\infty$ and $+\infty$, which is a highly desirable property for most statistical inferences including the discriminant analysis. Aitchison (1986) proposed that compositional data should be expressed as the covariances of log-ratios of the variables rather than the raw percentages. Thus, we decided to ratio every variable against a common divisor. According to Aitchison (1986) the choice of a variable as the divisor is immaterial because only the structure of the ratio matrix is important. For our major-element data, we decided to use the most abundant part $\mathrm{SiO}_{2}$ as the divisor and then to transform the ratio data using $\log _{e}$ (natural logarithm base e, or $\ln$ ). In fact, we experimented with several other variables as the divisor and obtained similar results. We did not experiment with other types of logarithms such as $\log _{10}$, but they should yield similar final results. Thus, our final variables for the discriminant analysis were (one less than the original variables): $\ln \left(\mathrm{TiO}_{2} / \mathrm{SiO}_{2}\right)_{\text {adj }}, \quad \ln \left(\mathrm{Al}_{2} \mathrm{O}_{3} / \mathrm{SiO}_{2}\right)_{\text {adj }}, \quad \ln \left(\mathrm{Fe}_{2} \mathrm{O}_{3} /\right.$ $\left.\mathrm{SiO}_{2}\right)_{\text {adj }}, \quad \ln \left(\mathrm{FeO} / \mathrm{SiO}_{2}\right)_{\text {adj }}, \quad \ln \left(\mathrm{MnO} / \mathrm{SiO}_{2}\right)_{\text {adj }}$, $\ln \left(\mathrm{MgO} / \mathrm{SiO}_{2}\right)_{\text {adj }}, \quad \ln \left(\mathrm{CaO} / \mathrm{SiO}_{2}\right)_{\text {adj }} \quad \ln \left(\mathrm{Na}_{2} \mathrm{O} /\right.$ $\left.\mathrm{SiO}_{2}\right)_{\text {adj }}, \ln \left(\mathrm{K}_{2} \mathrm{O} / \mathrm{SiO}_{2}\right)_{\text {adj }}$, and $\ln \left(\mathrm{P}_{2} \mathrm{O}_{5} / \mathrm{SiO}_{2}\right)_{\text {adj }}$. Although the adjustment factor $($ adj $)$ will cancel out from the ratios of concentration data, this is to remind the reader that the calculation of $\mathrm{Fe}_{2} \mathrm{O}_{3}$ and $\mathrm{FeO}$ using the Middlemost option (Verma et al 2002) is still a recommended, probably a mandatory step during the application procedure.

\subsection{Comparison of log-transformed major-element ratios of basic rocks from different tectonic settings}

Instead of reporting the average major-element compositions and corresponding standard deviation values, we present the basic statistical information of $\log _{\mathrm{e}}$-transformed major-element ratios of the four tectonic types of basic rocks (table 3 ). Note all mean values are negative because the divisor $\left(\mathrm{SiO}_{2}\right)$ used was the most abundant element rendering all ratios to be $<1$, and consequently their logarithms to be negative. The Student t test (for ANOVA or F value test, see below) revealed that for most ratios, there were statistically significant differences between the mean values from different tectonic settings. However, the smallest differences were observed between the CRB and OIB, or the OIB and MORB groups, making any simple bivariate or ternary plots of these $\log _{\mathrm{e}}{ }^{-}$ transformed ratios unsuccessful in discriminating between these four tectonic settings (plots not shown). Therefore, multivariate techniques such as linear discriminant analysis, were actually required to obtain meaningful discrimination of these settings.

Another way of looking at these data is to determine if the geochemical variation within the group is low compared to that between groups. Table 4 shows Wilk's lambda, also called U-statistic, and represents the ratio of the within-group sum of 
Table 4. Test for equality of group means.

\begin{tabular}{lccc}
\hline $\begin{array}{l}\mathrm{Log}_{\mathrm{e}} \text {-transformed } \\
\text { ratio variable }\end{array}$ & Wilks' Lambda & F-ratio & Significance \\
\hline $\ln \left(\mathrm{TiO}_{2} / \mathrm{SiO}_{2}\right)_{\text {adj }}$ & 0.3991 & 1168 & 0.0000 \\
$\ln \left(\mathrm{Al}_{2} \mathrm{O}_{3} / \mathrm{SiO}_{2}\right)_{\text {adj }}$ & 0.8100 & 182 & 0.0000 \\
$\ln \left(\mathrm{Fe}_{2} \mathrm{O}_{3} / \mathrm{SiO}_{2}\right)_{\text {adj }}$ & 0.6005 & 516 & 0.0000 \\
$\ln \left(\mathrm{FeO} / \mathrm{SiO}_{2}\right)_{\text {adj }}$ & 0.7320 & 284 & 0.0000 \\
$\ln \left(\mathrm{MnO} / \mathrm{SiO}_{2}\right)_{\text {adj }}$ & 0.9146 & 73 & 0.0000 \\
$\ln \left(\mathrm{MgO} / \mathrm{SiO}_{2}\right)_{\text {adj }}$ & 0.9392 & 50 & 0.0000 \\
$\ln \left(\mathrm{CaO} / \mathrm{SiO}_{2}\right)_{\text {adj }}$ & 0.8910 & 95 & 0.0000 \\
$\ln \left(\mathrm{Na}_{2} \mathrm{O} / \mathrm{SiO}_{2}\right)_{\text {adj }}$ & 0.7627 & 241 & 0.0000 \\
$\ln \left(\mathrm{K}_{2} \mathrm{O} / \mathrm{SiO}_{2}\right)_{\text {adj }}$ & 0.4777 & 848 & 0.0000 \\
$\ln \left(\mathrm{P}_{2} \mathrm{O}_{5} / \mathrm{SiO}_{2}\right)_{\text {adj }}$ & 0.4979 & 783 & 0.0000 \\
\hline $\mathrm{Becalse}^{\mathrm{SiO}}{ }_{2}$ ) & & &
\end{tabular}

Because $\mathrm{SiO}_{2}$ was used for log transformations, this row is absent. Wilks' Lambda (U-statistic) and univariate F-ratio with degrees of freedom, $\mathrm{DF} 1=g-1=4-1=3$ and DF2 $=n-g=2332-4=2328$, where $g$ is the number of groups and $n$ is the total number of samples.

squares to the total sum of squares (therefore, this statistic can have values between 0 and 1 ). A lambda of 1 is observed when all group means are equal, whereas small values close to zero signify that within-group variability is small compared to the total variability. In other words, large values of lambda indicate that group means are not statistically different, whereas small values indicate the opposite. The Wilk's lambda values for most $\log _{\mathrm{e}}-$ transformed major-element ratios are much lower than 1 , indicating that their group means are different.

Yet another statistic (table 4) is the significance test for the equality of group means for each ratio. The $\mathrm{F}$ value is obtained by dividing the between-group variation by within-group variation. The observed significance level (0.0000; table 4) for every $\log _{\mathrm{e}}$-transformed ratio is less than 0.01 (even $<0.0001$ ), the hypothesis $\left(H_{0}\right)$ that all group means are equal is rejected in each case at the confidence level of $99 \%$ (even at the highest confidence level of $99.99 \%$ ). This means that, in theory, each ratio can be considered as a possible candidate for predictor variable in the discriminant analysis.

\subsection{Selection of predictor variables,} number of groups considered simultaneously, discriminant score, and classification rule

The rest of our procedure was similar to the one described in detail by Agrawal et al (2004). The success of discriminant analysis depends on selection of appropriate predictor variables. This selection was based on the test of Wilk's lambda, and these variables were selected by a stepwise method, with minimization of Wilk's lambda as the criterion for variable selection. At each step the variable that resulted in the smallest lambda for the discriminant function was selected.

The use of discriminant functions is advantageous because each discriminant function can represent several of the compositional variables. The discriminant analysis is one of the statistical multivariate techniques most commonly used to distinguish between members of different, pre-defined, groups or classes, on the basis of observations regarding them. The actual aim in mathematical terms is to summarize p-dimensional observations from $g$ classes on a one-dimensional linear function that discriminates between these classes by some measure of maximum separation, and later serves the purpose of classifying samples of unknown classes. An 'unknown' class should be one of the classes that were actually used for the discriminant analysis to begin with. Although the concept of discriminant analysis is simple, more details on linear discriminant analysis, being highly mathematical and complex, are probably beyond the scope of the present paper; these can be consulted in standard books on multivariate techniques such as Morrison (1990) and Reyment and Savazzi (1997). Finally, as in Agrawal et al (2004) we used the SPSS/PC+ statistical package for performing the discriminant analysis.

We performed the discriminant analysis for all combinations of the four groups by taking only four and three groups at a time. The discriminant score $(D)$ of the individual case in each group can be obtained as:

$$
D=B_{1} X_{1}+B_{2} X_{2}+\cdots+B_{p} X_{p}+B_{0},
$$

where $X_{i}$ is the value of the $\log _{\mathrm{e}}$-transformed $i$ th major-element ratio (e.g., the first ratio is $\left.\ln \left(\mathrm{TiO}_{2} / \mathrm{SiO}_{2}\right)_{\text {adj }}\right), \quad B_{i}$ is the $i$ th discriminant 
function coefficient, and $B_{0}$ is a constant. These $p$ coefficients and the constant can have positive or negative values. The discriminant analysis makes use of all or some of the available $\log _{\mathrm{e}}$-transformed major-element ratios $\left(X_{i}^{\prime} \mathrm{s}\right)$ and fixes the best values of $B_{i}$ for each chosen $X_{i}$ to obtain the maximum separation between all groups.

If $g$ groups are involved in discriminant analysis, $g-1$ discriminant functions are obtained. The number of discriminant functions in three- and fourgroup discriminant analyses, therefore, are 2 and 3 respectively. The discriminant scores D1, D2, and D3 were calculated using equation (1). From the discriminant scores, the mean discriminant scores for the groups, known as group centroids, were calculated.

The rule for classifying a given basic rock sample into one of the tectonic groups was obtained from its discriminant score $D$. The probability that a basic rock with a score $D$ belongs to group $i$ is estimated by:

$$
P\left(g_{i} / D\right)=P\left(D / g_{i}\right) P\left(g_{i}\right)
$$

where $P\left(g_{i} / D\right)$ is the posterior probability, $P\left(D / g_{i}\right)$ is the conditional probability, and $P\left(g_{i}\right)$ is the prior probability.

The prior probability - an estimate of the likelihood that a case belongs to a particular group when no information is available - was assumed to be equal for all groups. The conditional probability $P\left(D / g_{i}\right)$ of $D$ for a given group is calculated from the probability of obtaining a particular discriminant score value $D$ if the case is a member of that group, under the assumption that the discriminant scores of the cases used in the analysis are normally distributed and the parameters of the distribution can be estimated from the mean discriminant scores. The case is assumed to belong to a particular group and the probability of the observed score for that particular membership is estimated. From these prior and conditional probabilities, the posterior probability $P\left(g_{i} / D\right)$ is then estimated using Bayes' rule. From its discriminant score $D$, a case is classified in a group for which the posterior probability is largest.

Finally, concerning field boundaries in geochemical discrimination diagrams, Agrawal (1999) argued at length that the practice of defining assignment rules with the least precise and subjective method of eye-fitted lines must be abondoned. He illustrated the proposed statistical method using a simple case of two variables from two classes. We have adopted this approach to draw probability based boundaries in our diagrams.

\section{Results of the discriminant analysis}

Table 5 shows the results of the discriminant analysis of the present database and their comparison with our earlier work (Agrawal et al 2004). In the first column, the rows marked A are for Agrawal et al (2004). The results of the rows $\mathrm{B}$ were obtained in exactly the same manner as in Agrawal et al (2004), i.e., using majorelement concentrations, but from the extended database. Finally, the rows $\mathrm{C}$ correspond to the results using $\log _{\mathrm{e}}$-transformation of the majorelement ratios from exactly the same database as in rows $\mathrm{B}$.

The discriminant analysis was performed five times using the training set: once for all four tectonic groups; and four times for all possible combinations of three groups taken at a time. All ratios were found to be useful as predictor variables under the criterion of reduction of Wilk's lambda for the discriminant function. Then, the assignment rules from the discriminant analysis of the training set were used to classify samples of the testing set, treating them as 'unknown' cases.

The rates of correct classification of the training as well as testing sets have been calculated, and are compared with our earlier work (table 5). The overall \% success rate (rate of correct classification) for discriminant function diagrams proposed by Agrawal et al (2004) varied from $~ 83$ to $93 \%$ (cases $\mathrm{A}$ in table 5 ). The same procedure for the larger dataset resulted in similar \% success rates of $\sim 82$ to $94 \%$ (cases $\mathrm{B}$ in table 5 ). The $\log _{\mathrm{e}}$-transformation procedure, however, provided higher overall \% success rates of $\sim 83$ to 97\% (in all cases $\mathrm{C}$ as compared to the respective cases $\mathrm{B}$ in table 5), suggesting that such a transformation is a statistically-correct procedure and, therefore, should be used in future for compositional data handling. However, small decreases in \% success rates for some individual tectonic groups (6 out of a total of 32 cases; table 5) occur, the reason for which is not clear at present, but might be related to the statistical nature of the multivariate techniques. In this context, we must emphasize that these discriminant function diagrams should be used on this very statistical basis, i.e., by taking into account that the boundaries are statistically-based and a given field at best encloses only a certain percentage of and not all rock samples from a given setting (e.g., for our new diagrams 83 to $97 \%$ of samples used for the training or testing sets). This will be better illustrated in the application section of this paper. 
Table 5. Assessment of correct classification (\%) between the IAB (1), CRB (2), OIB (3), and MORB (4) groups.

\begin{tabular}{|c|c|c|c|c|c|c|c|c|}
\hline Dataset & Group & Set & $n$ & IAB (1) & CRB (2) & OIB (3) & MORB (4) & Overall \\
\hline $\mathrm{A}$ & $1-2-3-4$ & Training & 1059 & 80.8 & 70.3 & 80.6 & 93.0 & 83.3 \\
\hline B & $1-2-3-4$ & Training & 2332 & 81.8 & 70.9 & 77.3 & 93.8 & 82.0 \\
\hline $\mathrm{C}$ & $1-2-3-4$ & Training & 2332 & 90.4 & 77.9 & 76.7 & 94.8 & 85.6 \\
\hline $\mathrm{A}$ & $1-2-3-4$ & Testing & 100 & 84.0 & 80.0 & 88.0 & 92.0 & 86.0 \\
\hline B & $1-2-3-4$ & Testing & 400 & 91.0 & 78.0 & 73.0 & 94.0 & 84.0 \\
\hline $\mathrm{C}$ & $1-2-3-4$ & Testing & 400 & 94.0 & 84.0 & 71.0 & 97.0 & 86.5 \\
\hline $\mathrm{A}$ & $1-2-3$ & Training & 660 & 84.0 & 79.4 & 79.7 & & 85.0 \\
\hline B & $1-2-3$ & Training & 1506 & 92.6 & 77.9 & 79.9 & & 82.1 \\
\hline $\mathrm{C}$ & $1-2-3$ & Training & 1506 & 91.2 & 80.0 & 85.0 & & 84.3 \\
\hline $\mathrm{A}$ & $1-2-3$ & Testing & 75 & 92.0 & 88.0 & 84.0 & & 85.0 \\
\hline B & $1-2-3$ & Testing & 300 & 94.0 & 78.0 & 79.0 & & 83.7 \\
\hline $\mathrm{C}$ & $1-2-3$ & Testing & 300 & 94.0 & 83.0 & 83.0 & & 86.7 \\
\hline $\mathrm{A}$ & $1-2-4$ & Training & 832 & 80.8 & 84.7 & & 93.5 & 87.9 \\
\hline B & $1-2-4$ & Training & 2332 & 84.8 & 84.2 & & 94.1 & 88.7 \\
\hline $\mathrm{C}$ & $1-2-4$ & Training & 2332 & 91.2 & 91.4 & & 97.6 & 94.1 \\
\hline $\mathrm{A}$ & $1-2-4$ & Testing & 75 & 88.0 & 96.0 & & 92.0 & 92.0 \\
\hline B & $1-2-4$ & Testing & 400 & 92.0 & 94.0 & & 95.0 & 93.7 \\
\hline $\mathrm{C}$ & $1-2-4$ & Testing & 400 & 94.0 & 97.0 & & 99.0 & 96.7 \\
\hline $\mathrm{A}$ & $1-3-4$ & Training & 850 & 84.8 & & 95.6 & 94.5 & 92.2 \\
\hline B & $1-3-4$ & Training & 1661 & 86.8 & & 85.2 & 96.2 & 91.0 \\
\hline $\mathrm{C}$ & $1-3-4$ & Training & 1661 & 95.6 & & 87.3 & 96.7 & 93.8 \\
\hline A & $1-3-4$ & Testing & 75 & 92.0 & & 96.0 & 92.0 & 93.3 \\
\hline B & $1-3-4$ & Testing & 300 & 93.0 & & 82.0 & 98.0 & 91.0 \\
\hline $\mathrm{C}$ & $1-3-4$ & Testing & 300 & 99.0 & & 84.0 & 100.0 & 94.3 \\
\hline A & $2-3-4$ & Training & 835 & & 71.8 & 82.4 & 97.5 & 86.9 \\
\hline B & $2-3-4$ & Training & 1969 & & 76.6 & 78.0 & 98.4 & 86.1 \\
\hline $\mathrm{C}$ & $2-3-4$ & Training & 1969 & & 81.1 & 76.9 & 96.0 & 86.3 \\
\hline A & $2-3-4$ & Testing & 75 & & 76.0 & 88.0 & 96.0 & 86.7 \\
\hline B & $2-3-4$ & Testing & 300 & & 79.0 & 72.0 & 98.0 & 83.0 \\
\hline $\mathrm{C}$ & $2-3-4$ & Testing & 300 & & 83.0 & 71.0 & 99.0 & 84.7 \\
\hline
\end{tabular}

$\mathbf{A}=$ results from Agrawal et al (2004); $\mathbf{B}=$ new results with larger dataset using raw data, and $\mathbf{C}=$ new results with larger dataset using $\log _{\mathrm{e}}$ transformation of ratios.

\subsection{Discrimination between $I A B-C R B-O I B-M O R B$ groups}

As in Agrawal et al (2004), we developed five new discriminant function diagrams for the four tectonic types of basic (and ultrabasic) rocks (IAB, CRB, OIB, and MORB). Figure 5 includes all four tectonic types of basic (and ultrabasic) rocks, whereas each of the four diagrams in figure $6(\mathrm{a}-\mathrm{d})$ presents samples from three groups. Thus, each tectonic group appears in four of these five diagrams. Each diagram is a plot of two discriminant functions, DF1 and DF2, respectively in $\mathrm{x}$ - and y-axes. The discriminant functions reduce the dimensionality of the problem, in this case (figure 5) from 10 major-element ratio $\log _{\mathrm{e}}$-transformed variables to two dimensions of discriminant functions DF1 and DF2. In this four-group discriminant analysis, which resulted in figure 5 , three discriminant functions were obtained, but the first two functions accounted for $94.16 \%$ of between-group variability (table 6 ), the third function being relatively insignificant (capable of explaining the remaining $5.84 \%$ of this variability). For three-group diagrams, the between-group variability is completely represented by two discriminant functions (table 7).

The classification rules in figure 5 are based on only two discriminant functions, DF1 and DF2 as follows (note the coefficients or the multiplication factors for each $\log _{\mathrm{e}}$-transformed ratio after rounding to four decimal places are listed in table 6):

$$
\begin{aligned}
\mathrm{DF} 1= & -4.6761 \cdot \ln \left(\mathrm{TiO}_{2} / \mathrm{SiO}_{2}\right)_{\mathrm{adj}} \\
& +2.5330 \cdot \ln \left(\mathrm{Al}_{2} \mathrm{O}_{3} / \mathrm{SiO}_{2}\right)_{\mathrm{adj}} \\
& -0.3884 \cdot \ln \left(\mathrm{Fe}_{2} \mathrm{O}_{3} / \mathrm{SiO}_{2}\right)_{\mathrm{adj}} \\
& +3.9688 \cdot \ln \left(\mathrm{FeO} / \mathrm{SiO}_{2}\right)_{\mathrm{adj}} \\
& +0.8980 \cdot \ln \left(\mathrm{MnO} / \mathrm{SiO}_{2}\right)_{\mathrm{adj}} \\
& -0.5832 \cdot \ln \left(\mathrm{MgO} / \mathrm{SiO}_{2}\right)_{\mathrm{adj}}
\end{aligned}
$$




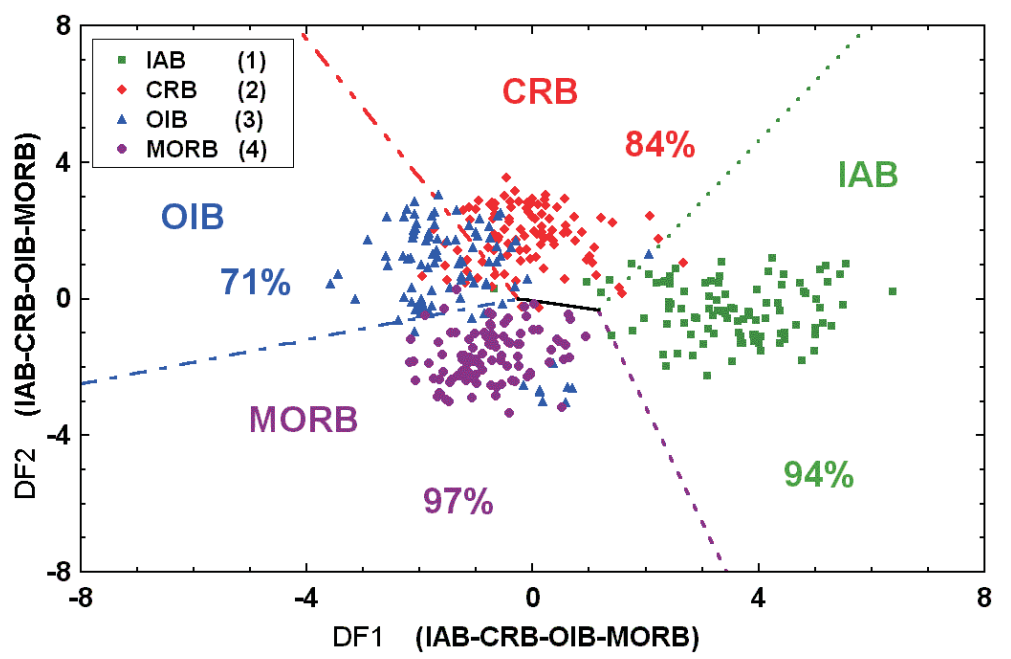

Figure 5. Diagram IAB-CRB-OIB-MORB (1-2-3-4) displaying samples of the testing set. The symbols are shown as inset. The coordinates (DF1, DF2) of the field boundaries are as follows: $(1.160,-0.333)$ and $(5.912,8.000)$ for the boundary IAB-CRB; $(-0.266,0.020)$ and $(-4.190,8.000)$ for CRB-OIB; $(-8.000,-2.490)$ and $(-0.266,0.020)$ for OIB-MORB; $(1.160,-0.333)$ and $(3.431,-8.000)$ for IAB-MORB; and $(1.160,-0.333)$ and $(-0.266,0.020)$ for CRB-MORB. The percentages shown in this figure (IAB 94\%, CRB 84\%, OIB 71\%, and MORB 97\%) refer to the percentage of the correct classification for samples of the testing set (see table 5). Discriminant function coefficients are displayed in table 6; i.e., for computing the discriminant functions DF1 and DF2 for 'unknown' samples use equations (3) and (4) in the text.
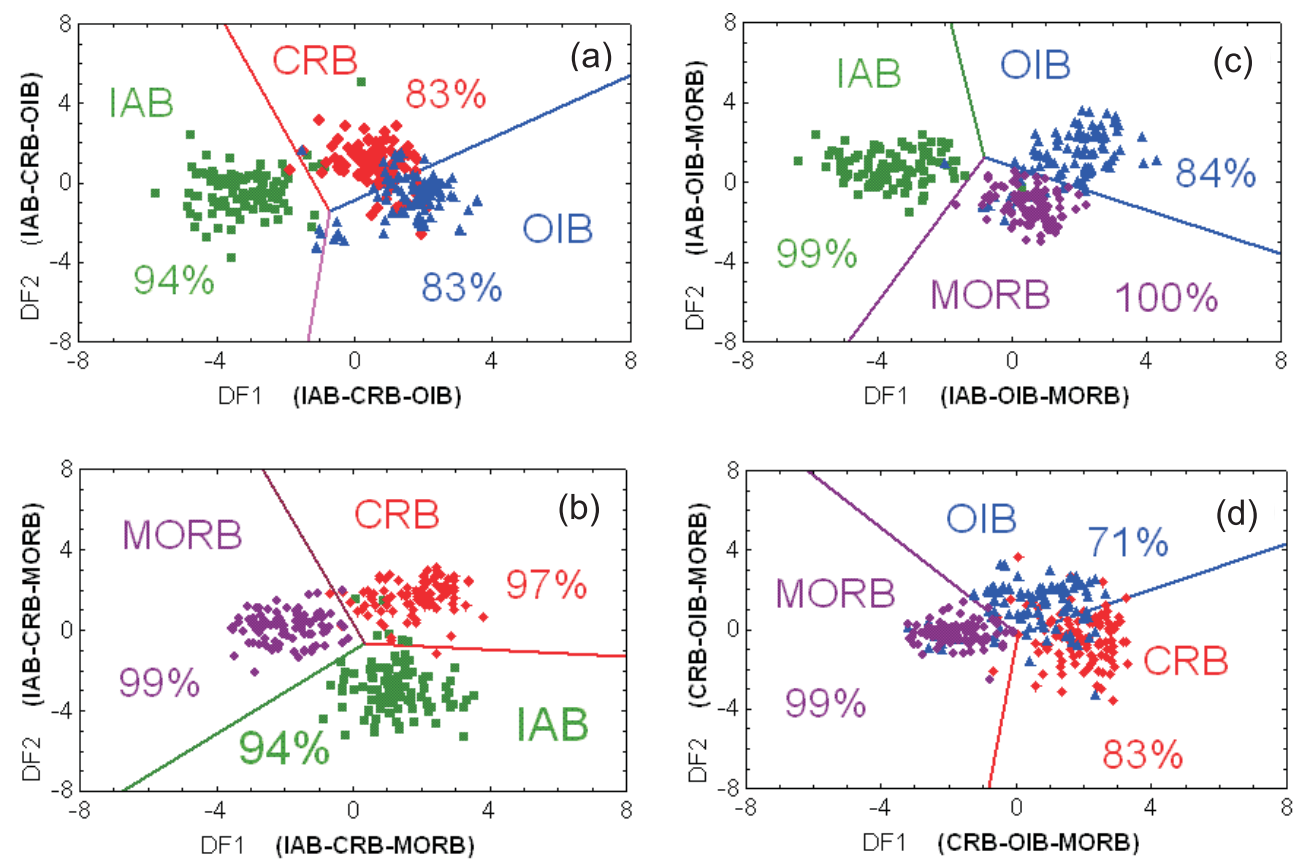

Figure 6. Three-groups at a time diagrams displaying samples of the testing set. The symbols are shown as inset. The percentages shown in this figure (e.g., IAB 94\%, CRB $83 \%$, and OIB $83 \%$ for the first diagram) refer to the percentage of the correct classification for samples of the testing set (see table 6). Discriminant function coefficients are displayed in table 6; i.e., for computing the discriminant functions DF1 and DF2 for 'unknown' samples use them analogously to construct new equations similar to equations (3) and (4) used for four groups. (a) Group IAB-CRB-OIB $(1-2-3)$ : $(-0.733,-1.405)$ and $(-3.788,8.000)$ for the boundary IAB-CRB; $(-0.733,-1.405)$ and $(8.000,5.428)$ for CRB-OIB; and $(-1.343,-8.000)$ and $(-0.733,-1.405)$ for IAB-OIB. (b) Group IAB-CRB-MORB $(1-2-4)$ : $(8.000,-1.332)$ and $(0.361,-0.619)$ for the boundary IAB-CRB; $(-2.673,8.000)$ and $(0.361,-0.619)$ for CRB-MORB; and $(-6.779,-8.000)$ and $(0.361,-0.619)$ for IAB-MORB. (c) Group IAB-OIB-MORB $(1-3-4):(-0.830,1.224)$ and $(-1.824,8.000)$ for the boundary IAB-OIB; $(-0.830,1.224)$ and $(8.000,-3.583)$ for OIB-MORB; and $(-4.865,-8.000)$ and $(-0.830,1.224)$ for IAB-MORB. (d) Group CRB-OIB-MORB $(2-3-4):(0.029,-0.222)$ and $(8.000,4.322)$ for the boundary CRB-OIB; $(0.029,-0.222)$ and $(-6.177$, $8.000)$ for OIB-MORB; and $(-0.819,-8.000)$ and $(0.029,-0.222)$ for CRB-MORB. 
Table 6. The discriminant function coefficients for the four group sets $I A B-C R B-O I B-M O R B$.

\begin{tabular}{lrr}
\hline $\begin{array}{l}\mathrm{Log}_{\mathrm{e}} \text {-transformed } \\
\text { ratio variable }\end{array}$ & \multicolumn{1}{c}{$\mathrm{DF} 1$} & \multicolumn{1}{c}{ DF2 } \\
\hline $\ln \left(\mathrm{TiO}_{2} / \mathrm{SiO}_{2}\right)_{\text {adj }}$ & -4.6761 & 0.6751 \\
$\ln \left(\mathrm{Al}_{2} \mathrm{O}_{3} / \mathrm{SiO}_{2}\right)_{\text {adj }}$ & 2.5330 & 4.5895 \\
$\ln \left(\mathrm{Fe}_{2} \mathrm{O}_{3} / \mathrm{SiO}_{2}\right)_{\text {adj }}$ & -0.3884 & 2.0897 \\
$\ln \left(\mathrm{FeO} / \mathrm{SiO}_{2}\right)_{\text {adj }}$ & 3.9688 & 0.8514 \\
$\ln \left(\mathrm{MnO}_{2} / \mathrm{SiO}_{2}\right)_{\text {adj }}$ & 0.8980 & -0.4334 \\
$\ln \left(\mathrm{MgO}_{2} / \mathrm{SiO}_{2}\right)_{\text {adj }}$ & -0.5832 & 1.4832 \\
$\ln \left(\mathrm{CaO}_{2} / \mathrm{SiO}_{2}\right)_{\text {adj }}$ & -0.2896 & -2.3627 \\
$\ln \left(\mathrm{Na}_{2} \mathrm{O} / \mathrm{SiO}_{2}\right)_{\text {adj }}$ & -0.2704 & -1.6558 \\
$\ln \left(\mathrm{K}_{2} \mathrm{O} / \mathrm{SiO}_{2}\right)_{\text {adj }}$ & 1.0810 & 0.6757 \\
$\ln \left(\mathrm{P}_{2} \mathrm{O}_{5} / \mathrm{SiO}_{2}\right)_{\text {adj }}$ & 0.1845 & 0.4130 \\
$\mathrm{Constant}^{\text {Cof variance }}$ & 1.5445 & 13.1639 \\
\hline $\mathrm{T}$ & 55.15 & 39.01 \\
\hline
\end{tabular}

The third function DF3 obtained in this analysis accounted for the remaining $5.84 \%$ between-groups variance.

$$
\begin{aligned}
& -0.2896 \cdot \ln \left(\mathrm{CaO} / \mathrm{SiO}_{2}\right)_{\text {adj }} \\
& -0.2704 \cdot \ln \left(\mathrm{Na}_{2} \mathrm{O} / \mathrm{SiO}_{2}\right)_{\text {adj }} \\
& +1.0810 \cdot \ln \left(\mathrm{K}_{2} \mathrm{O} / \mathrm{SiO}_{2}\right)_{\text {adj }} \\
& +0.1845 \cdot \ln \left(\mathrm{P}_{2} \mathrm{O}_{5} / \mathrm{SiO}_{2}\right)_{\text {adj }}+1.5445
\end{aligned}
$$

\begin{tabular}{|c|c|c|c|c|c|}
\hline \multicolumn{3}{|c|}{ IAB-CRB-OIB (1-2-3) } & \multicolumn{3}{|c|}{ IAB-CRB-MORB (1-2-4) } \\
\hline $\begin{array}{l}\log _{\mathrm{e}} \text {-transformed } \\
\text { ratio variable }\end{array}$ & DF1 & DF2 & $\begin{array}{l}\log _{e} \text {-transformed } \\
\text { ratio variable }\end{array}$ & DF1 & DF2 \\
\hline $\ln \left(\mathrm{TiO}_{2} / \mathrm{SiO}_{2}\right)_{\mathrm{adj}}$ & 3.9998 & -1.3705 & $\ln \left(\mathrm{TiO}_{2} / \mathrm{SiO}_{2}\right)_{\mathrm{adj}}$ & -1.5736 & 3.9844 \\
\hline $\ln \left(\mathrm{Al}_{2} \mathrm{O}_{3} / \mathrm{SiO}_{2}\right)_{\mathrm{adj}}$ & -2.2385 & 3.0104 & $\ln \left(\mathrm{Al}_{2} \mathrm{O}_{3} / \mathrm{SiO}_{2}\right)_{\text {adj }}$ & 6.1498 & 0.2200 \\
\hline $\ln \left(\mathrm{Fe}_{2} \mathrm{O}_{3} / \mathrm{SiO}_{2}\right)_{\mathrm{adj}}$ & 0.8110 & 0.3239 & $\ln \left(\mathrm{Fe}_{2} \mathrm{O}_{3} / \mathrm{SiO}_{2}\right)_{\mathrm{adj}}$ & 1.5544 & 1.1516 \\
\hline $\ln \left(\mathrm{FeO} / \mathrm{SiO}_{2}\right)_{\mathrm{adj}}$ & -2.5865 & 1.8998 & $\ln \left(\mathrm{FeO} / \mathrm{SiO}_{2}\right)_{\mathrm{adj}}$ & 3.4134 & -2.2036 \\
\hline $\ln \left(\mathrm{MnO} / \mathrm{SiO}_{2}\right)_{\mathrm{adj}}$ & -1.2433 & -1.9746 & $\ln \left(\mathrm{MnO} / \mathrm{SiO}_{2}\right)_{\mathrm{adj}}$ & -0.0087 & -1.6228 \\
\hline $\ln \left(\mathrm{MgO} / \mathrm{SiO}_{2}\right)_{\mathrm{adj}}$ & 0.4872 & 1.4411 & $\ln \left(\mathrm{MgO} / \mathrm{SiO}_{2}\right)_{\mathrm{adj}}$ & 1.2480 & 1.4291 \\
\hline $\ln \left(\mathrm{CaO} / \mathrm{SiO}_{2}\right)_{\mathrm{adj}}$ & -0.3153 & -2.2656 & $\ln \left(\mathrm{CaO} / \mathrm{SiO}_{2}\right)_{\mathrm{adj}}$ & -2.1103 & -1.2524 \\
\hline $\ln \left(\mathrm{Na}_{2} \mathrm{O} / \mathrm{SiO}_{2}\right)_{\mathrm{adj}}$ & 0.4325 & 1.8665 & $\ln \left(\mathrm{Na}_{2} \mathrm{O} / \mathrm{SiO}_{2}\right)_{\mathrm{adj}}$ & -0.7576 & 0.3581 \\
\hline $\ln \left(\mathrm{K}_{2} \mathrm{O} / \mathrm{SiO}_{2}\right)_{\mathrm{adj}}$ & -1.0262 & 0.2872 & $\ln \left(\mathrm{K}_{2} \mathrm{O} / \mathrm{SiO}_{2}\right)_{\mathrm{adj}}$ & 1.1431 & -0.6414 \\
\hline $\ln \left(\mathrm{P}_{2} \mathrm{O}_{5} / \mathrm{SiO}_{2}\right)_{\mathrm{adj}}$ & 0.0514 & 0.8138 & $\ln \left(\mathrm{P}_{2} \mathrm{O}_{5} / \mathrm{SiO}_{2}\right)_{\mathrm{adj}}$ & 0.3524 & 0.2646 \\
\hline Constant & -0.5718 & 1.8202 & Constant & 16.8712 & 5.0506 \\
\hline \multicolumn{3}{|c|}{ IAB-OIB-MORB (1-3-4) } & \multicolumn{3}{|c|}{ CRB-OIB-MORB (2-3-4) } \\
\hline $\begin{array}{l}\log _{\mathrm{e}} \text {-transformed } \\
\text { ratio variable }\end{array}$ & DF1 & DF2 & $\begin{array}{l}\log _{\mathrm{e}} \text {-transformed } \\
\text { ratio variable }\end{array}$ & DF1 & DF2 \\
\hline $\ln \left(\mathrm{TiO}_{2} / \mathrm{SiO}_{2}\right)_{\mathrm{adj}}$ & 5.3396 & 1.1799 & $\ln \left(\mathrm{TiO}_{2} / \mathrm{SiO}_{2}\right)_{\text {adj }}$ & -0.5183 & 5.0509 \\
\hline $\ln \left(\mathrm{Al}_{2} \mathrm{O}_{3} / \mathrm{SiO}_{2}\right)_{\mathrm{adj}}$ & -1.6279 & 5.5114 & $\ln \left(\mathrm{Al}_{2} \mathrm{O}_{3} / \mathrm{SiO}_{2}\right)_{\text {adj }}$ & 4.9886 & -0.4972 \\
\hline $\ln \left(\mathrm{Fe}_{2} \mathrm{O}_{3} / \mathrm{SiO}_{2}\right)_{\mathrm{adj}}$ & 0.8338 & 2.7737 & $\ln \left(\mathrm{Fe}_{2} \mathrm{O}_{3} / \mathrm{SiO}_{2}\right)_{\mathrm{adj}}$ & 2.2204 & 1.0046 \\
\hline $\ln \left(\mathrm{FeO} / \mathrm{SiO}_{2}\right)_{\mathrm{adj}}$ & -4.7362 & -0.1341 & $\ln \left(\mathrm{FeO} / \mathrm{SiO}_{2}\right)_{\mathrm{adj}}$ & 1.1801 & -3.3848 \\
\hline $\ln \left(\mathrm{MnO} / \mathrm{SiO}_{2}\right)_{\text {adj }}$ & -0.1254 & 0.6672 & $\ln \left(\mathrm{MnO} / \mathrm{SiO}_{2}\right)_{\mathrm{adj}}$ & -0.3008 & 0.5528 \\
\hline $\ln \left(\mathrm{MgO} / \mathrm{SiO}_{2}\right)_{\text {adj }}$ & 0.6452 & 1.1045 & $\ln \left(\mathrm{MgO} / \mathrm{SiO}_{2}\right)_{\mathrm{adj}}$ & 1.3297 & 0.2925 \\
\hline $\ln \left(\mathrm{CaO} / \mathrm{SiO}_{2}\right)_{\mathrm{adj}}$ & 1.5153 & -1.7231 & $\ln \left(\mathrm{CaO} / \mathrm{SiO}_{2}\right)_{\mathrm{adj}}$ & -2.1834 & 0.4007 \\
\hline $\ln \left(\mathrm{Na}_{2} \mathrm{O} / \mathrm{SiO}_{2}\right)_{\mathrm{adj}}$ & -0.8154 & -3.8948 & $\ln \left(\mathrm{Na}_{2} \mathrm{O} / \mathrm{SiO}_{2}\right)_{\mathrm{adj}}$ & -1.9319 & -2.8637 \\
\hline $\ln \left(\mathrm{K}_{2} \mathrm{O} / \mathrm{SiO}_{2}\right)_{\mathrm{adj}}$ & -0.8888 & 0.9471 & $\ln \left(\mathrm{K}_{2} \mathrm{O} / \mathrm{SiO}_{2}\right)_{\mathrm{adj}}$ & 0.6976 & -0.2189 \\
\hline $\ln \left(\mathrm{P}_{2} \mathrm{O}_{5} / \mathrm{SiO}_{2}\right)_{\mathrm{adj}}$ & -0.2255 & -0.1082 & $\ln \left(\mathrm{P}_{2} \mathrm{O}_{5} / \mathrm{SiO}_{2}\right)_{\mathrm{adj}}$ & 0.8998 & -1.0558 \\
\hline Constant & 5.7755 & 15.4984 & Constant & 13.2625 & 2.8877 \\
\hline
\end{tabular}

$\mathrm{DF} 2=0.6751 \cdot \ln \left(\mathrm{TiO}_{2} / \mathrm{SiO}_{2}\right)_{\mathrm{adj}}$

$$
\begin{aligned}
& +4.5895 \cdot \ln \left(\mathrm{Al}_{2} \mathrm{O}_{3} / \mathrm{SiO}_{2}\right)_{\text {adj }} \\
& +2.0897 \cdot \ln \left(\mathrm{Fe}_{2} \mathrm{O}_{3} / \mathrm{SiO}_{2}\right)_{\text {adj }} \\
& +0.8514 \cdot \ln \left(\mathrm{FeO} / \mathrm{SiO}_{2}\right)_{\text {adj }} \\
& -0.4334 \cdot \ln \left(\mathrm{MnO} / \mathrm{SiO}_{2}\right)_{\text {adj }} \\
& +1.4832 \cdot \ln \left(\mathrm{MgO} / \mathrm{SiO}_{2}\right)_{\text {adj }} \\
& -2.3627 \cdot \ln \left(\mathrm{CaO} / \mathrm{SiO}_{2}\right)_{\text {adj }} \\
& -1.6558 \cdot \ln \left(\mathrm{Na}_{2} \mathrm{O} / \mathrm{SiO}_{2}\right)_{\text {adj }}
\end{aligned}
$$

Table 7. The discriminant function coefficients for the three group discriminant analyses.

The two functions (DF1 and DF2) together account for $100 \%$ of between-group variance in all the above three-group analyses. 


$$
\begin{aligned}
& +0.6757 \cdot \ln \left(\mathrm{K}_{2} \mathrm{O} / \mathrm{SiO}_{2}\right)_{\text {adj }} \\
& +0.4130 \cdot \ln \left(\mathrm{P}_{2} \mathrm{O}_{5} / \mathrm{SiO}_{2}\right)_{\text {adj }}+13.1639
\end{aligned}
$$

Similarly, the discriminant function coefficients for the four diagrams of the three-group analyses (figure $6 \mathrm{a}-\mathrm{d}$ ) are given in table 7 . Thus, the relevant functions can be easily constructed following the methodology of equations (3) and (4). In this study, all major-element ratios are involved in each of the five diagrams, whereas in our earlier discriminant analysis (Agrawal et al 2004) of raw data (majorelement concentrations), the use of different majorelements (not all the elements under consideration) in a given diagram had resulted in minimization of the Wilk's lambda for the discriminant function.

Further, in figures 5 and $6(\mathrm{a}-\mathrm{d})$, the line separating any two groups represents values of scores DF1 and DF2 that would yield equal posterior probabilities for the two groups, while the posterior probabilities for the other group(s) would be zero, except at the triple junction (point of intersection of three groups) where this probability belonging to the three groups is equal, i.e., $\sim 33.33 \%$. As we move away from a field boundary into the field of a group, the posterior probability becomes highest for that group. The boundary lines in each diagram (figures 5 and $6 \mathrm{a}-\mathrm{d}$ ) are explicitly specified with their coordinates, which should facilitate their reproduction in diverse applications.

\subsection{Comparison of linear discriminant analysis with classification trees}

Recently, Vermeesch (2006) presented the use of classification trees for tectonic discrimination of basalts from three tectonic settings (IAB, OIB, and MORB) and opined that this method provides a better performance (higher success rates) in comparison with linear discriminant analysis. We consider this claim to be false because Vermeesch (2006) ignored the previously published discriminant analysis work by Agrawal et al (2004) who obtained very high success rates of 76 to $96 \%$ for the discrimination of even four (not just three) tectonic settings (IAB, CRB, OIB, and MORB). Further, the success rates obtained in our present work by using log-ratio transformations are even higher (82 to $97 \%$ ) than those obtained without such transformations (76 to $96 \%$ ), whereas Vermeesch (2006) reported only $84 \%$ and $89 \%$ success using two sets of classification trees. Therefore, at least in terms of the 'success rate' parameter the linear discriminant analysis is found to perform equally well, if not better than the method of classification trees. The concern that geochemical discriminant diagrams are often based on mean values of many analyses of a given area is also not relevant because
Agrawal et al (2004) did not use such a procedure, nor this has been done in our present work. Finally, Vermeesch (2006) also expressed concerns about the fact that the decision boundaries of most tectonic discrimination diagrams are drawn by eye. This is true for the references that Vermeesch cited, but certainly not so for the entire published literature on linear discriminant analysis because, as has been expressed in our paper, Agrawal (1999) and Agrawal et al (2004) used an objective statisticallybased approach for drawing these boundaries. The same is the case of our present work. Evaluation of other so-called 'advantages' of classification trees pointed out by Vermeesch (2006) should await the completion of our future plans (see section 5.7 below).

\subsection{Considerations on mis-classified samples in discrimination diagrams}

Analytical errors inevitably present in all geochemical data constitute one major source of misclassifications, especially for samples falling in a 'wrong' field at locations close to the field boundaries in figures 5 and $6(\mathrm{a}-\mathrm{d})$. We hope that the international community establishes some basic rules to be followed for obtaining and reporting geochemical data along with the respective individual uncertainties, as has been attempted recently by Guevara et al (2005) for X-ray flourescence data and Santoyo et al (2006) for high-performance liquid chromatography data. This will help us not only in the better proposal and use of the discrimination diagrams but also for numerous other applications such as in geological process identification and modelling of magma sources. Actual effects of these errors in linear discriminant analysis will be difficult to evaluate; however, reporting of these individual errors will help us to establish a more reliable database than has been possible at present. In any case, the individual errors can be taken into account (using error propagation theory; see Verma and Santoyo 1997; Bevington and Robinson 2003; Verma 2000b, 2005; García-Valladares et al 2006; Verma et al 2006) during the application stage of our discriminant function diagrams; this will be illustrated in a subsequent paper. The total propagated errors in the final data points in a given discriminant function diagram will depend in a complex manner on the individual errors of all 'ratioed' elements (e.g., errors of $\mathrm{TiO}_{2}$ and $\mathrm{SiO}_{2}$ for $\left.\ln \left(\mathrm{TiO}_{2} / \mathrm{SiO}_{2}\right)_{\text {adj }}\right)$ as well as the magnitude of the respective discriminant function coefficients (see equations (3) and (4) above and tables 5-7 for these coefficients for different diagrams and the error propagation theory).

A synthesis of mis-classified samples from both the training and testing sets is presented in table 8 . 
Table 8. Synthesis of mis-classified samples from training and testing sets.

\begin{tabular}{|c|c|c|c|c|c|c|c|c|c|c|c|}
\hline \multirow{2}{*}{$\begin{array}{c}\text { Using } \\
\text { figure \# }\end{array}$} & \multicolumn{6}{|c|}{ Training set } & \multicolumn{5}{|c|}{ Testing set } \\
\hline & Group & IAB & CRB & OIB & MORB & Total & IAB & CRB & OIB & MORB & Total \\
\hline \multirow{4}{*}{5} & (IAB & - & 24 & 1 & 10 & 35 & - & 6 & 0 & 0 & 6 \\
\hline & CRB & 19 & - & 107 & 22 & 148 & 1 & - & 15 & 0 & 16 \\
\hline & OIB & 2 & 58 & - & 50 & 110 & 0 & 16 & - & 13 & 29 \\
\hline & MORB & 7 & 10 & 26 & - & 43 & 0 & 1 & 2 & - & 3 \\
\hline \multirow{3}{*}{$6 \mathrm{~A}$} & IAB & - & 25 & 7 & n.a. & 32 & - & 6 & 0 & n.a. & 6 \\
\hline & $\{\mathrm{CRB}$ & 20 & - & 114 & n.a. & 134 & 1 & - & 16 & n.a. & 17 \\
\hline & OIB & 11 & 60 & - & n.a. & 71 & 3 & 14 & - & n.a. & 17 \\
\hline \multirow{3}{*}{$6 \mathrm{~B}$} & ( IAB & - & 21 & n.a. & 11 & 32 & - & 6 & n.a. & 0 & 6 \\
\hline & CRB & 21 & - & n.a. & 37 & 58 & 1 & - & n.a. & 2 & 3 \\
\hline & MORB & 8 & 12 & n.a. & - & 20 & 0 & 1 & n.a. & - & 1 \\
\hline \multirow{3}{*}{$6 \mathrm{C}$} & IAB & - & n.a. & 6 & 10 & 16 & - & n.a. & 0 & 1 & 1 \\
\hline & OIB & 6 & n.a. & - & 54 & 60 & 1 & n.a. & - & 15 & 16 \\
\hline & MORB & 7 & n.a. & 20 & - & 27 & 0 & n.a. & 0 & - & 0 \\
\hline \multirow{3}{*}{$6 \mathrm{D}$} & ( CRB & n.a. & - & 100 & 25 & 125 & n.a. & - & 16 & 1 & 17 \\
\hline & OIB & n.a. & 58 & - & 51 & 109 & n.a. & 15 & - & 13 & 28 \\
\hline & MORB & n.a. & 14 & 19 & - & 33 & n.a. & 0 & 1 & - & 1 \\
\hline
\end{tabular}

Total number of samples in the training set: IAB (363 samples), CRB (671 samples), OIB (472 samples), and MORB (826 samples); and in the testing set: IAB (100 samples), CRB (100 samples), OIB (100 samples), and MORB (100 samples). n.a. not applicable for this diagram; - not applicable for this setting (i.e., the samples in this place are, in fact, correctly classified).

In fact, the mis-classification represents the complementary part of the \% success $(\%$ correct classification) listed in table 5. For example, the 35 mis-classified samples from the IAB training set (table 8) in the four-group discriminant function diagram represent about $9.6 \%$ of all samples used in constructing it with the consequent $\%$ success of $90.4 \%$ (table 5 ). We will provide a detailed analysis of the mis-classified samples only for the four-group diagram (figure 5) because similar reasoning is valid for the three-group diagrams (figure $6 \mathrm{a}-\mathrm{d}$ ).

The mis-classified rocks from the IAB setting (a total of 41 samples; table 8) plot mainly in the field of CRB, viz., 30 samples (24 from the training and 6 from the testing set), followed by 10 samples from the training set mis-classified as MORB. Of these, most of the samples (a total of 20) mis-classified as CRB are from the Ueno basalt province in Japan, for which the original authors (Kimura et al 2002) have stressed the chemical diversity and a complex origin of these rocks. This area is located close to the intersection of three plates - North American plate, Eurasian plate, and Phillipine Sea plate, and is confined within four active faults. The depth of subducting slab surface below the Ueno basalt province varies between 200 and $300 \mathrm{~km}$ (and not the 100 to $200 \mathrm{~km}$ for a typical arc front). For these reasons, the Ueno basalt province area probably does not represent a typical IAB setting (at most a back-arc or a complex setting), with the consequent mis-classification of a large number of IAB samples from our database. Concerning the mis-classified IAB samples as MORB, 5 out of 10 such samples are from the Tonga-Kermedac arc, from which a total of 25 samples were compiled in our database, and therefore, these mis-classified samples represent only a relatively small proportion (20\%) of samples from this arc.

A large number of CRB samples were misclassified as OIB (107 from the training set and 15 from the testing set; table 8). This is probably mainly due to the similarities of mantle sources beneath these two distinct tectonic settings. Numerous samples from CRB setting in Africa (51 samples from Central Afar and Ethiopia rifts, 28 from East Africa rift, 4 from Kenya rift, and 4 from Gregory rift) are mis-classified as OIB. Relatively few (20) CRB samples were misclassified as IAB; most of them come from the western U.S.A. (e.g., 6 from the Basin and Range, 3 from the Colorado Plateau, and 2 from the Rio Grande rift). In fact, all 14 samples compiled in our database as from the Central Afar region of the Ethiopia rift are mis-classified: 9 as OIB and 5 as MORB. For the Ethiopia rift, the mis-classification represents a large percentage $(\sim 60 \%)$ of the compiled samples. For other CRB areas from Africa such as the Kenya and Gregory rifts, the misclassified samples represent a similarly large proportion of the compiled samples. This suggests that beneath Africa the magma sources and processes are similar, at least in terms of major-elements, 
to those for the ocean-island than for the continental rift setting. For other CRB areas, however, the mis-classification is much less significant; for example, for the Massif Central it is about 20\%, and for China and the western USA, it is even less important.

The similarities of mantle sources for OIB and CRB may also account for a large number (74) of OIB samples (58 from the training and 16 from the testing set; table 8) mis-classified as CRB. These are mainly from French Polynesia (26 samples), Hawaiian Islands (22 samples), Socorro Island (7 samples), and Austral Chain $(7$ samples). Samples from the OIB setting mis-classified as MORB mainly come from Iceland (38 samples), Hawaiian Islands (11 samples), Galápagos Islands (6 samples), and St. Paul Island (5 samples). However, for French Polynesia, Hawaiian Islands, and Austral Chain, the mis-classified samples represent only a relatively small percentage $(\sim 15-20 \%)$, whereas for Iceland, Socorro, St. Paul, and Galápagos, a very large percentage of samples are mis-classified, being $73 \%, 50 \%, 45 \%$, and $40 \%$ respectively. The proximity of these islands to an active spreading center (mid-ocean ridge system) may probably explain the higher rate of mis-classification of samples from these OIB areas (for Iceland, Socorro, and Galápagos, see figure 3; for St. Paul, figure 2).

Finally, MORB samples are mis-classified (table 8) mainly as OIB (28 samples) and CRB (11 samples), but represent a very small percentage of the total number of samples (926 samples; table 2) compiled as training and testing sets. Most of these mis-classified samples (28 as OIB and 9 as CRB) come from the North Chile ridge and are MORB samples with very unusual, HFSE-depleted chemistry as documented by the original authors in their paper (Bach et al 1996).

\section{Application of new discriminant function diagrams}

We present a series of case histories or applications, including an example, with an excel template, of these new diagrams for inferring the tectonic setting of tectonically complex areas of Mexico and of older rocks from India. The locations of the application areas are listed in table 9, and also included in figures 1,3 , and 4 . Note that none of the data used here as examples constituted the database for constructing or testing of these diagrams, as confirmed by the respective sample locations (see 'Application' locations in figures 1, 2 and 4$)$.

\subsection{Excel template using a specific example of southern Mexico}

First of all, we will present an excel template highlighting its use with the major-element data for only a few samples from southern Mexico (Verma 2002; these data are available as table DR1 'new data' from the GSA 'data repository' item 2002129; www.geosociety.org/pubs/ft2002.htm). This template will be available from the internet address of the 'Journal of Earth System Science', or directly on request from the first author (SPV) of this paper. The calculations are purposely presented in several steps to make the reader familiar with the type of numbers to be encountered at different stages of calculations because he/she is generally accustomed to looking at the actual concentrations or at best some ratios.

First, the SINCLAS computer program (Verma et al 2002; the computer code can be downloaded from http://www.iamg.org/CGEditor/index.htm; note the internet address is corrected here - it was wrongly printed by the journal; or can be obtained from the first author) is used to obtain the $\mathrm{Fe}_{2} \mathrm{O}_{3}$ and $\mathrm{FeO}$ calculations under the Middlemost option and $100 \%$ adjustment on an anhydrous basis, as required for the correct use of our diagrams.

The Excel file 'SINCLAS_Verma2002InitialData' in the 'Excel Template' folder presents the manner the actual data should be input using the above-mentioned data for 14 samples as example. This file is then processed using the SINCLAS computer program (Verma et al 2002), declaring the file 'SINCLAS_Verma2002ProcessedData' in the 'Excel Template' folder as the output file. Note the 'MIDDLEMO' entry in the 'FE_PROC' column of this file is automatically written by the SINCLAS program when the 'Middlemost' option is chosen. This procedure can be easily repeated for processing other 'unknown' samples.

Then, we present the Excel file 'Discrim Diagrams_template_March2006' in the same file folder as the main Excel template.

The processed data for the samples under study (from the column SIO2ADJ to the column P2O5ADJ, along with the sample names) are manually transferred from the 'SINCLAS_ Verma2002ProcessedData' file on the first part of the 'DataCalc' sheet of the 'Discrim Diagrams_template March 2006' file, as has been done here for the 14 samples from this example (see table 9 for sample locations). The layout of this sheet is such that any number of samples can be easily processed and pasted. Note once again the adj subscript to remind the reader that the prior-processing of major-element data by SINCLAS is a required step towards the correct use of our diagrams. The remaining part of 
Table 9. Sample locations for the application examples.

\begin{tabular}{|c|c|c|c|c|c|c|}
\hline $\begin{array}{l}\text { Site } \\
\#\end{array}$ & $\begin{array}{l}\text { Figure } \\
\quad \#\end{array}$ & $\begin{array}{l}\text { Longitude } \\
\left({ }^{\circ}\right)\end{array}$ & $\begin{array}{l}\text { Latitude } \\
\quad\left({ }^{\circ}\right)\end{array}$ & Country/Province & $\begin{array}{l}\text { No. of } \\
\text { samples }\end{array}$ & Reference \\
\hline \multicolumn{7}{|c|}{ Southern Mexico data used for constructing the Excel template } \\
\hline 357 & 3 & 259.52 & 20.45 & Mexican Volcanic Belt (central part) & 3 & Verma $(2002)$ \\
\hline 358 & 3 & 260.84 & 20.49 & $\begin{array}{l}\text { Mexican Volcanic Belt (central part, } \\
\text { Hidalgo state) }\end{array}$ & 4 & Verma (2002) \\
\hline 359 & 3 & 261.935 & 19.473 & $\begin{array}{l}\text { Mexican Volcanic Belt (Volcán La } \\
\text { Malinche area) }\end{array}$ & $1^{*}$ & Verma (2002) \\
\hline 360 & 3 & 262.538 & 19.272 & $\begin{array}{l}\text { Mexican Volcanic Belt (Las } \\
\text { Derrumbadas area) }\end{array}$ & $3^{*}$ & Verma (2002) \\
\hline 361 & 3 & 264.818 & 18.522 & Los Tuxtlas volcanic field & $2^{* *}$ & Verma (2002) \\
\hline 362 & 3 & 266.767 & 17.350 & El Chichón volcano & 1 & Verma (2002) \\
\hline \multicolumn{7}{|c|}{ Eastern part of the Mexican Volcanic Belt } \\
\hline 363 & 3 & 261.869 & 19.804 & $\begin{array}{l}\text { Mexican Volcanic Belt (Acoculco } \\
\text { caldera) }\end{array}$ & 2 & Verma (2001) \\
\hline 359 & 3 & 261.935 & 19.473 & $\begin{array}{l}\text { Mexican Volcanic Belt (Volcán La } \\
\text { Malinche area) }\end{array}$ & $1^{*}$ & Verma (2002) \\
\hline 364 & 3 & 262.479 & 19.708 & $\begin{array}{l}\text { Mexican Volcanic Belt (Los Humeros } \\
\text { caldera) }\end{array}$ & 5 & Ferriz and Mahood (1987) \\
\hline 365 & 3 & 262.535 & 19.646 & $\begin{array}{l}\text { Mexican Volcanic Belt (Los Humeros } \\
\text { caldera) }\end{array}$ & 4 & Verma (2000b) \\
\hline 360 & 3 & 262.538 & 19.272 & $\begin{array}{l}\text { Mexican Volcanic Belt (Las } \\
\text { Derrumbadas area) }\end{array}$ & $3^{*}$ & Verma (2002) \\
\hline 366 & 3 & 262.7 & 19.5 & Mexican Volcanic Belt (eastern part) & 8 & Demant (1981) \\
\hline 367 & 3 & 263.03 & 19.61 & Mexican Volcanic Belt (eastern part) & 17 & $\begin{array}{l}\text { Siebert and Carrasco- } \\
\text { Núñez }(2002)\end{array}$ \\
\hline 368 & 3 & 263.21 & 19.68 & Mexican Volcanic Belt (eastern part) & 12 & Gómez-Tuena et al (2003) \\
\hline 369 & 3 & 263.28 & 19.61 & $\begin{array}{l}\text { Mexican Volcanic Belt (eastern part, } \\
\text { including the easternmost Palma Sola } \\
\text { area) }\end{array}$ & 62 & Negendank et al (2002) \\
\hline \multicolumn{7}{|c|}{ Los Tuxtlas Volcanic Field (Mexico) } \\
\hline 370 & 3 & 264.981 & 18.431 & Los Tuxtlas volcanic field & 3 & Verma et al (1993) \\
\hline 371 & 3 & 264.9 & 18.5 & Los Tuxtlas volcanic field & 23 & Nelson et al (1995) \\
\hline 372 & 3 & 264.884 & 18.495 & Los Tuxtlas volcanic field & 14 & Verma (2006) \\
\hline 361 & 3 & 264.818 & 18.522 & Los Tuxtlas volcanic field & $2^{* *}$ & Verma (2002) \\
\hline \multicolumn{7}{|c|}{ Eastern Alkaline Province (Mexico) } \\
\hline 373 & 3 & 260.4 & 25.0 & Sierra de Picachos (Mexico) & 8 & Morton-Bermea (1990) \\
\hline 374 & 3 & 261.3 & 24.2 & Sierra San Carlos (Mexico) & 19 & Nick (1988) \\
\hline 375 & 3 & 261.3 & 24.2 & Sierra San Carlos (Mexico) & 4 & $\begin{array}{l}\text { Treviño-Cázares et al } \\
\text { (2005) }\end{array}$ \\
\hline 376 & 3 & 261.4 & 23.0 & Sierra de Tamaulipas (Mexico) & 5 & Orozco-Esquivel (1995) \\
\hline 377 & 3 & 261.4 & 23.0 & Sierra de Tamaulipas (Mexico) & 41 & Ramırez-Fernández (1996) \\
\hline 378 & 3 & 261.4 & 23.0 & Sierra de Tamaulipas (Mexico) & 2 & Robin and Tournon (1978) \\
\hline 379 & 3 & 262.0 & 20.0 & Pachuca (Mexico) & 7 & Orozco-Esquivel (1995) \\
\hline 380 & 3 & 262.0 & 22.0 & Tampico Plain (Mexico) & 4 & Robin and Tournon (1978) \\
\hline 381 & 3 & 262.5 & 20.5 & Poza Rica (Mexico) & 5 & Orozco-Esquivel (1995) \\
\hline \multicolumn{7}{|c|}{ Continental Arc (Andes) } \\
\hline 382 & 4 & 288.3 & -37.3 & Antuco (Chile) & 1 & Deruelle (1982) \\
\hline 383 & 4 & 287.2 & -40.9 & Osorno (Chile) & 1 & Deruelle (1982) \\
\hline 384 & 4 & 287.1 & -41.3 & Calbuco (Chile) & 1 & Deruelle (1982) \\
\hline 385 & 4 & 289.7 & -44.9 & El Pedrero (Argentina) & 1 & Deruelle (1982) \\
\hline 386 & 4 & 289.5 & -36.1 & Laguna de Maule volcano (Chile) & 1 & Frey et al (1984) \\
\hline 387 & 4 & 287.8 & -40.5 & Puyehue-Cordon Caulle (Chile) & 7 & Gerlach et al (1988) \\
\hline
\end{tabular}


Table 9. (Continued).

\begin{tabular}{|c|c|c|c|c|c|c|}
\hline $\begin{array}{c}\text { Site } \\
\#\end{array}$ & $\underset{\#}{\text { Figure }}$ & $\begin{array}{c}\text { Longitude } \\
\left(^{\circ}\right)\end{array}$ & $\begin{array}{l}\text { Latitude } \\
\left(\left(^{\circ}\right)\right.\end{array}$ & Country/Province & $\begin{array}{c}\text { No. of } \\
\text { samples }\end{array}$ & Reference \\
\hline 388 & 4 & 288.0 & -39.3 & Pichares (Chile) & 1 & Hickey-Vargas et al (1989) \\
\hline 389 & 4 & 288.0 & -39.3 & Caburga (Chile) & 2 & Hickey-Vargas et al (1989) \\
\hline 390 & 4 & 288.0 & -39.3 & Cerro Redondo (Chile) & 1 & Hickey-Vargas et al (1989) \\
\hline 391 & 4 & 288.0 & -39.3 & Lanin volcano (Chile) & 2 & Hickey-Vargas et al (1989) \\
\hline 392 & 4 & 287.7 & -40.6 & Puyehue (Chile) & 6 & Hickey et al (1986) \\
\hline 393 & 4 & 288.3 & -38.7 & Llaima (Chile) & 1 & Hickey et al (1986) \\
\hline 394 & 4 & 288.3 & -39.5 & Quetrupillan (Chile) & 1 & Hickey et al (1986) \\
\hline 395 & 4 & 287.7 & -40.7 & Antillanca (Chile) & 3 & Hickey et al (1986) \\
\hline 396 & 4 & 289.0 & -41.8 & Yate (Chile) & 1 & López-Escobar et al (1993) \\
\hline 397 & 4 & 289.0 & -41.9 & Hualaihue (Chile) & 1 & López-Escobar et al (1993) \\
\hline 398 & 4 & 289.0 & -43.2 & Corcovado (Chile) & 1 & López-Escobar et al (1993) \\
\hline 399 & 4 & 289.0 & -45.1 & Cay (Chile) & 5 & López-Escobar et al (1993) \\
\hline 400 & 4 & 289.0 & -45.1 & Maca (Chile) & 1 & López-Escobar et al (1993) \\
\hline 401 & 4 & 289.0 & -46.0 & Hudson (Chile) & 1 & López-Escobar et al (1993) \\
\hline 402 & 4 & 287.7 & -40.6 & Puyehue (Chile) & 2 & Tormey et al (1991) \\
\hline
\end{tabular}

Continental Arc (Guatemala, El Salvador, Nicaragua, Honduras, and NW Costa Rica)

\begin{tabular}{|c|c|c|c|c|c|c|}
\hline 403 & 3 & 269.4 & 14.38 & Pacaya (Guatemala) & 2 & Carr's website ${ }^{* * *}$ \\
\hline 404 & 3 & 269.5 & 14.3 & Pacaya (Guatemala) & 1 & Carr (1984) \\
\hline 405 & 3 & 269.5 & 14.3 & Pacaya (Guatemala) & 3 & $\begin{array}{l}\text { Bardintzeff and Deniel } \\
(1992)\end{array}$ \\
\hline 406 & 3 & 269.5 & 14.3 & Cuilapa (Guatemala) & 1 & Carr et al (1990) \\
\hline 407 & 3 & 269.5 & 14.3 & Pagaya (Guatemala) & 1 & Carr et al (1990) \\
\hline 408 & 3 & 269.50 & 14.3 & Cuilapa (Guatemala) & 3 & Carr's website ${ }^{* * *}$ \\
\hline 409 & 3 & 270.20 & 13.84 & Apaneca (El Salvador) & 2 & Carr's website ${ }^{* * *}$ \\
\hline 410 & 3 & 270.37 & 13.82 & Izalco (El Salvador) & 1 & Carr's website ${ }^{* * *}$ \\
\hline 411 & 3 & 270.37 & 13.83 & Cerro Verde (El Salvador) & 1 & Carr's website*** \\
\hline 412 & 3 & 271.23 & 13.72 & Apastape (El Salvador) & 1 & Carr's website ${ }^{* * *}$ \\
\hline 413 & 3 & 271.50 & 13.5 & Tecapa (El Salvador) & 1 & Carr's website ${ }^{* * *}$ \\
\hline 414 & 3 & 271.53 & 13.42 & Usulután (El Salvador) & 1 & Carr's website ${ }^{* * *}$ \\
\hline 415 & 3 & 271.73 & 13.43 & San Miguel (El Salvador) & 3 & Carr's website ${ }^{* * *}$ \\
\hline 416 & 3 & 272.15 & 13.28 & Conchagua (El Salvador) & 3 & Carr's website ${ }^{* * *}$ \\
\hline 417 & 3 & 273.0 & 12.6 & San Cristóbal (Nicaragua) & 9 & Hazlett (1987) \\
\hline 418 & 3 & 273.0 & 12.7 & San Cristóbal (Nicaragua) & 13 & Carr's website ${ }^{* * *}$ \\
\hline 419 & 3 & 273.15 & 12.6 & Telica (Nicaragua) & 16 & Carr's website ${ }^{* * *}$ \\
\hline 420 & 3 & 273.25 & 12.55 & Rota (Nicaragua) & 2 & Carr's website ${ }^{* * *}$ \\
\hline 421 & 3 & 273.30 & 12.50 & Cerro Negro (Nicaragua) & 21 & Carr's website ${ }^{* * *}$ \\
\hline 422 & 3 & 273.32 & 12.50 & Las Pilas (Nicaragua) & 2 & Carr's website ${ }^{* * *}$ \\
\hline 423 & 3 & 273.47 & 12.42 & Momotombo (Nicaragua) & 1 & Carr's website ${ }^{* * *}$ \\
\hline 424 & 3 & 273.68 & 12.11 & Nejapa (Nicaragua) & 15 & Carr's website ${ }^{* * *}$ \\
\hline 425 & 3 & 273.8 & 12.0 & Nejapa-Granada (Nicaragua) & 13 & Walker et al (1990) \\
\hline 426 & 3 & 273.8 & 12.0 & Granada (Nicaragua) & 2 & Walker et al (2001) \\
\hline 427 & 3 & 273.8 & 12.0 & Apoyo (Nicaragua) & 1 & Walker et al (2001) \\
\hline 428 & 3 & 273.8 & 12.0 & Nejapa (Nicaragua) & 5 & Walker et al (2001) \\
\hline 429 & 3 & 273.3 & 12.4 & Cerro Negro (Nicaragua) & 1 & Walker et al (2001) \\
\hline 430 & 3 & 273.85 & 11.98 & Masaya (Nicaragua) & 20 & Carr's website ${ }^{* * *}$ \\
\hline 431 & 3 & 273.8 & 12.0 & Masaya (Nicaragua) & 1 & Carr (1984) \\
\hline 432 & 3 & 273.95 & 11.93 & Apoyo (Nicaragua) & 2 & Carr's website ${ }^{* * *}$ \\
\hline 433 & 3 & 274.00 & 11.88 & Granada (Nicaragua) & 4 & Carr's website ${ }^{* * *}$ \\
\hline 434 & 3 & 274.02 & 11.83 & Mombacho (Nicaragua) & 1 & Carr's website ${ }^{* * *}$ \\
\hline
\end{tabular}


Table 9. (Continued).

\begin{tabular}{|c|c|c|c|c|c|c|}
\hline $\begin{array}{l}\text { Site } \\
\#\end{array}$ & $\underset{\#}{\text { Figure }}$ & $\begin{array}{l}\text { Longitude } \\
\qquad\left(^{\circ}\right)\end{array}$ & $\begin{array}{l}\text { Latitude } \\
\quad\left(^{\circ}\right)\end{array}$ & Country/Province & $\begin{array}{l}\text { No. of } \\
\text { samples }\end{array}$ & Reference \\
\hline 435 & 3 & 274.53 & 10.98 & Orosi (Costa Rica) & 1 & Carr's website ${ }^{* * *}$ \\
\hline 436 & 3 & 274.67 & 10.83 & Rincón (Costa Rica) & 2 & Carr's website*** \\
\hline 437 & 3 & 272.02 & 14.98 & Yohoa (Honduras) & 10 & Carr's website ${ }^{* * *}$ \\
\hline 438 & 3 & 272.40 & 13.30 & Zacate Grande (Honduras) & 2 & Carr's website ${ }^{* * *}$ \\
\hline \multicolumn{7}{|c|}{ Deccan Traps (India) } \\
\hline 439 & 1 & 70.4 & 22.0 & Kathiawar (India) & 12 & Melluso et al (1995) \\
\hline 440 & 1 & 73.0 & 19.0 & Deccan Traps (India) & 3 & Peng et al (1994) \\
\hline 441 & 1 & 73.0 & 21.7 & Rajpipla (India) & 6 & Melluso et al (1995) \\
\hline 442 & 1 & 73.1 & 19.3 & Deccan Traps (India) & 1 & Melluso et al (2004) \\
\hline 443 & 1 & 73.6 & 19.6 & Deccan Traps (India) & 1 & Melluso et al (2004) \\
\hline 444 & 1 & 73.6 & 20.7 & Deccan Traps (India) & 1 & Melluso et al (2004) \\
\hline 445 & 1 & 73.7 & 20.0 & Deccan western (India) & 5 & Duraiswami et al (2004) \\
\hline 446 & 1 & 73.7 & 20.7 & Deccan Traps (India) & 1 & Melluso et al (2004) \\
\hline 447 & 1 & 73.7 & 22.7 & Pavagadh (India) & 4 & Melluso et al (1995) \\
\hline 448 & 1 & 73.7 & 20.6 & Deccan Traps (India) & 1 & Melluso et al (2004) \\
\hline 449 & 1 & 73.7 & 19.8 & Deccan Traps (India) & 1 & Melluso et al (2004) \\
\hline 450 & 1 & 73.9 & 20.5 & Deccan Traps (India) & 1 & Melluso et al (2004) \\
\hline 451 & 1 & 73.9 & 20.1 & Deccan Traps (India) & 1 & Melluso et al (2004) \\
\hline 452 & 1 & 73.9 & 20.3 & Deccan Traps (India) & 1 & Melluso et al (2004) \\
\hline 453 & 1 & 74.0 & 20.3 & Deccan Traps (India) & 1 & Melluso et al (2004) \\
\hline 454 & 1 & 74.1 & 19.6 & Deccan western (India) & 26 & Bondre et al (2006) \\
\hline 455 & 1 & 74.7 & 20.5 & Deccan Traps (India) & 1 & Melluso et al (2004) \\
\hline 456 & 1 & 74.9 & 19.5 & Deccan Traps (India) & 1 & Melluso et al (2004) \\
\hline 457 & 1 & 74.9 & 20.1 & Deccan Traps (India) & 4 & Melluso et al (2004) \\
\hline 458 & 1 & 74.9 & 19.4 & Deccan Traps (India) & 1 & Melluso et al (2004) \\
\hline 459 & 1 & 75.0 & 20.5 & Deccan Traps (India) & 4 & Melluso et al (2004) \\
\hline 460 & 1 & 75.0 & 19.3 & Deccan Traps (India) & 1 & Melluso et al (2004) \\
\hline 461 & 1 & 75.1 & 19.9 & Deccan Traps (India) & 4 & Melluso et al (2004) \\
\hline 462 & 1 & 75.1 & 21.6 & Bijasan Ghat (India) & 33 & Sheth et al (2004) \\
\hline 463 & 1 & 75.2 & 19.7 & Deccan Traps (India) & 2 & Melluso et al (2004) \\
\hline 464 & 1 & 75.3 & 19.8 & Deccan Traps (India) & 1 & Melluso (2004) \\
\hline 465 & 1 & 75.4 & 20.9 & Deccan Traps (India) & 1 & Melluso et al (2004) \\
\hline 466 & 1 & 75.5 & 19.7 & Deccan Traps (India) & 2 & Melluso et al (2004) \\
\hline 467 & 1 & 75.6 & 20.1 & Deccan Traps (India) & 1 & Melluso et al (2004) \\
\hline 468 & 1 & 75.8 & 19.5 & Deccan Traps (India) & 2 & Melluso et al (2004) \\
\hline \multicolumn{7}{|c|}{ Malani igneous suite (India) } \\
\hline 469 & 1 & 72.4 & 17.5 & Malani, Diri-Gurpratat (India) & 1 & $\begin{array}{l}\text { Maheshwari et al (1996), } \\
\text { cited in table } 6 \mathrm{~B} \text { of Sharma } \\
(2004)\end{array}$ \\
\hline 470 & 1 & 72.4 & 17.5 & Malani suite, Kundal (India) & 8 & $\begin{array}{l}\text { Singh and Vallinayagam } \\
(2004)\end{array}$ \\
\hline 471 & 1 & 72.18 & 24.82 & Malani, Golwara (India) & 2 & $\begin{array}{l}\text { Bhushan and } \\
\text { Chandrasekaran (2002) }\end{array}$ \\
\hline 472 & 1 & 72.71 & 25.20 & Malani, Bagra (India) & 1 & $\begin{array}{l}\text { Bhushan and } \\
\text { Chandrasekaran (2002) }\end{array}$ \\
\hline 473 & 1 & 71.87 & 26.23 & Malani, Ramdevjee (India) & 1 & $\begin{array}{l}\text { Bhushan and } \\
\text { Chandrasekaran (2002) }\end{array}$ \\
\hline 474 & 1 & 72.13 & 25.78 & Malani, Simaliya (India) & 3 & $\begin{array}{l}\text { Bhushan and } \\
\text { Chandrasekaran (2002) }\end{array}$ \\
\hline
\end{tabular}


Table 9. (Continued).

\begin{tabular}{ccccccc}
\hline $\begin{array}{c}\text { Site } \\
\#\end{array}$ & $\begin{array}{c}\text { Figure } \\
\#\end{array}$ & $\begin{array}{c}\text { Longitude } \\
\left({ }^{\circ}\right)\end{array}$ & $\begin{array}{c}\text { Latitude } \\
\left({ }^{\circ}\right)\end{array}$ & \multicolumn{1}{c}{ Country/Province } & $\begin{array}{c}\text { No. of } \\
\text { samples }\end{array}$ & Reference \\
\hline 475 & 1 & 72.08 & 24.88 & Malani, Karara (India) & 1 & $\begin{array}{l}\text { Bhushan and } \\
\text { Chandrasekaran (2002) }\end{array}$ \\
476 & 1 & 71.23 & 25.72 & Malani, Jasai (India) & 1 & $\begin{array}{l}\text { Bhushan and } \\
\text { Chandrasekaran (2002) } \\
\text { Bhushan and } \\
\text { Chandrasekaran (2002) }\end{array}$ \\
\hline
\end{tabular}

\begin{tabular}{|c|c|c|c|c|c|c|}
\hline \multicolumn{7}{|c|}{ Bastar craton dykes (India) } \\
\hline 478 & 1 & 81.5 & 19.0 & Bastar craton (India) Bastanar & 6 & Srivastava et al (1996) \\
\hline 479 & 1 & 81.3 & 18.9 & Bastar craton (India) Dantewara & 2 & Srivastava et al (1996) \\
\hline 480 & 1 & 81.6 & 18.8 & Bastar craton (India) Katekalyan & 1 & Srivastava et al (1996) \\
\hline 481 & 1 & 81.72 & 18.85 & Southern Bastar craton (India) & 2 & Srivastava and Singh (2004) \\
\hline 482 & 1 & 81.72 & 18.98 & Southern Bastar craton (India) & 1 & Srivastava and Singh (2004) \\
\hline 483 & 1 & 81.71 & 18.85 & Southern Bastar craton (India) & 2 & Srivastava and Singh (2004) \\
\hline 484 & 1 & 81.70 & 18.98 & Southern Bastar craton (India) & 1 & Srivastava and Singh (2004) \\
\hline 485 & 1 & 81.69 & 18.87 & Southern Bastar craton (India) & 1 & Srivastava and Singh (2004) \\
\hline 486 & 1 & 81.69 & 18.98 & Southern Bastar craton (India) & 1 & Srivastava and Singh (2004) \\
\hline 487 & 1 & 81.69 & 18.86 & Southern Bastar craton (India) & 1 & Srivastava and Singh (2004) \\
\hline 488 & 1 & 81.68 & 18.98 & Southern Bastar craton (India) & 1 & Srivastava and Singh (2004) \\
\hline 489 & 1 & 81.67 & 18.99 & Southern Bastar craton (India) & 1 & Srivastava and Singh (2004) \\
\hline 490 & 1 & 81.63 & 18.98 & Southern Bastar craton (India) & 1 & Srivastava and Singh (2004) \\
\hline 491 & 1 & 81.63 & 18.85 & Southern Bastar craton (India) & 1 & Srivastava and Singh (2004) \\
\hline 492 & 1 & 81.60 & 18.95 & Southern Bastar craton (India) & 1 & Srivastava and Singh (2004) \\
\hline 493 & 1 & 81.59 & 18.95 & Southern Bastar craton (India) & 1 & Srivastava and Singh (2004) \\
\hline 494 & 1 & 81.57 & 18.96 & Southern Bastar craton (India) & 1 & Srivastava and Singh (2004) \\
\hline 495 & 1 & 81.56 & 18.97 & Southern Bastar craton (India) & 2 & Srivastava and Singh (2004) \\
\hline 496 & 1 & 81.55 & 18.98 & Southern Bastar craton (India) & 1 & Srivastava and Singh (2004) \\
\hline 497 & 1 & 81.54 & 18.97 & Southern Bastar craton (India) & 2 & Srivastava and Singh (2004) \\
\hline 498 & 1 & 81.53 & 18.98 & Southern Bastar craton (India) & 1 & Srivastava and Singh (2004) \\
\hline 499 & 1 & 81.53 & 18.97 & Southern Bastar craton (India) & 3 & Srivastava and Singh (2004) \\
\hline 500 & 1 & 81.52 & 18.98 & Southern Bastar craton (India) & 3 & Srivastava and Singh (2004) \\
\hline 501 & 1 & 81.52 & 18.99 & Southern Bastar craton (India) & 1 & Srivastava and Singh (2004) \\
\hline 502 & 1 & 81.52 & 18.97 & Southern Bastar craton (India) & 3 & Srivastava and Singh (2004) \\
\hline 503 & 1 & 81.52 & 18.91 & Southern Bastar craton (India) & 1 & Srivastava and Singh (2004) \\
\hline 504 & 1 & 81.51 & 18.98 & Southern Bastar craton (India) & 2 & Srivastava and Singh (2004) \\
\hline 505 & 1 & 81.51 & 18.99 & Southern Bastar craton (India) & 1 & Srivastava and Singh (2004) \\
\hline 506 & 1 & 81.51 & 18.91 & Southern Bastar craton (India) & 1 & Srivastava and Singh (2004) \\
\hline 507 & 1 & 81.50 & 18.98 & Southern Bastar craton (India) & 2 & Srivastava and Singh (2004) \\
\hline 508 & 1 & 81.49 & 18.98 & Southern Bastar craton (India) & 2 & Srivastava and Singh (2004) \\
\hline 509 & 1 & 81.48 & 18.97 & Southern Bastar craton (India) & 1 & Srivastava and Singh (2004) \\
\hline 510 & 1 & 81.47 & 18.98 & Southern Bastar craton (India) & 1 & Srivastava and Singh (2004) \\
\hline 511 & 1 & 81.46 & 18.98 & Southern Bastar craton (India) & 1 & Srivastava and Singh (2004) \\
\hline 512 & 1 & 81.46 & 18.90 & Southern Bastar craton (India) & 1 & Srivastava and Singh (2004) \\
\hline 513 & 1 & 81.46 & 18.91 & Southern Bastar craton (India) & 1 & Srivastava and Singh (2004) \\
\hline 514 & 1 & 81.43 & 18.92 & Southern Bastar craton (India) & 1 & Srivastava and Singh (2004) \\
\hline 515 & 1 & 81.40 & 18.94 & Southern Bastar craton (India) & 1 & Srivastava and Singh (2004) \\
\hline 516 & 1 & 81.40 & 18.93 & Southern Bastar craton (India) & 1 & Srivastava and Singh (2004) \\
\hline 517 & 1 & 81.39 & 18.93 & Southern Bastar craton (India) & 1 & Srivastava and Singh (2004) \\
\hline 518 & 1 & 81.38 & 18.94 & Southern Bastar craton (India) & 2 & Srivastava and Singh (2004) \\
\hline
\end{tabular}

${ }^{*}$ Also used in evaluating the eastern part of the MVB (E-MVB); ${ }^{* *}$ also used in evaluating the Los Tuxtlas volcanic field; *** M J Carr's website (http://www.rci.rutgers.edu/ carr; June 2004). 

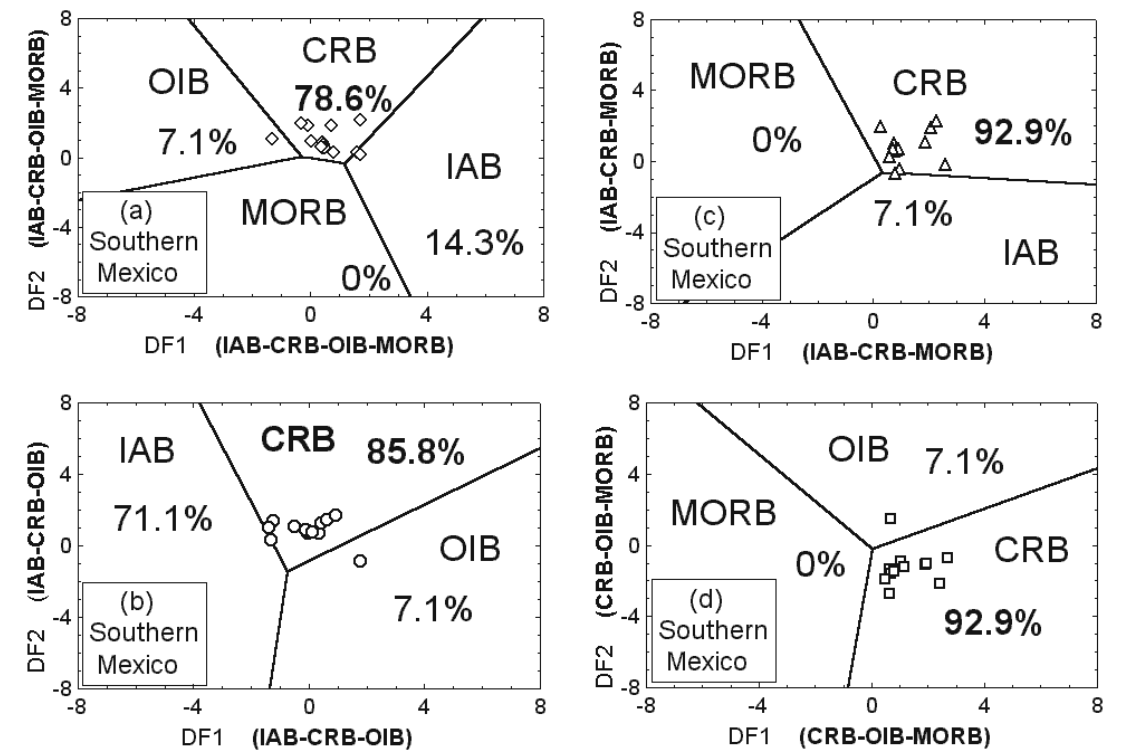

Figure 7. Four (of the five) pertinent discriminant function diagrams for southern Mexico partial data (for 14 samples of basic and ultrabasic rocks tabulated as 'new data' by Verma 2002). Note most samples fall in the CRB field; the corresponding percentages in each diagram and for each tectonic setting are the \% success of the classification for the samples under evaluation (e.g., CRB $78.6 \%$ for the first diagram). See the Excel template example and the text for more details on the use of these diagrams; also see the Excel template for more details on the data processing. (a) Four-groups IAB, CRB, OIB, and MORB (1-2-3-4); (b) Three-groups IAB, CRB, and OIB (1-2-3); (c) Three-groups IAB, CRB, and MORB (1-2-4); and (d) Three-groups CRB, OIB, and MORB (2-3-4).

the Excel sheet is to calculate ratios of majorelement concentrations to $\mathrm{SiO}_{2}$ concentration, and then to carry out the $\log _{\mathrm{e}}$-transformation of the ratio data. Thus, in this first sheet, the basic calculations are performed, whereas the remaining sheets present the respective diagrams. Finally, the two discriminant functions DF1 and DF2 for a given diagram (for four-groups named as figure E5 and for three-groups at a time named as figure E6a, E6b, E6c, and E6d) are calculated using the appropriate factors listed in tables 6 and 7 . The corresponding DF1 and DF2 data are then manually transferred ('copy and paste') to the other Excel sheets and the new diagrams prepared (by 'dragging' the data into the respective figure) and adopted for specific applications and interpretations.

The four diagrams applicable for the southern Mexico data are presented in figure $7(\mathrm{a}-\mathrm{d})$. The selected diagrams should include the inferred setting, while the diagram which does not have this indicated setting is not considered any further. In the case of southern Mexico, the indicated setting is CRB (see most samples fall in the CRB field in all four diagrams in figure $7 \mathrm{a}-\mathrm{d}$ ). Therefore, the remaining diagram (in this case, the figure with IAB-OIB-MORB settings; see figure 6c) from the set of five proposed diagrams (figures 5 and $6 \mathrm{a}^{-}$ d) is eliminated from the discussion. The \% success rates for the CRB field in all diagrams are very high varying from 78.6 to $92.9 \%$; these success rates are similar to those obtained from the training and testing sets (see table 5). This strongly suggests that the inferred tectonic setting for the basic volcanism in southern Mexico is a continental rift (CRB), and not an arc (IAB) or oceanisland (OIB). Note also that we have used a relatively small number of samples to illustrate the use of our diagrams. We present three more case histories of complex areas of Mexico (the Mexican Volcanic Belt, the Los Tuxtlas volcanic field, and the Eastern Alkaline Province) to confirm these results, using a much larger and more representative database.

\subsection{Mexican Volcanic Belt (MVB) as a province of a complex or controversial tectonic setting}

This Miocene to Recent volcanic province in Mexico has had a highly controversial nature for its tectonic setting (see for example, Sheth et al 2000, 2002; Verma 2002, 2004; and references therein). The suggested tectonic setting for the MVB has varied from the conventional arc (continental arc) to OIB and CRB settings, in spite of the ongoing subduction. The Excel template example presented above clearly points to a CRB setting not only for the MVB but also for the whole of southern Mexico; this interpretation is fully consistent with Verma (2002) who used several other lines of evidence and an extensive database from Mexico 

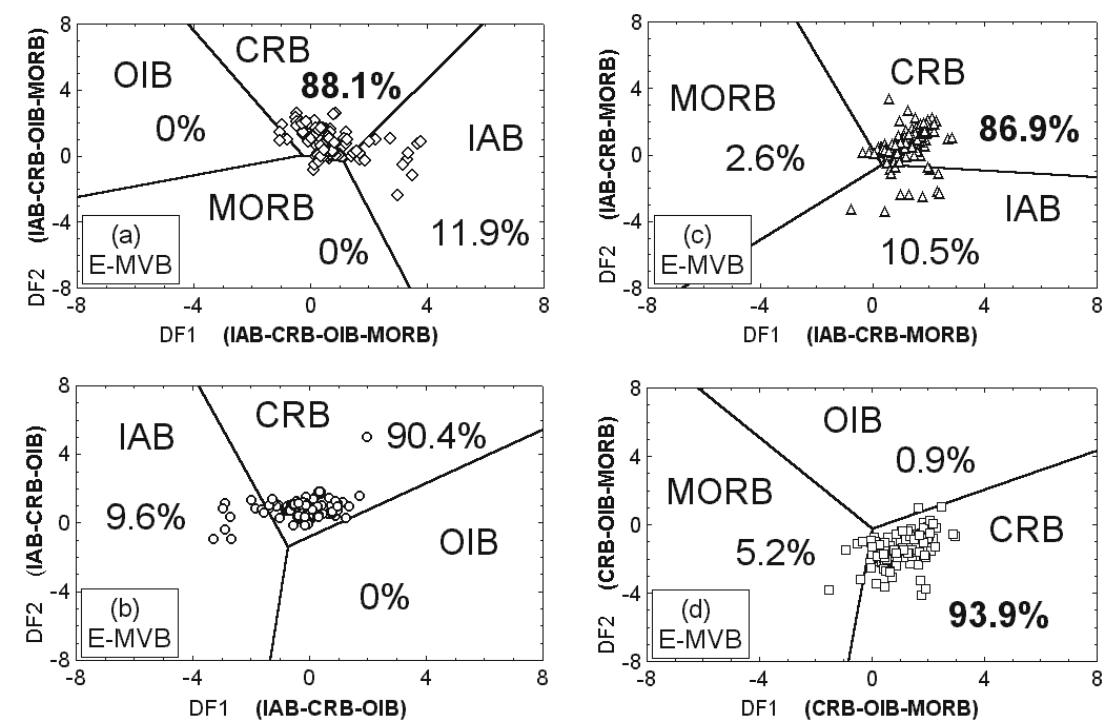

Figure 8. Four pertinent diagrams for 114 samples of basic and ultrabasic rocks from the eastern part of the Mexican Volcanic Belt. Note most samples fall in the CRB field (the corresponding \% success rates for this tectonic setting - 86.9 to $93.9 \%$ - are extremely high). The figure parts (a) to (d) are the same as in figure 7 .

and Central America, to conclude a rift setting for all of southern Mexico.

To further illustrate the usefulness of our new diagrams we plot the data compiled by Verma (2004) for the eastern part of the MVB (E-MVB) in our new diagrams (see table 9 for sample locations). We restrict our present application to this part of the MVB mainly for the following reasons:

- this is the region where the subductionrelationship (arc setting) of the MVB was seriously questioned for the first time more than 20 years ago (Verma 1983);

- this region shows an overlap with the Eastern Alkaline Province (EAP) of Mexico (e.g., Verma 2006);

- a 'back-arc setting' has been implicitly proposed as an explanation for the unusual chemistry of lavas from this area (see 'behind-the-arc' in the title of the paper by Siebert and Carrasco-Núñez 2002) - a nomenclature already questioned by Verma (2004, 2006), because the assignment of such a tectonic setting requires the presence of an 'arc' somewhere between this area and the respective trench, which unfortunately does not exist in southern Mexico; and

- a restricted area will help us to limit the number of samples to be evaluated for a better visual examination of the discrimination diagrams (114 samples used; table 9; figure 8a-d).

The basic and ultrabasic rock data from the E-MVB plotted in our discriminant function diagrams undoubtedly show that they represent a CRB setting; the \% success rates vary from 86.9 to
93.9\% - extremely high percentages for application in an 'unknown' or a complex setting.

\subsection{Other Mexican provinces}

\subsubsection{Los Tuxtlas volcanic field (LTVF)}

The tectonic setting of this Miocene to Recent volcanic province has also been controversial because different settings have been proposed for the LTVF: a typical arc setting (Thorpe 1977); an extensional setting (Robin 1976, 1982; Robin and Tournon 1978); and a 'hybrid' 'arc and back-arc' setting, both arc and back-arc being located at the same place (Nelson et al 1995). From a detailed geochemical study, recently Verma (2006) reached the conclusion that the LTVF represents an essentially extensional setting. In this context, it would be interesting to find out the indications of our new discriminant function diagrams.

We plotted the data for 42 samples of basic and ultrabasic rocks from the LTVF in figure $9(\mathrm{a}-\mathrm{d})$, which clearly shows that the LTVF represents a continental rift setting - the \% success varies from 83.3 to $97.6 \%$. Further, although the LTVF is an isolated area, these results and other lines of evidence also suggest that the LTVF belongs to the EAP (see more details in Verma 2006).

\subsubsection{Eastern Alkaline Province (EAP)}

This volcanic province (EAP) in north-east Mexico has been conventionally attributed to extensional tectonics along the Gulf of Mexico coast, i.e, there has been less controversy, until recently, regarding 

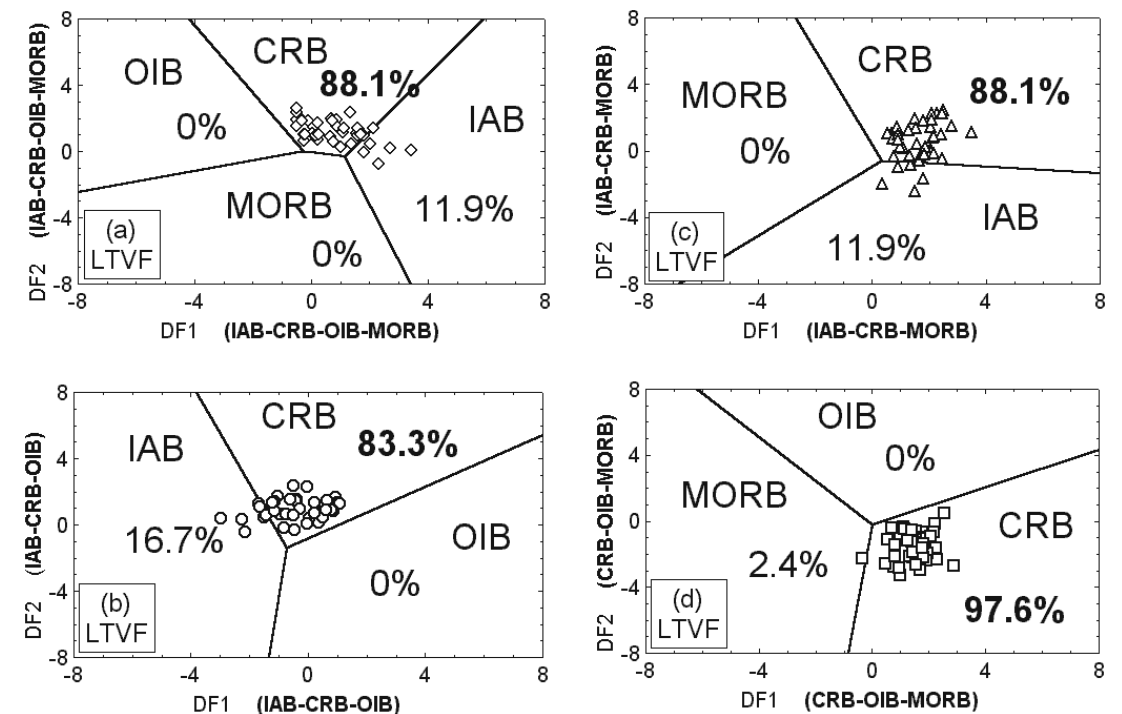

Figure 9. Four pertinent diagrams for 42 samples of basic and ultrabasic rocks from the Los Tuxtlas volcanic field (LTVF). Note most samples fall in the CRB field (as in figure 8, the corresponding \% success rates for this tectonic setting - 83.3 to $97.6 \%$ - are also extremely high). The figure parts (a) to (d) are the same as in figure 7 .
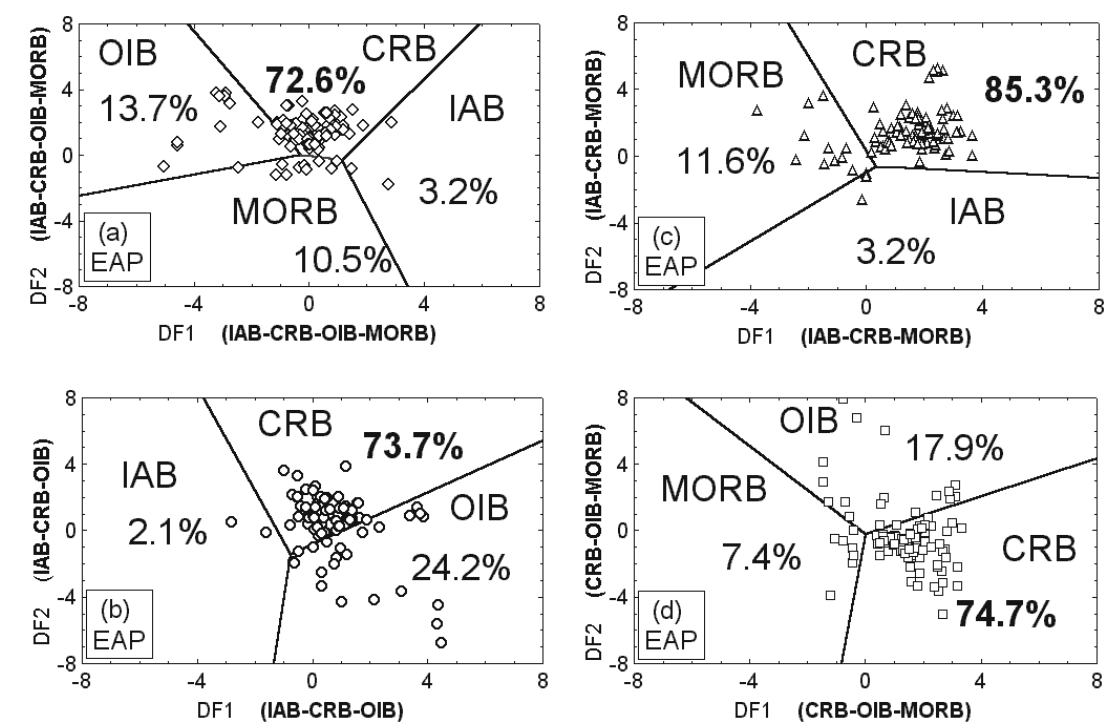

Figure 10. Four pertinent diagrams for 95 samples of basic and ultrabasic rocks from the Eastern Alkaline Province (EAP). Note most samples fall in the CRB field (the corresponding \% success rates for this tectonic setting - 72.6 to $85.3 \%-$ are high). The figure parts (a) to (d) are the same as in figure 7 .

its tectonic setting as the CRB setting than the other Mexican areas discussed in this paper (the MVB and the LTVF). The actual extensional faults were recognized by Robin (1982), as of Oligocene to Present and parallel to the Gulf of Mexico, but recently Ferrari et al (2005) attributed, instead, a major role to the older late Jurassic 'hidden' fault structures in this area. Ferrari et al (2005), in addition, suggested a relationship of the EAP with the current subduction along the Pacific coast and denied its rift-related origin.
We used a recent compilation by Verma (2006) to apply our new discriminant function diagrams for inferring the tectonic setting for this province, also now characterized with a controversial tectonic setting as the other two Mexican areas (see 5.2 and 5.3.1 above). We plotted the data for basic and ultrabasic rocks (95 samples) from the EAP in figure $10(a-d)$. This figure clearly shows that the EAP represents a continental rift setting the $\%$ success varies from 72.6 to $85.3 \%$. These results, certainly, do not support the contention of 

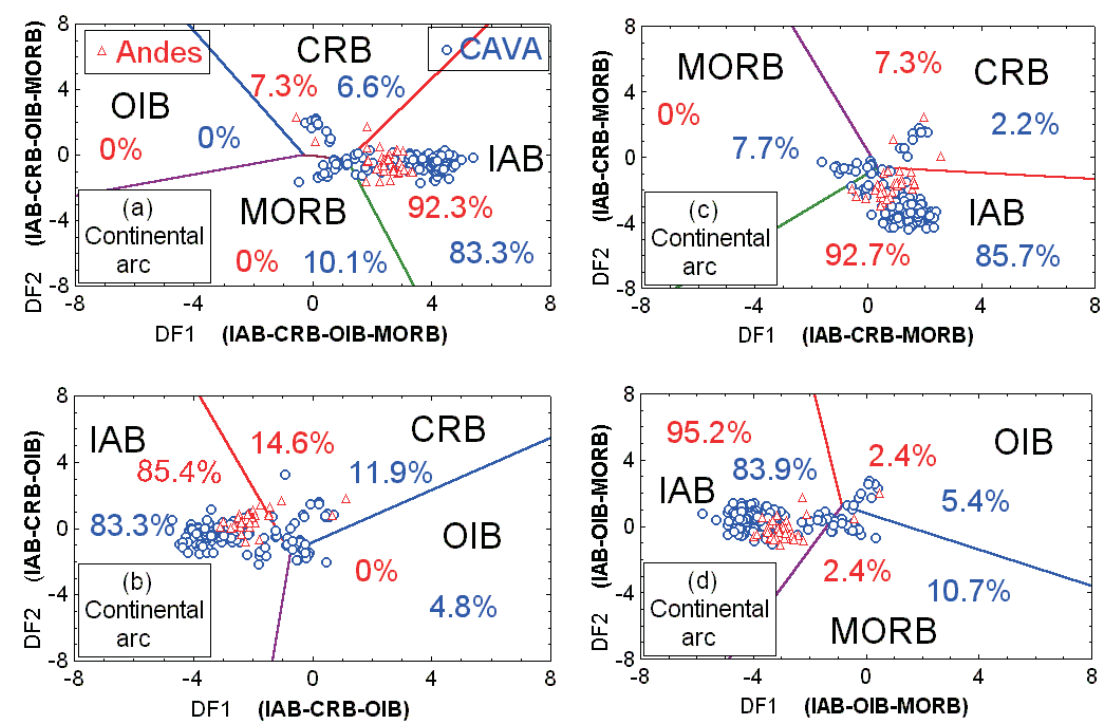

Figure 11. Four pertinent diagrams for 209 samples of basic rocks from two arcs (41 from the Los Andes continental arc - Andes, and 168 from the Central American Volcanic Arc - CAVA). Note most samples fall in the IAB field; the corresponding \% success rates for the Andes samples - 85.4 to $95.2 \%$ (see numbers in blue) - are extremely high, whereas for the CAVA they are high (83.3 to $85.9 \%$; see numbers in green). The figure parts (a) to (c) are the same as in figure 7 , but (d) is the three-groups IAB, OIB, and MORB (1-3-4).

Ferrari et al (2005) that the EAP does not have a rift-related origin, and instead support the recent work by Verma (2006) who also used numerous other tectonomagmatic discrimination diagrams as well as other evidence to show its rift-related origin.

\subsection{Continental arc setting}

We compiled basic rock samples (practically no ultrabasic rocks are reported from these areas) from two continental arcs (continental arc basic rocks - CAB setting) for testing if this missing $\mathrm{CAB}$ set (note our diagrams discriminate only the IAB, CRB, OIB, and MORB settings) preferably plots in the IAB setting. The Andean continental arc (Andes) is represented by 41 samples, whereas the Central American Volcanic Arc (CAVA) is depicted by 168 samples (figure 11a-d). Rocks from both arcs plot mainly ( 85.4 to $95.2 \%$ from the Andes and 83.3 to $85.9 \%$ from the CAVA) in the field of IAB, suggesting an IAB-type setting for these two provinces. Thus, basic rocks from the $\mathrm{CAB}$ and $\mathrm{IAB}$ settings have very similar majorelement chemistry. To distinguish them $(\mathrm{CAB}$ and $\mathrm{IAB}$ ), we probably would need to include trace elements in our discriminant analysis.

\subsection{Deccan flood basalt province - an example that should probably await further research}

This important volcanic province in India was represented in our application database by 124 samples of basic rocks from different locations (figure 1; table 9). These samples were plotted in our discriminant function diagrams (figure 12ad). The complex nature of the Deccan is evident from the four-group diagram (figure 12a), in which the samples plot in all four fields. However, the other three-group diagrams (figure $12 \mathrm{~b}-\mathrm{d}$ ) indicate the preference for the OIB setting in which about 68.6 to $79.8 \%$ samples plot. In any case, these \% success rates for this setting (OIB) are relatively low.

There may be several explanations for these results. First, Agrawal et al (2004) pointed out the difficulties of discriminant function diagrams when more fields such as all four are considered at a time, the consideration of less fields, for example, three at a time probably provides more reliable results. The second, probably a more plausible reason may be that the Deccan traps are considered as 'flood basalts', which may represent a distinctly different tectonic setting than the four settings we have considered in our diagrams. Because samples from this type of areas (flood basalts) were not explicitly included in our initial database, our diagrams should not be used for a setting expected from field work to be different from the four settings actually considered (the IAB, CRB, OIB, and MORB settings). In fact, samples from a different tectonic setting will fall close to or inside the field, for which they have more chemical (in this case, major-element) similarity, which in the case of the Deccan, seems to be with the OIB setting. It appears, therefore, that a priori knowledge, 

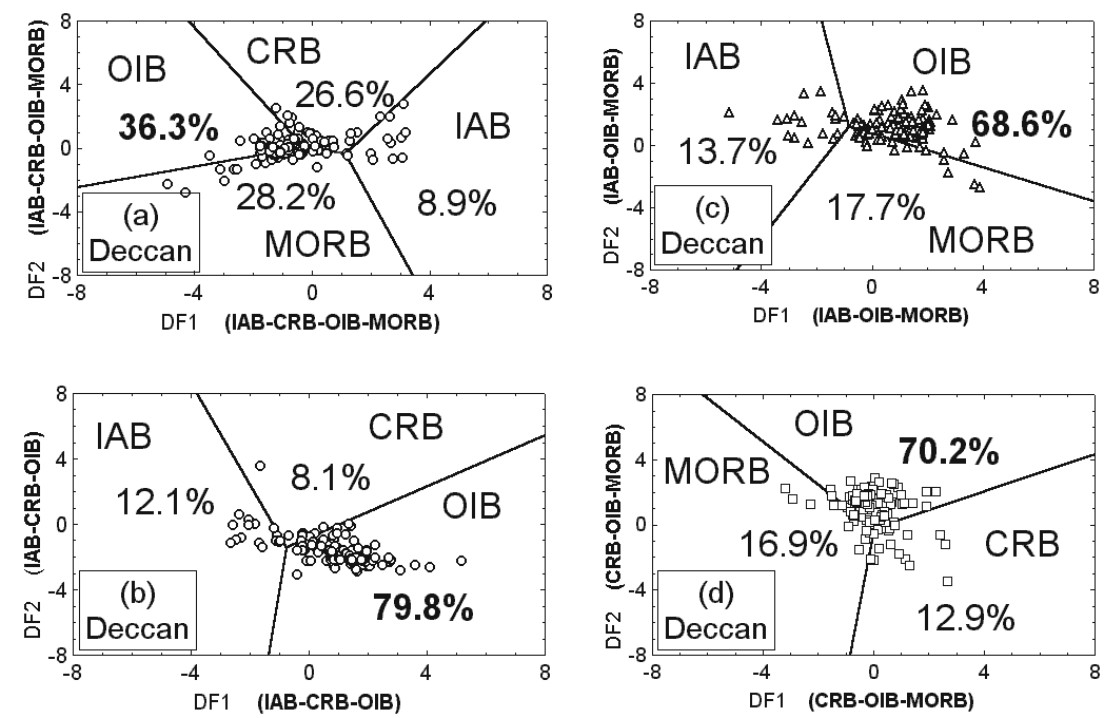

Figure 12. Four pertinent diagrams for 124 samples of basic and ultrabasic rocks from the Deccan flood basalt province, India. The figure parts (a), (b), and (d) are the same as in figure 7 , but (c) is the three-group IAB, OIB, and MORB $(1-3-4)$. See the text for the complexity of the interpretation.

probably from prior field work, that the samples actually belong to one of the tectonic settings of the diagram, is a necessary requirement in the use of these discriminant diagrams. Another reason for the lack of a more definite indication of the discriminant function diagrams for the Deccan case might be the highly complex and variable nature of the sources and processes operative in this vast province. To overcome this problem, in future we probably need to include more tectonic settings such as flood basalt or enriched-MORB in our database and then propose new discriminant function diagrams.

\subsection{Cratons from India}

Major-element data for basic rock samples from two cratonic areas (the Malani igneous complex from northwestern India and the Bastar mafic dykes from central India) were compiled in our database (table 9; figure 1). Some intermediate rocks with $\left(\mathrm{SiO}_{2}\right)_{\text {adj }}<53 \%$ were also included to confirm our earlier indications that the inclusion of some, a few additional rocks of intermediate composition does not change the conclusions reached by the use of our discriminant function diagrams.

\subsubsection{Malani igneous suite, northwestern India}

Malani was mainly an acid volcanic/plutonic event $(\sim 750 \mathrm{Ma})$, with rhyolitic and granitic rocks distributed over an area of about $51,000 \mathrm{~km}^{2}$, whose origin has been attributed to a hot spot (Kochhar
1984; Singh and Vallinayagam 2004) or to extensional tectonics and intraplate anorogenic setting (Sharma 2004). Based on tectonomagmatic diagrams for granites, all workers have called the Malani volcanics and granites as 'A-type' or 'anorogenic'. Surprisingly, barring few flows and dolerite dykes, basalts and other basic rocks are rare; the basic and intermediate rocks together constitute less than $5 \%$ of the total area (Bhushan and Chandrasekaran 2002). To our knowledge, no one has yet used the chemical composition of basalts in Malani to infer tectonic setting.

First of all, for rock nomenclature we wish to point out the danger of not strictly following the recommendations of IUGS Subcommission on the Systematics of Igneous Rocks. Unfortunately, this recommendation-violation is a generalized phenomenon. These problems seem to persist in numerous published papers, including those from Malani. For example, Sharma (2004) in his table $6 \mathrm{~A}$ on Malani volcanics reported the first 5 samples as basalt, none of them proved to be a basic rock (basalt) when the SINCLAS computer program (Verma et al 2002) was used. Even the sample listed by him as basalt in his table 6B (data from Maheshwari et al 1996) did not result as a basalt. In fact, all basalt samples from Sharma (2004) are basaltic andesite rocks according to the IUGS recommendations and TAS classification scheme.

We, therefore, strongly recommend geologists to use the SINCLAS computer program for proper rock nomenclature and CIPW norm calculation, as has been already done by numerous workers (e.g., Carracedo et al 2003; Sheth et al 2003; Srivastava 

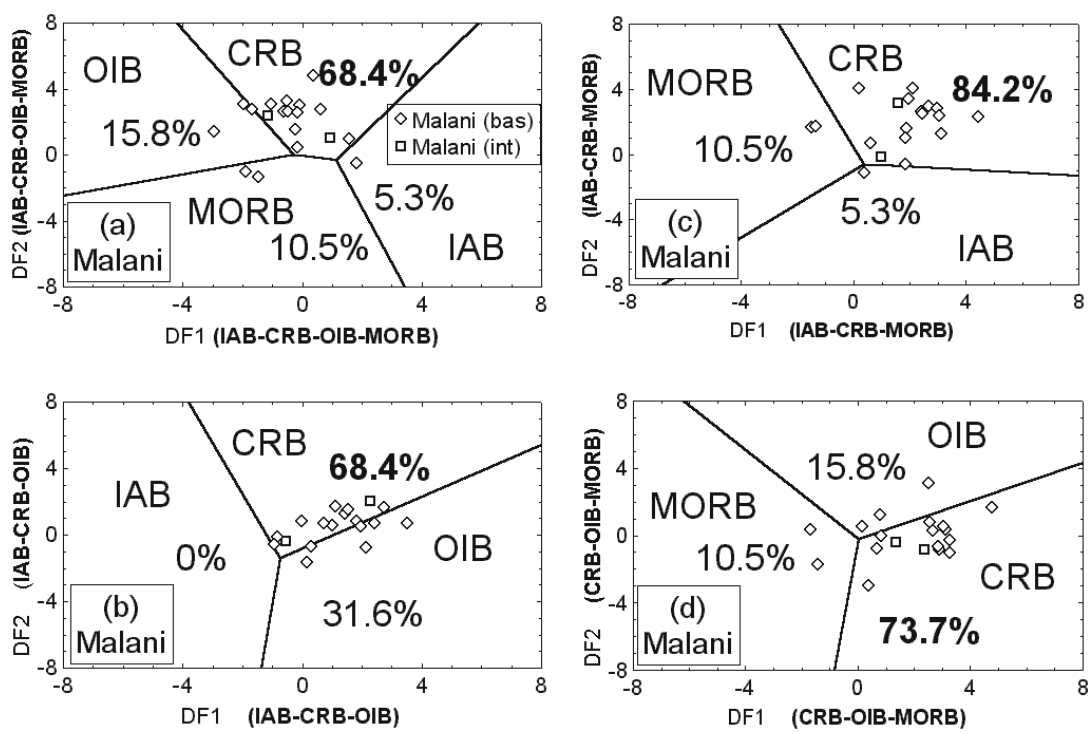

Figure 13. Four pertinent diagrams for samples of basic rocks from a cratonic area of India - the Malani igneous suite, northwestern Indian shield (2 samples of intermediate rocks with $\left(\mathrm{SiO}_{2}\right)_{\text {adj }}<53 \%$ are also included). Note most samples for the Malani area fall in the CRB field; the corresponding \% success rates (68.4 to $84.2 \%)$ are high. The figure parts (a) to (d) are the same as in figure 7 .

et al 2004a, 2004b; Srivastava and Sinha 2004; Singh and Kumar 2005; Treviño-Cázares et al 2005; Rajani et al 2005). This recommendation is also valid for other workers such as: Sensarma et al (2004) on Bijli Rhyolite of central India; Alam et al (2004) on Barren Island lavas and dykes; and Duraiswami et al (2004) on Daund lava flow. We hope that, in future, these authors and others use SINCLAS for their work and especially if they employ our discriminant function diagrams for evaluating the tectonic setting of their study areas.

Unfortunately, high-quality major-element data for basic rocks from Malani are rather scarce; some of these analyses sum to even much less than $97 \%$ (and that is so even after including the reported values of $\mathrm{H}_{2} \mathrm{O}^{+}$and $\mathrm{H}_{2} \mathrm{O}^{-}$; see for example, Bhushan and Chandrasekaran 2002). For other samples the reported concentration of $\mathrm{P}_{2} \mathrm{O}_{5}$ is zero. Thus, at present only 17 samples of basic rocks with an 'acceptable' quality of major-element data could be compiled in our application database, and two samples of intermediate rocks (with $\left.\left(\mathrm{SiO}_{2}\right)_{\text {adj }}<53 \%\right)$ were included to increase this number and to check if these 'nearly basic' magmas provided similar results. The data for the Malani suite are plotted in figure 13(a-d). It appears that the results support the CRB as the probable tectonic setting because about 68.4 to $84.2 \%$ samples fall in this field (figure 13a-d). These \% success rate calculations included the two intermediate rocks also considered in these diagrams. The conclusions for the Malani suite, however, should not be taken as definitive, because the number of samples considered is rather small, but the indications are that a larger number of samples should confirm our preliminary results.

\subsubsection{Bastar dyke complex, central India}

The Precambrian mafic dykes that transect in this area of the Archaean granite gneisses of the southern Bastar craton have been studied by Srivastava et al (1996) and Srivastava and Singh (2003, 2004). The major-element data for 65 samples of basic rocks (mafic dykes) from this area plotted in our discriminant function diagrams (figure 14a-d) indicate the IAB as the more likely tectonic setting. These rocks show for the IAB \% success rates that vary from 59.0 to $67.2 \%$, which are rather low, and therefore, the IAB indications for Bastar should not be taken as definitive. Probably other lines of evidence are needed to confirm these tentative results.

We presented these two examples of Malani and Bastar to stress that representative data from ancient areas, especially for relatively less-altered rocks, are needed to draw statistically meaningful conclusions from discriminant function diagrams. The alteration effects on major-element chemistry may also complicate the results of the discrimination diagrams, which probably is the case of the Bastar area.

\subsection{Final comments and future plans}

Although the utility of these diagrams for ancient (Precambrian) rocks has been demostrated by 

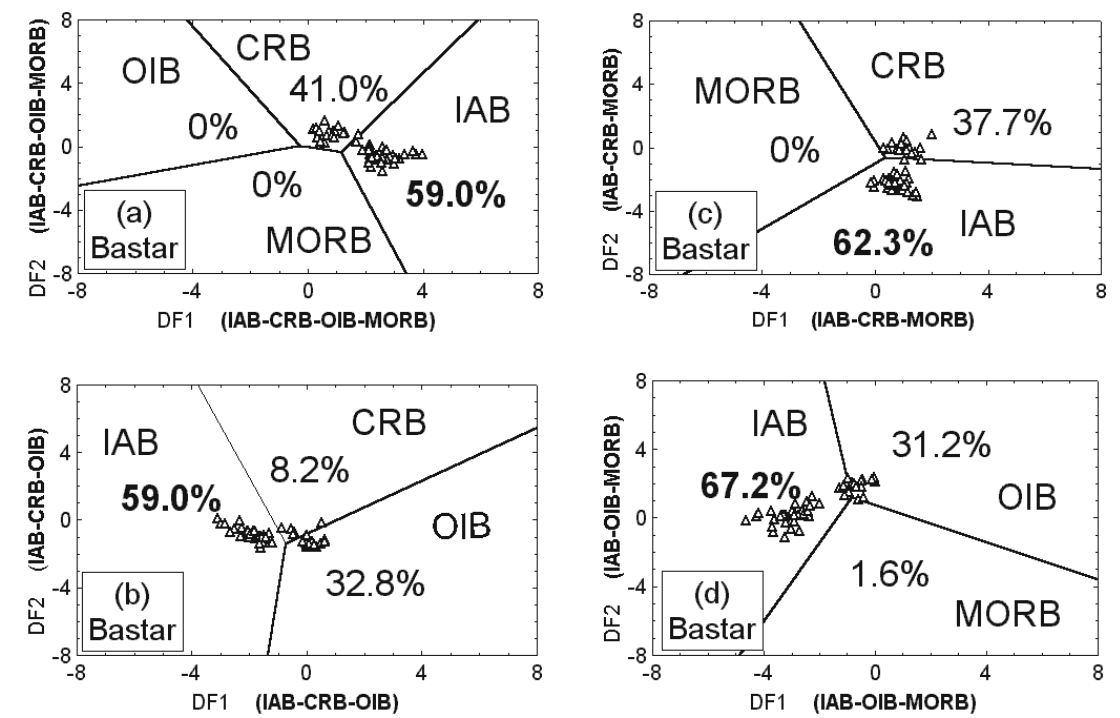

Figure 14. Four pertinent diagrams for samples of basic rocks from a cratonic area of India - the Bastar craton, central India (the Bastar mafic dykes). Note most samples fall in the IAB field although the corresponding \% success rates (59.0 to $67.2 \%$ ) are only moderately high. The figure parts (a) to (c) are the same as in figure 7, but (d) is the three-group IAB, OIB, and MORB (1-3-4).

some selected examples from India, the diagrams may not work well for highly altered and metamorphosed rocks. Their success will depend in a complex manner on the amount of changes reflected in the concentration ratios and the respective discriminant function coefficients. In such situation our suggestion is to use these new diagrams with extreme caution, and accept the results only if independent evidence for the inferred tectonic setting is available for a given area.

Work is currently in progress to extend our statistically-correct methodology of discrimination analysis to include trace elements, especially the so-called immobile elements, which might provide in future additional diagrams with probably a better performance for older areas than the existing ones. Additionally, use of isotopic ratios is also likely to be of great value in building new discriminant function diagrams; the utility of such ratios has already been documented by Verma (2002) for distinguishing the volcanism in southern Mexico from the Central American Volcanic Arc. Ironically, both areas correspond to the same oceanic plate (Cocos plate), and yet the tectonic setting inferred on land varies from the conventional continental arc in central America to an unexpected rift setting in southern Mexico. Similarly, incorporation of additional tectonic settings such as flood basalt province and continental arc as well as division of IAB into main-arc and back-arc (basin) settings and of MORB into normal-MORB and enriched-MORB (islands close to mid-ocean ridges) should also be considered. In the mean time, the utility of our new discriminant function diagrams for complex settings as well as for older terranes has been documented beyond any reasonable doubt.

\section{Conclusions}

The use of discriminant analysis along with prior $\log _{\mathrm{e}}$-transformation of concentration ratios provided a powerful and statistically-correct method for proposing five new diagrams for basic rocks from four tectonic settings. A comparison of the results of the training and testing sets revealed for all cases, consistently high rates of correct classification (overall rates of $84-97 \%$ for both sets). These rates were found to be higher than those obtained by the discriminant analysis of raw concentration data (i.e., without any data transformations). These new diagrams were used to show their usefulness in diverse tectonic classification problems of basic rocks, such as recognition of continental arc setting as similar to the island arc setting and the inference of tectonic setting of complex areas of Mexico as well as of older areas of India (the Deccan and two cratons - the Malani igneous suite and the Bastar dyke complex). An Excel template provided with this paper should facilitate the use of these new discriminant function diagrams.

\section{Acknowledgements}

We thank Alfredo Quiroz-Ruiz for scanning some of the literature application data and converting them to Excel data sheets and for solving the 
computer problems faced during the preparation of high-quality figures for final printing. We are also grateful to two reviewers who opted to remain anonymous and the editor Hetu Sheth for their numerous constructive comments on an earlier version of this paper.

\section{References}

Agrawal S 1999 Geochemical discrimination diagrams: a simple way of replacing eye-fitted boundaries with probability based classifier surfaces; J. Geol. Soc. India 54 335-346.

Agrawal S, Guevara M and Verma S P 2004 Discriminant analysis applied to establish major-element field boundaries for tectonic varieties of basic rocks; Int. Geol. Rev. 46 575-594.

Aitchison J 1986 The statistical analysis of compositional data; (London: Chapman and Hall) 416 p.

Alam M A, Chandrasekharam D, Vaselli O, Capaccioni B, Manetti P and Santo P B 2004 Petrology of the prehistoric lavas and dyke of the Barren island, Andaman Sea, Indian Ocean; Proc. Indian Acad. Sci. (Earth Planet. Sci.) $113715-722$.

Aldanmaz E, Pearce J A, Thirlwall M F and Mitchell J G 2000 Petrogenetic evolution of late Cenozoic, postcollision volcanism in western Anatolia, Turkey; J. Volcanol. Geotherm. Res. 102 67-95.

Alici P, Temel A and Gourgaud A 2002 Pb-Nd-Sr isotope and trace element geochemistry of Quaternary extensionrelated alkaline volcanism: a case study of Kula region (western Anatolia, Turkey); J. Volcanol. Geotherm. Res. 115 487-510.

Aoki K-I, Yoshida T, Yusa K and Nakamura Y 1985 Petrology and geochemistry of the Nyamuragira volcano, Zaire; J. Volcanol. Geotherm. Res. 25 1-28.

Arculus R J 1976 Geology and geochemistry of the alkali basalt-andesite association of Grenada, Lesser Antilles island arc; Geol. Soc. Am. Bull. 87 612-624.

Armstrong-Altrin J S and Verma S P 2005 Critical evaluation of six tectonic setting discrimination diagrams using geochemical data of Neogene sediments from known tectonic settings; Sedim. Geol. 177 115-129.

Auchapt A, Dupuy C, Dostal J and Kanika M 1987 Geochemistry and petrogenesis of rift-related volcanic rocks from South Kivi (Zaire); J. Volcanol. Geotherm. Res. 31 33-46.

Bach W, Hegner E, Erzinger J and Satir M 1994 Chemical and isotopic variations along the superfast spreading East Pacific Rise from 6 to $30^{\circ} \mathrm{S}$; Contrib. Mineral. Petrol. 116 365-380.

Bach W, Erzinger J, Dosso L, Bollinger C, Bougault H, Etoubleau J and Sauerwein J 1996 Unusually large NbTa depletions in North Chile ridge basalts at $36^{\circ} 50^{\prime}$ to $38^{\circ} 56^{\prime} \mathrm{S}$ : major element, trace element, and isotopic data; Earth Planet. Sci. Lett. 142 223-240.

Barberi F, Ferrara G, Santacroce R, Treuil M and Varet J 1975 A transitional basalt-pantellerite sequence of fractional crystallization, the Boina centre (Afar rift, Ethiopia); J. Petrol. 16 22-56.

Bardintzeff J M and Deniel C 1992 Magmatic evolution of Pacaya and Cerro Chiquito volcanological complex, Guatemala; Bull. Volcanol. 54 267-283.

Barrat J A, Joron J L, Taylor R N, Fourcade S, Nesbitt R W and Jahn B M 2003 Geochemistry of basalts from Manda Hararo, Ethiopia: LREE-depleted basalts in Central Afar; Lithos 69 1-13.
Barsdell M 1988 Petrology and petrogenesis of clinopyroxene-rich tholeiitic lavas, Merelava volcano, Vanuatu; J. Petrol. 29 927-964.

Barsdell M and Berry R F 1990 Origin and evolution of primitive island arc ankaramites from western Epi, Vanuatu; J. Petrol. 31 747-777.

Basu A R, Junwen W, Wankang H, Guanghong X and Tatsumoto M 1991 Major element, REE, and $\mathrm{Pb}, \mathrm{Nd}$ and $\mathrm{Sr}$ isotopic geochemistry of Cenozoic volcanic rocks of eastern China: implications for their origin from suboceanic-type mantle reservoirs; Earth Planet. Sci. Lett. 105 149-169.

Bau M and Knittel U 1993 Significance of slab-derived partial melts and aqueous fluids for the genesis of tholeiitic and calc-alkaline island-arc basalts: evidence from Mt. Arayat, Philippines; Chem. Geol. 105 233-251.

Bell K and Peterson T $1991 \mathrm{Nd}$ and Sr isotopic systematics of Shombole volcano, East Africa, and the links between nephelinites, phonolites, and carbonatites; Geology $19582-585$.

Bergmanis E C, Sinton J M and Trusdell F A 2000 Rejuvenated volcanism along the southwest rift zone, East Maui, Hawaii; Bull. Volcanol. 62 239-255.

Bevington P R and Robinson D K 2003 Data reduction and error analysis for the physical sciences (Third Edition); (Boston: McGraw Hill) 320 p.

Bhushan S K and Chandrasekaran V 2002 Geology and geochemistry of the magmatic rocks of the Malani Igneous Suite and Tertiary Alkaline Province of western Rajasthan; Geol. Surv. India Memoir. 126 $1-181$.

Bloomer S H, Stern R J, Fisk E and Geschwind C H 1989 Shoshonitic volcanism in the northern Mariana arc. 1. Mineralogic and major and trace element characteristics; J. Geophys. Res. 94 4469-4496.

Bohrson W A and Reid M R 1995 Petrogenesis of alkaline basalts from Socorro Island, Mexico: Trace element evidence for contamination of ocean island basalt in the shallow ocean crust; J. Petrol. 100 24,555-24,576.

Bondre N R, Hart W K and Sheth H C 2006 Geology and geochemistry of the Sangamner mafic dike swarm, western Deccan volcanic province, India: implications for regional stratigraphy; J. Geol. 114 155-170.

Bougault H, Dmitriev L, Schilling J G, Sobolev A, Joron J L and Needham H D 1988 Mantle heterogeneity from trace elements: MAR triple junction near $14^{\circ} \mathrm{N}$; Earth Planet. Sci. Lett. 88 27-36.

Brophy J G 1986 The Cold Bay volcanic center, Aleutian volcanic arc. I. Implications for the origin of hi-alumina arc basalt; Contrib. Mineral. Petrol. 93 368-380.

Brown G M, Holland J G, Sigurdsson H, Tomblin J F and Arculus R J 1977 Geochemistry of the Lesser Antilles volcanic island arc; Geochim. Cosmochim. Acta $\mathbf{4 1}$ 785-801.

Bryan W B, Stice G D and Ewart A 1972 Geology, petrography, and geochemistry of the volcanic islands of Tonga; J. Geophys. Res. 77 1566-1585.

Bryan W B, Thompson G and Ludden J N 1981 Compositional variation in normal MORB from $22^{\circ}-25^{\circ} \mathrm{N}$ : midAtlantic ridge and Kane fracture zone; J. Geophys. Res. 86 11,815-11,836.

Butler J C 1979 Trends in ternary petrologic variation diagrams - fact or fantasy?; Am. Mineral. 64 1115-1121.

Butler J C 1986 The role of spurious correlation in the development of a komatiite alteration model; J. Geophys. Res. 91 E275-E280.

Butler J C and Woronow A 1986 Discrimination among tectonic settings using trace element abundances of basalts; J. Geophys. Res. 91 10,289-10,300. 
Cambon P, Bougault H, Joron J L and Treuil M 1979 Basalts from the East Pacific Rise: an example of typical oceanic crust depleted in hygromagmaphile elements; In: Init. Repts. DSDP (eds) B T R Lewis and P Robinson et al (Washington DC: US Government Printing Office) 623-633.

Camp V E, Roobol M J and Hooper P R 1991 The Arabian continental alkali basalt province: part II. Evolution of Harrats Khaybar, Ithnayn, and Kura, Kingdom of Saudi Arabia; Geol. Soc. Am. Bull. 103 363-391.

Carr M J 1984 Symmetrical and segmented variation of physical and geochemical characteristics of the Central American volcanic front; J. Volcanol. Geotherm. Res. 20 231-252.

Carr M J, Feigenson M D and Bennett E A 1990 Incompatible element and isotopic evidence for tectonic control of source mixing and melt extraction along the Central American arc; Contrib. Mineral. Petrol. 105 369-380.

Carracedo J C, Singer B, Jicha B, Guillou H, Rodríguez Badiola E, Meco J, Pérez Torrado F J, Gimeno D, Socorro S and Láinez A 2003 La erupción y el tubo volcánico del volcán Corona (Lanzarote, Islas Canarias); Estudios Geol. 59 277-302.

Chauvel C and Jahn B-M 1984 Nd-Sr isotope and REE geochemistry of alkali basalts from the Massif Central, France; Geochim. Cosmochim. Acta 48 93-110.

Chauvel C, McDonough W, Guille G, Maury M and Duncan R 1997 Contrasting old and young volcanism in Rurutu Island, Austral chain; Chem. Geol. 139 125-143.

Chayes F 1960 On correlation between variables of constant sum; J. Geophys. Res. 65 4185-4193.

Chayes F 1978 Ratio correlation. A manual for students of petrology and geochemistry; (Chicago and London: University of Chicago Press) $99 \mathrm{p}$.

Chayes F 1983 Detecting nonrandom associations between proportions by tests of remaining-space variables; Math. Geol. 15 197-206.

Chayes F and Velde D 1965 On distinguishing basaltic lavas of circumoceanic and oceanic-island type by means of discriminant functions; J. Amer. Sci. 263 206-222.

Chen C Y, Frey F A, Garcia M O, Dalrymple G B and Hart S R 1991 The tholeiite to alkalic basalt transition at Haleakala volcano, Maui, Hawaii; Contrib. Mineral. Petrol. 106 183-200.

Chen C-Y, Frey F A and Garcia M O 1990 Evolution of alkalic lavas at Heleakala volcano, east Maui, Hawaii; Contrib. Mineral. Petrol. 105 197-218.

Cheng Q C, Macdougall J D and Lugmair G W 1993 Geochemical studies of Tahiti, Teahitia and Mahetia, Society Island Chain; J. Volcanol. Geotherm. Res. 55 155-184.

Chung S L, Jahn B M, Chen S J, Lee T and Chen C-H 1995 Miocene basalts in northwestern Taiwan: Evidence for EM-type mantle sources in the continental lithosphere; Geochim. Cosmochim. Acta 59 549-555.

Chung S-L, Sun S S, Tu K, Chen-Hong C and Lee C L 1994 Late Cenozoic basaltic volcanism around the Taiwan Strait, SE China: product of lithosphere-asthenosphere interaction during continental extension; Chem. Geol. $1121-20$

Class C, Altherr R, Volker F, Eberz G and McCulloch M T 1994 Geochemistry of Pliocene to Quaternary alkali basalts from the Huri Hills, Northern Kenya; Chem. Geol. $1131-22$

Cullen A and McBirney A R 1987 The volcanic geology and petrology of Isla Pinta, Galapagos archipelago; Geol. Soc. Am. Bull. 98 294-301.

Davidson J P and Wilson I R 1989 Evolution of an alkali basalt-trachyte suite from Jebel Marra volcano, Sudan, through assimilation and fractional crystallization; Earth Planet. Sci. Lett. 95 141-160.

De Mulder M, Hertogen J, Deutsch S and André L 1986 The role of crustal contamination in the potassic suite of the Karisimbi volcano (Virunga, African Rift Valley); Chem. Geol. 57 117-136.

Defant M J, Jacques D, Maury R C, De Boer J and Joron J-L 1989 Geochemistry and tectonic setting of the Luzon arc, Philippines; Geol. Soc. Am. Bull. 101 663-672.

Defant M J, Maury R C, Ripley E M, Feigenson M D and Jacques D 1991 An example of island-arc petrogenesis: goechemistry and petrology of the southern Luzon arc, Philippines; J. Petrol. 32 455-500.

Defant M J, Sherman S, Maury R C, Bellon H, de Boer J, Davidson J and Kepezhinskas P 2001 The geology, petrology, and petrogenesis of Saba Island, Lesser Antilles; J. Volcanol. Geotherm. Res. 107 87-111.

Demant A 1981 L'axe néo-volcanique transmexicain, étude volcanologique et pétrographique, signification géodynamique. Ph.D. thesis, 259 p. and appendix. Université de Droit, d'Economie et des Sciences d'Aix-Marseille.

Deniel C, Vidal P, Coulon C, Vellutini P and Piguet P 1994 Temporal evolution of mantle sources during continental rifting: The volcanism of Djibouti (Afar); J. Geophys. Res. 99 2853-2869.

Deruelle B 1982 Petrology of the Plio-Quaternary volcanism of the south-central and meridional Andes; J. Volcanol. Geotherm. Res. 14 77-124.

Devine J D 1995 Petrogenesis of the basalt-andesite-dacite association of Grenada, Lesser Antilles island arc, revisited; J. Volcanol. Geotherm. Res. 69 1-33.

Dosso L, Bougault H, Beuzart P, J-Y C and Joron J-L 1988 The geochemical structure of the South-East Indian Ridge; Earth Planet. Sci. Lett. 88 47-59.

Dosso L, Bougault H and Joron J-L 1993 Geochemical morphology of the north Mid-Atlantic Ridge, $10^{\circ}-24^{\circ} \mathrm{N}$ : trace element-isotope complementarity; Earth Planet. Sci. Lett. 120 443-462.

Doucet S, Weis D, Scoates J S, Debaille V and Giret A 2004 Geochemical and Hf-Pb-Sr-Nd isotopic constraints on the origin of the Amsterdam-St Paul (Indian Ocean) hotspot basalts; Earth Planet. Sci. Lett. 218 179-195.

Duncker K E, Wolff J A. Harmon R S, Leat P T, Dickin A P and Thompson R N 1991 Diverse mantle and crustal components in lavas of the NW Cerros del Rio volcanic field, Rio Grande Rift, New Mexico; Contrib. Mineral. Petrol. 108 331-345.

Dupuy C, Dostal J, Marcelot G, Bougault H, Joron J L and Treuil M 1982 Geochemistry of basalts from central and southern New Hebrides arc: implication for their source rock composition; Earth Planet. Sci. Lett. 60 207-225.

Dupuy C, Barsczus H G, Liotard J M and Dostal J 1988 Trace element evidence for the origin of ocean island basalts: an example from the Austral Islands (French Polynesia); Contrib. Mineral. Petrol. 98 293-302.

Dupuy C, Barsczus H G, Dostal J, Vidal P and Liotard J-M 1989 Subducted and recycled lithosphere as the mantle source of Ocean Island basalts from southern Polynesia, central Pacific; Chem. Geol. 77 1-18.

Duraiswami R A, Bondre N R and Dole G 2004 Possible lava tube system in a hummocky lava flow at Daund, western Deccan volcanic province, India; Proc. Indian Acad. Sci. (Earth Planet. Sci.) $\mathbf{1 1 3} 819-829$

Edwards C M H, Menzies M A, Thirlwall M F, Morris J D, Leeman W P and Harmon R S 1994 The transition to potassic alkaline volcanism in Island arcs: The RinggitBeser complex, East Java, Indonesia; J. Petrol. 35 $1557-1595$. 
Elliott T, Plank T, Zindler A, White W M and Bourdon B 1997 Element transport from slab to volcanic front at the Mariana arc; J. Geophys. Res. 102 14,991-15,019.

Engel A E J and Engel C G 1964 Igneous rocks of the East Pacific Rise; Science 146 477-485.

Ewart A, Brothers R N and Mateen A 1977 An outline of the geology and geochemistry, and the possible petrogenetic evolution of the volcanic rocks of the Tonga-KermadecNew Zealand island arc; J. Volcanol. Geotherm. Res. 2 205-270.

Fan Q and Hooper P R 1991 The Cenozoic basaltic rocks of eastern China: petrology and chemical composition; J. Petrol. 32 765-810.

Ferrari L, Tagami T, Eguchi M, Orozco-Esquivel M T, Petrone C M, Jacobo-Albarrán J and López-Martínez M 2005 Geology, geochronology and tectonic setting of Late Cenozoic volcanism along the southwestern Gulf of Mexico: the Eastern Alkaline Province revisited; J. Volcanol. Geotherm. Res. 146 284-306.

Ferriz H and Mahood G A 1987 Strong compositional zonation in a silicic magmatic system: Los Humeros, Mexican Neovolcanic Belt; J. Petrol. 28 171-209.

Feuerbach D L, Smith E I, Walker J D and Tangeman J A 1993 The role of the mantle during crustal extension: constraints from geochemistry of volcanic rocks in the Lake Mead area, Nevada and Arizona; Geol. Soc. Am. Bull. 105 1561-1575.

Fitton J G, James D and Leeman W P 1991 Basic magmatism associated with Late Cenozoic extension in the western United States: compositional variations in space and time; J. Geophys. Res. 96 13,693-13,711.

Foden J D and Varne R 1980 The petrology and tectonic setting of Quaternary-Recent volcanic centres of Lombok and Sumbawa, Sunda arc; Chem. Geol. 30 210-226.

Fontaine-Vive M and De Goer De Herve A 1984 Deux exemples de mélange par brassage mécanique entre laves alcalines contrastées dans le strato-volcan du Cantal (Massif Central Francais). Implications volcanologiques; Bull. Volcanol. 47 807-825.

Frey F A, Gerlach D C, Hickey R L, Lopez-Escobar L and Munizaga-Villavicencio F 1984 Petrogenesis of the Laguna del Maule volcanic complex, Chile $\left(36^{\circ} \mathrm{S}\right)$; Contrib. Mineral. Petrol. 88 133-149.

Frey F A, Garcia M O and Roden M F 1994 Geochemical characteristics of Koolau volcano: implications of intershield geochemical differences among Hawaiian volcanoes; Geochim. Cosmochim. Acta 58 1441-1462.

Furman T, Btyce J G, Karson J and Iotti A 2004 East African rift system (EARS) plume structure: insights from Quaternary mafic lavas of Turkana, Kenya; J. Petrol. 45 1069-1088.

Gamble J A, Wright I C, Woodhead J D and McCulloch M T 1995 Arc and back-arc geochemistry in the southern Kermadec arc-Ngatoro basin and offshore Taupo volcanic zone, SW Pacific; In: Volcanism associated with extension at consuming plate margins (ed.) J L Smellie, Geological Society Special Publication 193-212.

Garcia M O, Rhodes J M, Wolfe E W, Ulrich G E and Ho R A 1992 Petrology of lavas from episodes $2-47$ of the Puu Oo eruption of Kilauea Volcano, Hawaii: evaluation of magmatic processes; Bull. Volcanol. 55 1-16.

García-Valladares O, Sánchez-Upton P and Santoyo E 2006 Numerical modelling of flow processes inside geothermal wells: An approach for predicting production characteristics with uncertainties; Ener. Convers. Managem. 47 1621-1643.

Geist D J, McBirney A R and Duncan R A 1986 Geology and petrogenesis of lavas from San Cristobal Islands, Galapos Archipelago; Geol. Soc. Am. Bull. 97 555-566.
Gerlach D C, Frey F A, Moreno-Roa H and Lopez-Escobar L 1988 Recent volcanism in the Puyehue-Cordon Caulle region, southern Andes, Chile $\left(40.5^{\circ} \mathrm{S}\right)$ : petrogenesis of evolved lavas; J. Petrol. 29 333-382.

Gibson S A, Thompson R N, Leat P T, Dickin A P, Morrison M A, Hendry G L and Mitchell J G 1992 Asthenosphere-derived magmatism in the Rio Grande rift, western USA: implications for continental break-up; In: Magmatism and the causes of continental break-up; (eds) Storey B C, Alabaster T and Pankhurst R J, Geological Society Special Publication 61-89.

Gómez-Tuena A, LaGatta A B, Langmuir C H, Goldstein S L, Ortega-Gutiérrez F and Carrasco-Núñez G 2003 Temporal control of subduction magmatism in the eastern Trans-Mexican Volcanic Belt: mantle sources, slab contributions, and crustal contamination; G3 4 10.1029/2003GC000524.

Guevara M, Verma S P, Velasco-Tapia F, Lozano-Santa Cruz L and Girón P 2005 Comparison of linear regression models for quantitative geochemical analysis: Example of x-ray fluorescence spectrometry; Geostand. Geoanal. Res. 29 271-284.

Haase K M, Devey C W, Mertz D F, Stoffers P and Dieter G-S 1996 Geochemistry of lavas from Mohns Ridge, Norwegian-Greenland Sea: implications for melting conditions and magma sources near Jan Mayen; Contrib. Mineral. Petrol. 123 223-237.

Hall L S and Sinton J M 1996 Geochemical diversity of the large lava field on the flank of the East Pacific Rise at $8^{\circ} 17^{\prime} \mathrm{S}$; Earth Planet. Sci. Lett. 142 241-251.

Han B-F, Wang S-G and Kagami H 1999 Trace element and Nd-Sr isotope constraints on origin of the Chifeng flood basalts, North China; Chem. Geol. 155 187-199.

Harpp K S, Fornari D J, Geist D J and Kurz M D 2003 Genovesa submarine ridge: a manifestation of plumeridge interaction in the northern Galápagos islands; $G 3$ 4 10.1029/2003GC000531.

Hart W K, WoldeGabriel G, Walter R C and Mertzman S A 1989 Basaltic volcanism in Ethiopia: constraints on continental rifting and mantle interactions; J. Geophys. Res. 94 7731-7748.

Hazlett R W 1987 Geology of San Cristobal volcanic complex, Nicaragua; J. Volcanol. Geotherm. Res. 33 223-230.

Hegner E and Smith I E M 1992 Isotopic compositions of late Cenozoic volcanics from southeast Papua New Guinea: evidence for multi-component sources in arc and rift environments; Chem. Geol. 97 233-249.

Hekinian R, Francheteau J, Armijo R, Cogné J P, Constantin M, Girardeau J, Hey R, Naar D F and Searle R 1996 Petrology of the Easter microplate region in the South Pacific; J. Volcanol. Geotherm. Res. 72 259-289.

Hekinian R, Stoffers P, Devey C, Ackerman D, Hémond C and O'Connor J 1997 Intraplate versus ridge volcanism on the Pacific-Antarctic Ridge near $37^{\circ} \mathrm{S}-111^{\circ} \mathrm{W} ; \mathrm{J}$. Geophys. Res. 102 12,265-12,286.

Hémond C, Devey C W and Chauvel C 1994 Source compositions and melting processes in the Society and Austral plumes (South Pacific Ocean): element and isotope (Sr, $\mathrm{Nd}, \mathrm{Pb}, \mathrm{Th})$ geochemistry; Chem. Geol. 115 7-45.

Hickey R L, Frey F A, Gerlach D C and Lopez-Escobar L 1986 Multiple sources for basaltic arc rocks from the southern volcanic zone of the Andes $\left(34^{\circ}-41^{\circ} \mathrm{S}\right)$ : trace element and isotopic evidence for contributions from subducted oceanic crust, mantle, and continental crust; J. Geophys. Res. 91 5963-5983.

Hickey-Vargas R, Moreno Roa H, Lopez Escobar L and Frey F A 1989 Geochemical variations in Andean basaltic and silicic lavas from the Villarrica-Lanin volcanic chain 
$\left(39.5^{\circ} \mathrm{S}\right)$ : an evaluation of source heterogeneity, fractional crystallization and crustal assimilation; Contrib. Mineral. Petrol. 103 361-386.

Hole M J, Saunders A D, Marriner G F and Tarney J 1984 Subduction of pelagic sediments: implications for the origin of Ce-Anomalous basalts from the Marianas Islands; J. Geol. Soc. London 141 453-472.

Hoogewerff J A, van Bergen M J, Vroon P Z, Hertogen J, Wordel R, Sneyers A, Nasution A, Varekamp J C, Moens H L E and Mouchel D 1997 U-series, Sr-Nd-Pb isotope and trace-element systematics across an active island arc-continent collision zone: implications for element transfer at the slab-wedge interface; Geochim. Cosmochim. Acta 61 1057-1072.

Hsu C-N, Chen J-C and Ho K-S 2000 Geochemistry of Cenozoic volcanic rocks from Kirin Province, northeast China; Geochem. J. 34 33-58.

Ielsch G, Caroff M, Barsczus H G, Maury R C, Guillou H, Guille G and Cotten J 1998 Géochimie des basalts de I'ile de Ua Huka (archipel des MArquises): vartiation du taux de fusion partielle et hétérogénéité de la source mantellique; C. R. Acad. Sci. Paris 326 413-420.

Ishikawa T, Tera F and Nakazawa T 2001 Boron isotope and trace element systematics of the three volcanic zones in the Kamchatka arc; Geochim. Cosmochim. Acta 65 4523-4537.

Johnson C M and Lipman P W 1988 Origin of metaluminous and alkaline volcanic rocks of the Latir volcanic field, northern Rio Grande rift, New Mexico; Contrib. Mineral. Petrol. 100 107-128.

Kabeto K, Sawada Y, Iizumi S and Wakatsuki T 2001 Mantle sources and magma-crust interactions in volcanic rocks from northern Kenya rift: geochemical evidence; Lithos 56 111-136.

Kay S M and Kay R W 1994 Aleutian magmas in space and time; In: Geology of North America (eds) Plafker G and Berg H C (USA: Geological Society of America) 687-722.

Kay S M, Kay R W and Citron G P 1982 Tectonic controls on tholeiitic and calc-alkaline magmatism in the Aleutian arc; J. Geophys. Res. 87 4051-4072.

Kempton P D, Fitton J G, Hawkesworth C J and Ormerod D S 1991 Isotopic and trace element constraints on the composition and evolution of the lithosphere beneath the Southwestern United States; J. Geophys. Res. 96 13,713-13,735.

Kepezhinskas P, McDermott F, Defant M J, Hochstaedter A, Drummond M S, Hawkesworth C J, Koloskov A, Maury R C and Bellon H 1997 Trace element and $\mathrm{Sr}-\mathrm{Nd}-\mathrm{Pb}$ isotopic constraints on a three-component model of Kamchatka arc petrogenesis; Geochim. Cosmochim. Acta 61 577-600.

Kimura J-I, Manton W I, Sun C-H, Iizumi S, Yoshida T and Stern R J 2002 Chemical diversity of the Ueno basalts, Central Japan: identification of mantle and crustal contributions to arc basalts; J. Petrol. 43 1923-1946.

Kita I, Yamamoto M, Asakawa Y, Nakagawa M, Taguchi S and Hasegawa H 2001 Contemporaneous ascent of within-plate type and island-arc type magmas in the Beppu-Shimabara graben system, Kyushu island, Japan; J. Volcanol. Geotherm. Res. 111 99-109.

Knittel U, Hegner E, Bau M and Satir M 1997 Enrichment processes in the sub-arc mantle: a $\mathrm{Sr}-\mathrm{Nd}-\mathrm{Pb}$ isotopic and REE study of primitive arc basalts from the Philippines; Can. Mineral. 35 327-346.

Kochhar N 1984 Malani igneous suite: hot spot magmatism and cratonisation of the northern part of the Indian shield; J. Geol. Soc. India 25 155-161.

Lassiter J C, Blichert-Toft J and Hauri E H 2003 Isotope and trace element variations in lavas from Raivavae and
Rapa, Cook-Austral islands: constraints on the nature of HIMU- and EM-mantle and the origin of mid-plate volcanism in French Polynesia; Chem. Geol. 202 115-138.

Le Bas M J, Le Maitre R W, Streckeisen A and Zanettin B 1986 A chemical classification of volcanic rocks based on the total alkali-silica diagram; J. Petrol. 27 745-750.

Le Maitre R W 1976 Some problems of the projection of chemical data into mineralogical classifications; Contrib. Mineral. Petrol. 56 181-189.

Le Roex A P and Dick H J B 1981 Petrography and geochemistry of basaltic rocks from the Conrad fracture zone on the America-Antarctica Ridge; Earth Planet. Sci. Lett. 54 117-138.

Le Roex A P and Erlank A J 1982 Quantitative evaluation of fractional crystallization in Bouvet Island lavas; J. Volcanol. Geotherm. Res. 13 309-338.

Le Roex A P, Dick H J B, Gulen L, Reid A M and Erlank A J 1987 Local and regional heterogeneity in MORB from the Mid-Atlantic Ridge between $54.5^{\circ} \mathrm{S}$ and $51^{\circ} \mathrm{S}$ : evidence for geochemical enrichment; Geochim. Cosmochim. Acta 51 541-555.

Le Roex A P, Frey F A and Richardson S H 1996 Petrogenesis of lavas from the AMAR Valley and Narrowgate region of the FAMOUS Valley, $36^{\circ}-37^{\circ} \mathrm{N}$ on the Mid-Atlantic Ridge; Contrib. Mineral. Petrol. 124 167-184.

Le Roex A P, Späth A and Zartman R E 2001 Lithospheric thickness beneath the southern Kenya rift: implications from basalt geochemistry; Contrib. Mineral. Petrol. 142 89-106.

Le Roux P J, Le Roux A P and Schilling J-G 2002a Crystallization process beneath the southern Mid-Atlantic Ridge (40-55 $\left.{ }^{\circ} \mathrm{S}\right)$, evidence for high pressure initiation of crystallization; Contrib. Mineral. Petrol. 142 582-602.

Le Roux P J, Le Roux A P, Schilling J-G, Shimizu N, Perkins W W and Pearce N J G 2002b Mantle heterogeneity beneath the southern Mid-Atlantic Ridge: trace element evidence for contamination of ambient asthenospheric mantle; Earth Planet. Sci. Lett. 203 479-498.

Liotard J M, Barsczus H G, Dupuy C and Dostal J 1986 Geochemistry and origin of basaltic lavas from Marquesas Archipelago, French Polynesia; Contrib. Mineral. Petrol. 92 260-268.

Lipman P W, Rhodes R M and Dalrymple G B 1990 The Ninole Basalt - Implications for the structural evolution of Mauna Loa volcano, Hawaii; Bull. Volcanol. 53 $1-19$.

Liu C-Q, Masuda A and Xie G-H 1992 Isotope and traceelement geochemistry of alkali basalts and associated megacrysts from the Huangyishan volcano, Kuandian, Liaoning, NE China; Chem. Geol. 97 219-231.

Liu C-Q, Masuda A and Xie G-H 1994 Major- and traceelement compositions of Cenozoic basalts in eastern China: petrogenesis and mantle source; Chem. Geol. 114 $19-42$.

Lonsdale P, Blum N and Puchelt H 1992 The RRR triple junction at the southern end of the Pacific-Cocos East Pacific Rise; Earth Planet. Sci. Lett. 109 73-85.

López-Escobar L, Kilian R, Kempton P D and Tagiri M 1993 Petrography and geochemistry of Quaternary rocks from the southern volcanic zone of the Andes between $41^{\circ} 30^{\prime}$ and $46^{\circ} 00^{\prime} \mathrm{S}$, Chile; Rev. Geol. Chile $2033-35$.

Luhr J F, Aranda-Gómez J J and Housh T B 1995 San Quintín volcanic field, Baja California Norte, México: geology, petrology, and geochemistry; J. Geophys. Res. $10010,353-10,380$.

Lum C C L, Leeman W P, Foland K A, Kargel J A and Fitton J G 1989 Isotopic variations in continental basaltic lavas as indicators of mantle heterogeneity: examples 
from the western U.S. Cordillera; J. Geophys. Res. 94 7871-7884.

Maaløe S, James D, Smedley P, Petersen S and Germann L B 1992 The Koloa volcanic suite of Kauai, Hawaii; J. Petrol. 33 761-784.

Macdonald R, Davies G R, Upton B G J, Denkley P N, Smith M and Leat P T 1995 Petrogenesis of Silali volcano, Gregory rift, Kenya; J. Geol. Soc. London 152 703-720.

Macdonald R, Rogers N W, Fitton J G, Black S and Smith M 2001 Plume-lithosphere interactions in the generation of the basalts of the Kenya rift, East Africa; J. Petrol. 42 877-900.

Maheshwari A, Coltorti M, Sial AN and Mariano G 1996 Crustal influences in the petrogenesis of the Malani rhyolite, southwestern Rajasthan: combined trace element and oxygen isotope constraints; J. Geol. Soc. India 47 611-619.

Mahoney J, Le Roex A P, Peng Z, Fisher R L and Natland J H 1992 Southwestern limits of Indian Ocean ridge mantle and the origin of low ${ }^{206} \mathrm{~Pb} /{ }^{204} \mathrm{~Pb}$ MidOcean Ridge Basalt: isotope systematics of the central southwestern Indian Ridge $\left(17^{\circ}-50^{\circ} \mathrm{E}\right) ; \mathrm{J}$. Geophys. Res. 97 19,771-19,790.

McMillan N J, Dickin A P and Haag D 2000 Evolution of magma source regions in the Rio Grande rift, southern New Mexico; Geol. Soc. Am. Bull. 112 1582-1593.

Melluso L, Beccaluva L, Brotzu P, Gregnanin A, Gupta A K, Morbidelli L and Traversa G 1995 Constraints on the mantle sources of the Deccan Traps from the petrology and geochemistry of the basalts of Gujarat state (western India); J. Petrol. 36 1393-1432.

Melluso L, Barbieri M and Beccaluva L 2004 Chemical evolution, petrogenesis, and regional chemical correlations of the flood basalt sequence in the central Deccan Traps, India; Proc. Indian Acad. Sci. (Earth Planet. Sci.) 113 $587-603$

Middlemost E A K 1989 Iron oxidation ratios, norms and the classification of volcanic rocks; Chem. Geol. 77 19-26.

Miklius A, Flower M F J, Huijsmans J P P, Mukasa S B and Castillo P 1991 Geochemistry of lavas from Taal volcano, southwestern Luzon, Philippines: evidence for multiple magma supply systems and mantle source heterogeneity; J. Petrol. 32 593-627.

Monzier M, Robin C, Eissen J-P and Cotten J 1997 Geochemistry vs. seismo-tectonics along the volcanic New Hebrides Central Chain (Southwest Pacific); J. Volcanol. Geotherm. Res. 78 1-29.

Morrison D F 1990 Multivariate statistical methods (Third Edition); (New York: McGraw-Hill) 495 p.

Morton-Bermea O 1990 Zur petrologie des AlkaligesteinsIntrusivkomplexes der Sierra de Picachos (Nuevo León, Mexiko); Diplomarbeit thesis. Universität Karlsruhe, Karlsruhe, Germany, 115 p.

Moyer T C and Esperança S 1989 Geochemical and isotopic variations in a bimodal magma system: the Kaiser Spring volcanic field, Arizona; J. Geophys. Res. 94 7841-8759.

Mullen E D $1983 \mathrm{MnO} / \mathrm{TiO}_{2} / \mathrm{P}_{2} \mathrm{O}_{5}$ : a minor element discrimination for basaltic rocks of oceanic environments and its implications for petrogenesis; Earth Planet. Sci. Lett. 62 53-62.

Myers J D, Marsh B D and Sinha A K 1985 Strontium isotopic and selected trace element variations between two Aleutian volcanic centers (Adak and Atka): implications for the development of arc volcanic plumbing systems; Contrib. Mineral. Petrol. 91 221-234.

Myers J D, Marsh B D, Frost C D and Linton J A 2002 Petrologic constraints on the spatial distribution of crustal magma chambers, Atka volcanic center, central Aleutian arc; Contrib. Mineral. Petrol. 143 567-586.
Nakagawa M, Ishizuka Y, Kudo T, Yoshimoto M, Hirose W, Ishizaki Y, Gouchi N, Katsui Y, Solovyow A W, Steinberg G S and Abdurakhmanov A I 2002 Tyatya volcano, southwestern Kuril arc: recent eruptive activity inferred from widespread tephra; The Island Arc 11 236-254.

Negendank J F W, Emmermann R, Krawczyk R, Mooser F, Tobschall H and Werle D 1985 Geological and geochemical investigations on the eastern Trans Mexican Volcanic Belt; Geofis. Int. 24 477-575.

Nelson S A, Gonzalez-Caver E and Kyser T K 1995 Constraints on the origin of alkaline and calc-alkaline magmas from the Tuxtla Volcanic Field, Veracruz, Mexico; Contrib. Mineral. Petrol. 122 191-211.

Nick K 1988 Mineralogische, Geochemische und Petrographische Untersuchungen in der Sierra de San Carlos (Mexiko); Unpubl. Doctoral thesis, Universitaet (TH) Fridericiana Karlsruhe, Karlsruhe, Germany, 167 p.

Norman M D and García M O 1999 Primitive magmas and source characteristics of the Hawaiian plume: petrology and geochemistry of shield picrites; Earth Planet. Sci. Lett. 168 27-44.

Nye C J and Reid M R 1986 Geochemistry of primary and least fractionated lavas from Okmok volcano, central Aleutians: implications for arc magma genesis; J. Geophys. Res. 91 10,271-10,287.

Ohara Y, Fujioka K, Ishizuka O and Ishii T 2002 Peridotites and volcanics from the Yap arc system: implications for tectonics of the southern Philippine Sea plate; Chem. Geol. 189 35-53.

Orozco-Esquivel M T 1995 Zur Petrologie des Vulkangebietes von Palma-Sola, Mexiko. Ein Beispiel fuer den Uebergang von anorogenem zu orogenem Vulkanismus; Doctoral thesis. Universitaet Karlsruhe, Karlsruhe, Germany, $167 \mathrm{p}$.

Panter K S, Hart S R, Kyle P, Blusztanjn J and Witch T 2000 Geochemistry of late Cenozoic basalts from the Crary mountains: characterization of mantle sources in Marie Byrd land, Antarctica; Chem. Geol. 165 215-241.

Pearce J A and Cann J R 1971 Ophiolite origin investigated by discriminant analysis using Ti, Zr and Y; Earth Planet. Sci. Lett. 12 339-349.

Pearce J A and Cann J R 1973 Tectonic setting of basic volcanic rocks determined using trace element analyses; Earth Planet. Sci. Lett. 19 290-300.

Pearce J A 1976 Statistical analysis of major element patterns in basalts; J. Petrol. 17 15-43.

Peccerillo A, Barberio M R, Yirgu G, Ayalew D, Barbieri M and Wu T W 2003 Relationships between mafic and peralkaline silicic magmatism in continental rift settings: a petrological, geochemical and isotopic study of the Gedemsa volcano, central Ethiopian rift; J. Petrol. 44 2003-2032.

Peng Z C, Zartman R E, Futa K and Chen D G 1986 Pb-, Srand Nd-isotopic systematics and chemical characteristics of Cenozoic basalts, eastern China; Chem. Geol. 59 3-33.

Peng Z X, Mahoney J, Hooper P, Harris C and Beane J 1994 A role for lower continental crust in flood basalt genesis? Isotopic and incompatible study of the lower six formations of the western Deccan traps; Geochim. Cosmochim. Acta 58 267-288.

Perry F V, Baldridge W S and DePaolo D J 1987 Role of asthenosphere and lithosphere in the genesis of Late Cenozoic basaltic rocks from the Rio Grande and adjacent regions of the southwestern United States; J. Geophys. Res. 92 9193-9213.

Perry F V, Baldridge W S, DePaolo D J and Shafiqullah M 1990 Evolution of a magmatic system during continental extension: the mount Taylor volcanic field, New Mexico; J. Geophys. Res. 95 19,327-19,348. 
Price R C, Kennedy A K, Riggs-Sneeringer M and Frey F A 1986 Geochemistry of basalts from the Indian Ocean triple junction: implications for the generation and evolution of Indian Ocean ridge basalts; Earth Planet. Sci. Lett. 78 379-396.

Rajani R P, Banakar V K, Parthiban G, Mudholkar A V and Chodankar A R 2005 Compositional variation of ferromanganese crusts of the Afanasiy-Nikitin seamount, equatorial Indian Ocean; J. Earth Syst. Sci. 114 51-61.

Ramírez Fernández J A 1996 Zur petrogenese des alkalikomplexes der Sierra de Tamaulipas, NE-Mexiko; Doctoral thesis. Albert-Ludwigs-Universitaet, Freiburg, Germany, $316 \mathrm{p}$.

Raos A M and Crawford A J 2004 Basalts from the Afate Island group, central section of the Vanuatu arc, SW Pacific: geochemistry and petrogenesis; J. Volcanol. Geotherm. Res. 134 35-64.

Reyment R A 1987 Multivariate analysis in Geoscience: fads, fallacies and the future; Chemom. Intell. Lab. Sist. 2 79-91.

Reyment R A and Savazzi E 1999 Aspects of multivariate statistical analysis in geology; (Amsterdam: Elsevier) $285 \mathrm{p}$.

Robin C 1976 Présence simultanée de magmatismes de significations tectoniques opposées dans l'Est du Mexique; Bull. Soc. Geol. Fr. 18 1637-1645.

Robin C 1982 Relations volcanologie-magmatologiegéodynamique: application au passage entre volcanismes alcalin et andésitique dans le sud Mexicain (Axe Transmexicain et Province Alcaline Orientale); Annal. Sci. Univ. Clermont-Ferrand II 31503 p.

Robin C and Tournon J 1978 Spatial relations of andesitic and alkaline provinces of Mexico and Central America; Can. J. Earth Sci. 15 1633-1641.

Rollinson H R 1993 Using geochemical data: evaluation, presentation, interpretation; (Essex: Longman Scientific Technical) $344 \mathrm{p}$.

Romick J D, Perfit M R, Swanson S E and Shuster R D 1990 Magmatism in the eastern Aleutian arc: temporal characteristic of igneous activity on Akutan Island; Contrib. Mineral. Petrol. 104 700-721.

Sakuyama M and Nesbitt R W 1986 Geochemistry of the Quaternary volcanic rocks of the Northeast Japan arc; J. Volcanol. Geotherm. Res. 29 413-450.

Santoyo E, Guevara M and Verma S P 2006 Determination of lanthanides in international geochemical reference materials by reversed-phase high performance liquid chromatography: An application of error propagation theory to estimate total analysis uncertainties; J. Chromatogr. $A$ (in press).

Schilling J-G, Kingsley R H and Devine J D 1982 Galapagos hot spot-spreading center system. 1. Spatial petrological and geochemical variations $\left(83^{\circ} \mathrm{W}-101^{\circ} \mathrm{W}\right) ;$ J. Geophys. Res. 87 5593-5610.

Schilling J-G, Zajac M, Evans R, Johnston T, White W, Devine J D and Kingsley R 1983 Petrologic and geochemical variations along the Mid-Atlantic Ridge from $29^{\circ} \mathrm{N}$ to $73^{\circ} \mathrm{N} ;$ J. Am. Sci. 283 510-586.

Sensarma S, Hoernes S and Mukhopadhyay D 2004 Relative contributions of crust and mantle to the origin of the Bijli rhyolite in a palaeoproterozoic bimodal volcanic sequence (Dongargarh Group), central India; Proc. Indian Acad. Sci. (Earth Planet. Sci.) 113 619-648.

Sharma K K 2004 The Neoproterozoic Malani magmatism of the northwestern Indian shield: implications for crustbuilding processes; Proc. Indian Acad. Sci. (Earth Planet. Sci.) 113 795-807.

Sheth H C, Torres-Alvarado I S and Verma S P 2000 Beyond subduction and plumes: a unified tectonic-petrogenetic model for the Mexican Volcanic Belt; Int. Geol. Rev. 42 1116-1132.

Sheth H C, Torres-Alvarado I S and Verma S P 2002 What is the "calc-alkaline rock series"?; Int. Geol. Rev. 44 686-701.

Sheth H C, Mahoney J J, Baxter A N 2003 Geochemistry of lavas from Mauritius, Indian Ocean: Mande source's and petrogenesis; Int. Geol. Rev. 45 780-797.

Sheth H C, Mahoney J J and Chandrasekharam D 2004 Geochemical stratigraphy of Deccan flood basalts of the Bijasan Ghat section, Satpura range, India; J. Asian Earth Sci. 23 127-139.

Shimizu N and Arculus R J 1975 Rare earth element concentrations in a suite of basanitoids and alkali olivine basalts from Grenada, Lesser Antilles; Contrib. Mineral. Petrol. 50 231-240.

Shinjo R 1998 Petrochemistry and tectonic significance of the emerged late Cenozoic basalts behind the Okinawa Trough Ryukyu arc system; J. Volcanol. Geotherm. Res. $8039-53$.

Shinjo R, Woodhead J D and Hergt J M 2000 Geochemical variation within the northern Ryukyu: magma source compositions and geodynamic implications; Contrib. Mineral. Petrol. 140 263-282.

Siebert L and Carrasco-Núñez G 2002 Late-Pleistocene to precolumbian behind-the-arc mafic volcanism in the eastern Mexican Volcanic Belt; implications for future hazards; J. Volcanol. Geotherm. Res. 115 179-205.

Sims K W W, Blichert-Toft J, Fornari D J, Perfit M R, Goldstein S J, Johnson P, DePaolo D J, Hart S R, Murrell M T, Michael P J, Layne G D and Ball L A 2003 Aberrant youth: chemical and isotopic constraints on the origin of off-axis lavas from the East Pacific Rise, $9^{\circ}-10^{\circ} \mathrm{N}$; G3 4 10.1029/2002GC000443.

Singer B S and Kudo A M 1986 Assimilation-fractional crystallization of Polvadera Group rocks in the northwestern Jemez volcanic field, New Mexico; Contrib. Mineral. Petrol. 94 374-386.

Singer B S, Myers J D and Frost C D 1992a Mid-Pleistocene lavas from the Seguam volcanic center, central Aleutian arc: closed-system fractional crystallization of a basalt to rhyodacite eruptive suite; Contrib. Mineral. Petrol. 110 87-112.

Singer B S, Myers J D and Frost C D 1992b Mid-Pleistocene basalt from the Seguam volcanic center, central Aleutian arc, Alaska: local lithospheric structures and source variability in the Aleutian arc; J. Geophys. Res. 97 4561-4578.

Singh B and Kumar S 2005 Petrogenetic appraisal of early Palaeozoic granitoids of Kinnaur district, higher Himachal Himalaya, India: Gondwana Res. 8 $67-76$.

Singh K A and Vallinayagam G 2004 Geochemistry and petrogenesis of anorogenic basic volcanic-plutonic rocks of the Kundal area, Malani ifneous suite, western Rajasthan, India; Proc. Indian Acad. Sci. (Earth Planet. Sci.) 113 667-681.

Skala W 1977 A mathematical model to investigative distortions of correlation coefficients in closed arrays; Math. Geol. 9 519-528.

Skala W 1979 Some aspects of the constant-sum problem in geochemistry; Chem. Geol. 27 1-9.

Slater L, Jull M, McKenzie D and Gronvöld K 1998 Deglaciation effects on mantle melting under Iceland: results from the northern volcanic zone; Earth Planet. Sci. Lett. 164 151-164.

Smellie J L 1983 A geochemical overview of subductionrelated igneous activity in the South Shetland islands, 
Lesser Antarctica; In: Antarc. Earth Sci. (eds) Oliver R L, James P R and Jago J B (Australian Academy of Sciences and Cambridge University Press) 352-356.

Smith E I, Sánchez A, Walker J D and Wang K 1999 Geochemistry of mafic magmas in the Hurricane Volcanic Field, Utah: implications of small- and large-scale chemical variability of the lithospheric mantle; J. Geol. 107 433-448.

Smith I E M, Stewart R B and Price R C 2003 The petrology of a large intra-oceanic silicic eruption: the Sandy Bay tephra, Kermadec arc, southwest Pacific; J. Volcanol. Geotherm. Res. 124 173-194.

Smith T E, Thirlwall M F and MacPherson C 1996 Trace element and isotope geochemistry of the volcanic rocks of Bequia, Grenadine Islands, Lesser Antilles Arc: a study of subduction enrichment and intra-crustal contamination; J. Petrol. 37 117-143.

Srivastava R K, Hall R P, Verma R and Singh R K 1996 Contrasting Precambrian mafic dykes of the Bastar Craton, Central India: petrological and geochemical characteristics; J. Geol. Soc. India 48 537-546.

Srivastava R K, Chandra R and Shastry A 2004a HighTi type N-MORB parentage of basalts from the south Andaman ophiolite suite, India; Proc. Indian Acad. Sci. (Earth Planet. Sci.) 113 605-618.

Srivastava R K, Singh R K and Verma S P 2004b Neoarchaean mafic volcanic rocks from the southern Bastar greenstone belt, Central India: petrological and tectonic significance; Precamb. Res. 131 305-322.

Srivastava R K and Singh R K 2003 Geochemistry of high$\mathrm{Mg}$ mafic dykes from the Bastar craton: evidence of late Archean boninite-like rocks in an intracratonic setting; Curr. Sci. 85 808-812.

Srivastava R K and Singh R K 2004 Trace element geochemistry and genesis of Precambrian sub-alkaline mafic dikes from the central Indian craton: evidence for mantle metasomatism; J. Asian Earth Sci. 23 373-389.

Srivastava R K and Sinha A K 2004 Geochemistry and petrogenesis of early Cretaceous sub-alkaline mafic dykes from Swangkre-Rongmil, East Garo Hills, Shillong plateau, northeast India; Proc. Indian Acad. Sci. (Earth Planet. Sci.) 113 683-697.

Stephenson D and Marshall T R 1984 The petrology and mineralogy of Mt. Popa volcano and the nature of the late-Cenozoic Burma volcanic arc; J. Geol. Soc. London 141 747-762.

Stolz A J, Varne R, Wheller G E, Foden J D and Abbott M J 1988 The geochemistry and petrogenesis of K-rich alkaline volcanics from the Batu Tara volcano, eastern Sunda arc; Contrib. Mineral. Petrol. 98 374-389.

Stolz A J, Varne R, Davies G R, Wheller G E and Fodon J D 1990 Magma source components in an arc-continent collision zone: the Flores-Lembata sector, Sunda arc, Indonesia; Contrib. Mineral. Petrol. 105 585-601.

Storey M, Rogers G, Saunders A D and Terrell D J 1989 San Quintín volcanic field, Baja California, Mexico: 'within plate' magmatism following ridge subduction; Terra Nova 1 195-202.

Tamura Y 1994 Genesis of island arc magmas by mantle derived bimodal magmatism: evidence from the Shiraham group, Japan; J. Petrol. 35 619-645.

Tatsumi Y, Murasaki M, Arsadi E M and Nohda S 1991 Geochemistry of Quaternary lavas from NE Sulawesi: transfer of subduction components into the mantle wedge; Contrib. Mineral. Petrol. 107 137-149.

Tatsumi Y, Murasaki M and Nohda S 1992 Across-arc variation of lava chemistry in the Izu-Bonin arc: identification of subduction components; J. Volcanol. Geotherm. Res. 49 179-190.
Taylor R N and Nesbitt R W 1998 Isotopic characteristics of subduction fluids in an intra-oceanic setting, Izu-Bonin Arc, Japan; Earth Planet. Sci. Lett. 164 79-98.

Thirlwall M F and Graham A M 1984 Evolution of high-Ca, high-Sr C-series basalts from Grenada, Lesser Antilles: the effects of intra-crustal contamination; J. Geol. Soc. London 141 427-445.

Thirlwall M F, Graham A M, Arculus R J, Harmon R S and Macpherson C G 1997 Resolution of the effects of crustal assimilation, sediment subduction, and fluid transport in island arc magmas: $\mathrm{Pb}-\mathrm{Sr}-\mathrm{Nd}-\mathrm{O}$ isotope geochemistry of Grenada, Lesser Antilles; Geochim. Cosmochim. Acta 60 4785-4810.

Thorpe R S 1977 Tectonic significance of alkaline volcanism in eastern Mexico; Tectonophysics 40 19-26.

Togashi S, Tanaka T, Yoshida T, Ishikawa K-I, Fujinawa A and Kurasawa H 1992 Trace elements and Nd-Sr isotopes of island arc tholeiites from frontal arc of northeast Japan; Geochem. J. 26 261-277.

Tormey D R, Hickey-Vargas R, Frey F A and López-Escobar L 1991 Recent lavas from Andean volcanic front (33 to $42^{\circ} \mathrm{S}$ ): Interpretations of along-arc compositional variations; In: Andean magmatism and its tectonic setting (eds) Harmon R S and Rapela C W, Geological Society of America Special Paper 265 57-77.

Treviño-Cázares A, Ramírez-Fernández J A, Velasco-Tapia F, Rodríguez-Saavedra P 2005 Mantle xenoliths and their host magmas in the Eastern Alkaline Province, northeast Mexico; Int. Geol. Rev. 47 1260-1286.

Trua T, Deniel C and Mazzuoli R 1999 Crustal control in the genesis of Plio-Quaternary bimodal magmatism of the Main Ethiopian Rift (MER): geochemical and isotopic ( $\mathrm{Sr}, \mathrm{Nd}, \mathrm{Pb}$ ) evidence; Chem. Geol. 155 201-231.

Turner S and Foden J 2001 U, Th and Ra disequilibria, $\mathrm{Sr}, \mathrm{Nd}$ and $\mathrm{Pb}$ isotope and trace element variations in Sunda arc lavas: predominance of a subducted sediment component; Contrib. Mineral. Petrol. 142 $43-57$.

Turner S, Foden J, George R, Evans P, Varne R, Elburg $\mathrm{M}$ and Jenner G 2003 Rates and processes of potassic magma evolution beneath Sangeang Api volcano, East Sunda arc, Indonesia; J. Petrol. 44 491-515.

Velasco-Tapia F and Verma S P 2001 First partial melting inversion model for a rift-related origin of the Sierra de Chichinautzin volcanic field, central Mexican Volcanic Belt; Int. Geol. Rev. 43 788-817.

Verma S P 1983 Magma genesis and chamber processes at Los Humeros caldera, Mexico - Nd and Sr isotope data; Nature 301 52-55.

Verma S P 2000a Geochemistry of the subducting Cocos plate and the origin of subduction - unrelated mafic volcanism at the volcanic front of the central Mexican Volcanic Belt; In: Cenozoic tectonics and volcanism of Mexico (eds) Delgado-Granados H, Aguirre-Díaz G and Stock J M, Geological Soceity of America Special Paper 334 195-222.

Verma S P 2000b Error propagation in equations for geochemical modelling of radiogenic isotopes in twocomponent mixing; Proc. Indian Acad. Sci. (Earth Planet. Sci.) 109 79-88.

Verma S P 2000c Geochemical evidence for a lithospheric source for magmas from Los Humeros caldera, Puebla, Mexico; Chem. Geol. 164 35-60.

Verma S P 2001 Geochemical evidence for a lithospheric source for magmas from Acoculco caldera, eastern Mexican Volcanic Belt; Int. Geol. Rev. 43 31-51.

Verma S P 2002 Absence of Cocos plate subduction-related basic volcanism in southern Mexico: a unique case on Earth?; Geology 30 1095-1098. 
Verma S P 2004 Solely extension-related origin of the eastern to west-central Mexican Volcanic Belt (Mexico) from partial melting inversion model; Curr. Sci. 86 $713-719$

Verma S P 2005 Estadística básica para el manejo de datos experimentales: aplicación en la Geoquímica (Geoquimiometría); México, D.F., UNAM, $186 \mathrm{p}$.

Verma S P 2006 Extension related origin of magmas from a garnet-bearing source in the Los Tuxtlas volcanic field, Mexico; Int. J. Earth Sci. (in press).

Verma S P and Schilling J-G 1982 Galapagos hot spot spreading center system. $2 .{ }^{87} \mathrm{Sr} /{ }^{86} \mathrm{Sr}$ and large lithophile element variations $\left(85^{\circ} \mathrm{W}-101^{\circ} \mathrm{W}\right) ; J$. Geophys. Res. 87 $10,838-10,856$.

Verma S P and Santoyo E 1997 New improved equations for $\mathrm{Na} / \mathrm{K}, \mathrm{Na} / \mathrm{Li}$ and $\mathrm{SiO}_{2}$ geothermometers by outlier detection and rejection; J. Volcanol. Geotherm. Res. 79 9-23.

Verma S P, Salazar-V A, Negendank J F W, Milán M, Navarro-L I and Besch T 1993 Características petrográficas y geoquímicas de elementos mayores del campo volcánico de Los Tuxtlas, Veracruz, México; Geofís. Int. 32 237-248.

Verma S P, Torres-Alvarado I S and Sotelo-Rodríguez Z T 2002 SINCLAS: standard igneous norm and volcanic rock classification system; Comput. Geosci. 28 $711-715$.

Verma S P, Torres-Alvarado I S and Velasco-Tapia F 2003 A revised CIPW norm; Schweiz. Miner. Petrog. Mitteil. 83 197-216.

Verma S P, Andaverde J and Santoyo E 2006 Statistical evaluation of methods for the calculation of static formation temperatures in geothermal and oil wells using an extension of the error propagation theory; J. Geochem. Explor. 89 398-404.

Vermeesch P 2006 Tectonic discrimination of basalts with classification trees; Geochim. Cosmochim. Acta $\mathbf{7 0}$ $1839-1848$.

Verwoerd W J, Erlank A J and Kable E J D 1976 Geology and geochemistry of Bouvet island; Proceedings of the Symposium on "Andean and Antarctic Volcanology Problems", Santiago, Chile 201-237.

Walker J A, Carr M J, Feigenson M D and Kalamarides R I 1990 The petrogenetic significance of interstratified high- and low-Ti basalts in central Nicaragua; J. Petrol. 31 1141-1164.

Walker J A, Patino L C, Carr M J and Feigenson M D 2001 Slab control over HFSE depletions in central Nicaragua; Earth Planet. Sci. Lett. 192 533-543.
West H B, Garcia M O, Gerlach D C and Romero J 1992 Geochemistry of tholeiites from Lanai, Hawaii; Contrib. Mineral. Petrol. 112 520-542.

Wheller G E, Varne R, Foden J D and Abbott M J 1987 Geochemistry of Quaternary volcanism in the Sunda-Banda arc, Indonesia, and three-component genesis of islandarc basaltic magmas; J. Volcanol. Geotherm. Res. 32 137-160.

White W M, McBirney A R and Duncan R A 1993 Petrology and geochemistry of the Galápagos Islands: portrait of a pathological mantle plume; J. Geophys. Res. 98 19,533-19,563.

Whitford D J, Nicholls I A and Taylor S R 1979 Spatial variations in the geochemistry of Quaternary lavas across the Sunda arc in Java and Bali; Contrib. Mineral. Petrol. 70 341-356.

Woodhead J D 1988 The origin of geochemical variations in Mariana lavas: a general model for petrogenesis in intraoceanic island arcs; J. Petrol. 29 805-830.

Woodhead J D and Johnson R W 1993 Isotopic and traceelement profiles across the New Britain island arc, Papua New Guinea; Contrib. Mineral. Petrol. 113 479-491.

Woronow A and Love K M 1990 Quantifying and testing differences among means of compositional data; Math. Geol. 22 837-852.

Yellur D D and Nair R S 1978 Assigning a magmatically defined tectonic environment to Chitradurga metabasalts, India, by geochemical methods; Precamb. Res. 7 259-281.

Zellmer G F, Hawkesworth C J, Sparks R S J, Thomas L E, Harford C L, Brewer T S and Loughlin S C 2003 Geochemical evolution of the Soufrière Hills volcano, Montserrat, Lesser Antilles volcanic arc; J. Petrol. 44 1349-1374.

Zhang M, Suddaby P, Thompson R N, Thirlwall M F and Menzies M A 1995 Potassic volcanic rocks in NE China: geochemical constraints on mantle source and magma genesis; J. Petrol. 36 1275-1303.

Zhi X, Song Y, Frey F A, Feng J and Zhai M 1990 Geochemistry of Hannuoba basalts, eastern China: constraints on the origin of continental alkalic and tholeiitic basalt; Chem. Geol. 88 1-33.

Zhuravlev D Z, Tsvetkov A A, Zhuravlev A Z, Gladkov N $\mathrm{G}$ and Chernysheva I V $1987{ }^{143} \mathrm{Nd} /{ }^{144} \mathrm{Nd}$ and ${ }^{87} \mathrm{Sr} /{ }^{86} \mathrm{Sr}$ ratios in recent magmatic rocks of the Kurile Island Arc; Chem. Geol. 66 227-243.

Zou H, Zindler A, Xisheng X and Qi Q 2000 Major, trace element, and $\mathrm{Nd}, \mathrm{Sr}$ and $\mathrm{Pb}$ isotope studies of Cenozoic basalts in SE China: mantle sources, regional variations and tectonic significance; Chem. Geol. 171 33-47. 